TRANSACTIONS OF THE

AMERICAN MATHEMATICAL SOCIETY

Volume 361, Number 12, December 2009, Pages 6613-6644

S 0002-9947(09)04824-7

Article electronically published on July 22, 2009

\title{
IWASAWA DECOMPOSITIONS OF SOME INFINITE-DIMENSIONAL LIE GROUPS
}

\author{
DANIEL BELTIŢĂ
}

\begin{abstract}
We set up an abstract framework that allows the investigation of Iwasawa decompositions for involutive infinite-dimensional Lie groups modeled on Banach spaces. This provides a method to construct Iwasawa decompositions for classical real or complex Banach-Lie groups associated with the Schatten ideals $\mathfrak{S}_{p}(\mathcal{H})$ on a complex separable Hilbert space $\mathcal{H}$ if $1<p<\infty$.
\end{abstract}

\section{Contents}

1. Introduction 6613

2. Iwasawa decompositions for involutive Banach-Lie groups 6616

3. Classical Banach-Lie groups and their Lie algebras $\quad 6623$

4. Iwasawa decompositions for groups of type A 6627

5. Iwasawa decompositions for groups of type B 6634

6. Iwasawa decompositions for groups of type C 6637

7. Decompositions lifted to covering groups 6640

Appendix A. Auxiliary facts on operator ideals 6641

\begin{tabular}{ll} 
Acknowledgment & 6642 \\
\hline
\end{tabular}

References 66642

\section{INTRODUCTION}

Our aim in this paper is to set up an abstract framework that allows us to investigate Iwasawa decompositions for involutive infinite-dimensional Lie groups modeled on Banach spaces. In particular we address an old conjecture on the existence of such decompositions for the classical Banach-Lie groups of operators associated with the Schatten operator ideals on Hilbert spaces (see subsection 8.4 of Section II.8 in Ha72 and Section 3 below), and we show that the corresponding question can be answered in the affirmative in many cases, even in the case of the

Received by the editors February 11, 2008.

2000 Mathematics Subject Classification. Primary 22E65; Secondary 22E46, 47B10, 47L20, $58 \mathrm{~B} 25$.

Key words and phrases. Classical Lie group; Iwasawa decomposition; operator ideal; triangular integral.

(C)2009 American Mathematical Society

Reverts to public domain 28 years from publication 
covering groups (Corollary 7.2 below). To place these results in a proper perspective, we mention that part of our motivation comes from the problem of describing an appropriate class of infinite-dimensional reductive Lie groups, as discussed in the paper $\mathrm{Be} 09]$.

The Iwasawa decompositions of finite-dimensional reductive Lie groups (see, e.g., Iw49, [He01, and [Kn96]) play a crucial role in areas such as differential geometry and representation theory. So far as differential geometry is concerned, there exists a recent growth of interest in group decompositions and their implications in various geometric problems in infinite dimensions; see, for instance, Tu05, Tu06, and the references therein. From this point of view it is natural to try to understand the infinite-dimensional versions of Iwasawa decompositions as well; this problem was already addressed in the case of loop groups in [Ke04 and BD01. As regards the representation theory, it is well known that decompositions of this kind are particularly important for instance in the construction of principal series representations, and there has been a continuous endeavor to extend the ideas of representation theory to the setting of infinite-dimensional Lie groups. Some references related in spirit to the present paper are [Se57, Ki73, SV75, O178 Bo80, Ca85, Ol88, Pic90, Bo93, Nee98, [NØ98, [NRW01, DPW02, Nee04, Gru05, Wo05, BR07]; however, this list is very far from being complete. In this connection we wish to highlight the paper Wo05 devoted to an investigation of direct limits of (Iwasawa decompositions and) principal series representations of reductive Lie groups. In some sense, the results of the present paper can be thought of as belonging to the same line of investigation, inasmuch as the construction of Iwasawa decompositions should be the very first step toward the construction of principal series representations for the classical Banach-Lie groups and their covering groups.

Another source of interest in obtaining Iwasawa decompositions for infinitedimensional versions of reductive Lie groups (cf. [Be09]) is related to the place held by reductive structures in the geometry of many infinite-dimensional manifolds; see, for instance, CG99] and [Nee02b]. We refer also to the recent survey Ga06, which skillfully highlights the special relationship between the reductive structures and the idea of amenability. That relationship also plays an important role in the abstract framework constructed in Section 2 of the present paper. It is noteworthy that reductive structures with a Lie-theoretic flavor constitute the background of the papers [Neu99] and [Neu02] as well, concerning convexity theorems (cf. [Ko73, [LR91, [BFR93]) in an infinite-dimensional setting.

The methods we use to set up the aforementioned abstract framework are largely inspired by the interaction between the theory of Lie algebras and the local spectral theory of bounded operators (see [BS01]). These methods turn out to be particularly effective in order to identify what the third component of an infinitedimensional Iwasawa decomposition should be, that is, the infinite-dimensional versions of nilpotent Lie groups and algebras.

On the other hand, the applications we make to the classical Banach-Lie groups are naturally related to the theory of triangular integrals (GK70, EL72], Er78, [Ara78, Da88]) and to the theory of factorization of Hilbert space operators along nests of subspaces ( Arv67, GK70], Er72], Lar85], Po86], Pit88, [MSS88]). For the reader's convenience we recorded in Appendix A some auxiliary facts on operator ideals, in particular the factorization results suitable for our purposes. We refer to the paper [Be09] for more details. 
The structure of the present paper is outlined in the table of contents at the very beginning. Thus, we are going to set up an abstract framework in Section 2, and then in Section 3 we shall recall the classical Banach-Lie groups under a slightly more general form than usual (see Ha72). By using the general methods developed in Section 2, we shall investigate in Sections 4 6 the existence of Iwasawa decompositions for the three types of classical groups. Finally, Section 7 contains our main result on such decompositions for all the covering groups of the connected 1-components of classical Banach-Lie groups; see Corollary 7.2

Rough decompositions. Before proceeding with the main part of our investigation which leads to the aforementioned corollary, we wish to show a sample of pathological phenomena one has to avoid in order to obtain smooth Iwasawa decompositions for infinite-dimensional Lie groups.

In the next statement and throughout the paper, by an orthonormal basis in a real or complex Hilbert space we mean a complete orthonormal subset.

Proposition 1.1. Let $\mathcal{H}$ be a complex separable Hilbert space with an orthonormal basis $\left\{\xi_{j}\right\}_{0 \leq j<\omega}$, where $\omega \in \mathbb{N} \cup\left\{\aleph_{0}\right\}$. Consider the Banach-Lie group $G:=\operatorname{GL}(\mathcal{H})$ consisting of all invertible bounded linear operators on $\mathcal{H}$, and its subgroups

$$
\begin{aligned}
K & :=\left\{k \in G \mid k^{*} k=\mathbf{1}\right\}, \\
A & :=\left\{a \in G \mid a \xi_{j} \in \mathbb{R}_{+}^{*} \xi_{j} \text { whenever } 0 \leq j<\omega\right\}, \text { and } \\
N & :=\left\{n \in G \mid n \xi_{j} \in \xi_{j}+\operatorname{span}\left\{\xi_{l} \mid l<j\right\} \text { whenever } 0 \leq j<\omega\right\} .
\end{aligned}
$$

In addition, consider the Banach-Lie algebra $\mathfrak{g}=\mathcal{B}(\mathcal{H})$, with its closed Lie subalgebras

$$
\begin{aligned}
\mathfrak{k} & :=\left\{X \in \mathfrak{g} \mid X^{*}=-X\right\}, \\
\mathfrak{a} & :=\left\{Y \in \mathfrak{g} \mid Y \xi_{j} \in \mathbb{R} \xi_{j} \text { whenever } 0 \leq j<\omega\right\}, \text { and } \\
\mathfrak{n} & :=\left\{Z \in \mathfrak{g} \mid Z \xi_{j} \in \operatorname{span}\left\{\xi_{l} \mid l<j\right\} \text { whenever } 0 \leq j<\omega\right\} .
\end{aligned}
$$

Then the following assertions hold:

- $K, A$, and $N$ are Banach-Lie groups with the corresponding Lie algebras $\mathfrak{k}, \mathfrak{a}$, and $\mathfrak{n}$, respectively, and the multiplication map $\boldsymbol{m}: K \times A \times N \rightarrow G$, $(k, a, n) \mapsto k a n$, is smooth and bijective.

- The mapping $\boldsymbol{m}$ is a diffeomorphism if and only if $\mathfrak{k} \dot{+} \mathfrak{a} \dot{+} \mathfrak{n}=\mathfrak{g}$, and this equality holds if and only if the Hilbert space $\mathcal{H}$ is finite-dimensional.

Proof. It is straightforward to prove that $\mathbf{m}$ is injective since $K \cap A N=\{\mathbf{1}\}$. To prove that $\mathbf{m}$ is surjective as well, we can use the unital Banach algebra

$$
\mathfrak{B}=\left\{b \in \mathcal{B}(\mathcal{H}) \mid b \xi_{j} \in \operatorname{span}\left\{\xi_{l} \mid 0 \leq l \leq j\right\} \text { if } 0 \leq j<\omega\right\} .
$$

Denote by $\mathfrak{B}^{\times}$the group of invertible elements in $\mathfrak{B}$. It was proved in Arv75 and Lar85. that for every $g \in \mathrm{GL}(\mathcal{H})$ there exist $k \in K$ and $b \in \mathfrak{B}^{\times}$such that $g=k b$. It is easy to see that $\mathfrak{B}^{\times} \subseteq K A N$; hence $g=k b \in K A N=\mathbf{m}(K \times A \times N)$. The fact that $K, A, N$ are Banach-Lie groups with the corresponding Lie algebras $\mathfrak{k}, \mathfrak{a}$, and $\mathfrak{n}$, respectively, follows for instance by Corollary 3.7 in [Be06], and in addition the inclusion maps of $K, A$, and $N$ into $G$ are smooth. It then follows that the multiplication map $\mathbf{m}: K \times A \times N \rightarrow G$ is smooth as well.

In order to prove the second assertion note that the tangent mapping

$$
T_{(\mathbf{1}, \mathbf{1}, \mathbf{1})} \mathbf{m}: \mathfrak{k} \times \mathfrak{a} \times \mathfrak{n} \rightarrow \mathfrak{g}
$$


is given by $(X, Y, Z) \mapsto X+Y+Z$; hence $\mathbf{m}$ is a local diffeomorphism if and only if $\mathfrak{k} \dot{+} \mathfrak{a} \dot{+} \mathfrak{n}=\mathfrak{g}$. Since we have already seen that $\mathbf{m}$ is a bijective map, it follows that the latter direct sum decomposition actually holds if and only if $\mathbf{m}$ is a diffeomorphism. Next note that if $\operatorname{dim} \mathcal{H}<\infty$, then we get $\mathfrak{k} \dot{+} \mathfrak{a} \dot{+} \mathfrak{n}=\mathfrak{g}$ by an elementary reasoning (or by the local Iwasawa decomposition for the complex finite-dimensional reductive Lie algebra $\mathfrak{g}$; see, e.g., [Kn96]).

Thus, to complete the proof, it will be enough to show that if the Hilbert space $\mathcal{H}$ is infinite-dimensional, then $\mathfrak{g} \backslash(\mathfrak{k}+\mathfrak{a}+\mathfrak{n}) \neq \emptyset$. In fact, for $j, l \in \mathbb{N}$ denote $h_{j l}=0$ if $j=l$ and $h_{j l}=1 /(j-l)$ if $j \neq l$. Then there exists $W \in \mathcal{B}(\mathcal{H})$ whose matrix with respect to the orthonormal basis $\left\{\xi_{j}\right\}_{j \in \mathbb{N}}$ is $\left(h_{j l}\right)_{j, l \in \mathbb{N}}$, and in addition $W^{*}=-W$ and there exist no operators $Z_{1}, Z_{2} \in \mathfrak{n}$ with $W=Z_{1}-Z_{2}^{*}$ (see Example 4.1 in [Da88]). Now it is easy to see that $\mathrm{i} W \notin \mathfrak{k}+\mathfrak{a}+\mathfrak{n}$. In fact, if $\mathrm{i} W=X+Y+Z$ with $X \in \mathfrak{k}, Y \in \mathfrak{a}$, and $Z \in \mathfrak{n}$, then $\mathrm{i} W=(\mathrm{i} W)^{*}=X^{*}+Y^{*}+Z^{*}=-X+Y+Z^{*}$. Thence $2 \mathrm{i} W=2 Y+Z+Z^{*}$. Since the matrix of $W$ has only zeros on the diagonal, we get $Y=0$, whence $2 \mathrm{i} W=Z+Z^{*}$. Then $W=(-(\mathrm{i} / 2) Z)-(-(\mathrm{i} / 2) Z)^{*}$, and this contradicts one of the above-mentioned properties of $W$. Thus $\mathrm{i} W \in \mathfrak{g} \backslash(\mathfrak{k}+\mathfrak{a}+\mathfrak{n})$, and this completes the proof.

\section{IWASAWA DECOMPOSITIONS FOR INVOLUTIVE BANACH-LiE GROUPS}

In this section we sketch an abstract framework that allows us to investigate Iwasawa decompositions for involutive infinite-dimensional Lie groups modeled on Banach spaces. We will apply these abstract statements in Sections 4, 5, and 6] in the case of the classical Banach-Lie groups associated with norm ideals. The central idea of this abstract approach is that the local Iwasawa decompositions can be constructed out of certain special elements of Lie algebras, which we call Iwasawa regular elements (Definition 2.6).

Preliminaries on local spectral theory. Throughout the paper we let $\mathbb{R}, \mathbb{C}$, and $\mathbb{H}$ stand for the fields of the real, complex, and quaternionic numbers, respectively.

For a real or complex Banach space $\mathfrak{X}$ we denote either by $\mathrm{id}_{\mathfrak{X}}$ or simply by $\mathbf{1}$ the identity map of $\mathfrak{X}$, by $\mathfrak{X}^{*}$ the topological dual of $\mathfrak{X}$, by $\mathcal{B}(\mathfrak{X})$ the algebra of all bounded linear operators on $\mathfrak{X}$ and, when $\mathfrak{X}$ is a complex Banach space, we denote by $\sigma(D)$ the spectrum of $D$ whenever $D \in \mathcal{B}(\mathfrak{X})$. In this case, for every $x \in \mathfrak{X}$ we denote by $\sigma_{D}(x)$ the local spectrum of $x$ with respect to $D$. We recall that $\sigma_{D}(x)$ is a closed subset of $\sigma(D)$ and $w \in \mathbb{C} \backslash \sigma_{D}(x)$ if and only if there exists an open neighborhood $W$ of $w$ and a holomorphic function $\xi: W \rightarrow \mathfrak{X}$ such that $\left(z \operatorname{id}_{\mathfrak{X}}-D\right) \xi(z)=x$ for every $z \in W$. If $F \subseteq \mathbb{C}$ we denote

$$
\mathfrak{X}_{D}(F)=\left\{x \in \mathfrak{X} \mid \sigma_{D}(x) \subseteq F\right\} .
$$

We note that, in the case when $\mathfrak{X}$ has finite dimension $m$, we have

$$
\mathfrak{X}_{D}(F)=\bigoplus_{\lambda \in F \cap \sigma(D)} \operatorname{Ker}\left(\left(D-\lambda \mathrm{id}_{\mathfrak{X}}\right)^{m}\right) \quad \text { for every } F \subseteq \mathbb{C},
$$

while if $\mathfrak{X}$ is a Hilbert space and $D$ is a normal operator with the spectral measure $E_{D}(\cdot)$, then

$$
\mathfrak{X}_{D}(F)=\operatorname{Ran} E_{D}(F) \quad \text { whenever } F \text { is a closed subset of } \mathbb{C} \text {. }
$$

See $\S 12$ in BS01 for a review of the few facts needed from local spectral theory. (More bibliographical details can be found in the Notes to Chapter I in [BS01.) 


\section{Projections on kernels of skew-Hermitian operators.}

Notation 2.1. The following notation will be used throughout the paper:

- For every complex Banach space $\mathfrak{X}$ we denote

$$
\ell_{\mathfrak{X}}^{\infty}(\mathbb{R}):=\left\{f: \mathbb{R} \rightarrow \mathfrak{X} \mid\|f\|_{\infty}:=\sup _{\mathbb{R}}\|f(\cdot)\|<\infty\right\},
$$

which is in turn a complex Banach space.

- We pick a state $\mu: \ell_{\mathbb{C}}^{\infty}(\mathbb{R}) \rightarrow \mathbb{C}$ of the commutative unital $C^{*}$-algebra $\ell_{\mathbb{C}}^{\infty}(\mathbb{R})$ satisfying the translation invariance condition

$$
\left(\forall f \in \ell_{\mathbb{C}}^{\infty}(\mathbb{R})\right)(\forall t \in \mathbb{R}) \quad \mu(f(\cdot))=\mu(f(\cdot+t))
$$

and the symmetry condition

$$
\left(\forall f \in \ell_{\mathbb{C}}^{\infty}(\mathbb{R})\right) \quad \mu(f(\cdot))=\mu(f(-\cdot)) .
$$

(See Problem 7 in Chapter 2 of $\left[\right.$ Pa88].) For every $f \in \ell_{\mathbb{C}}^{\infty}(\mathbb{R})$ we denote $\mu(f(\cdot))=\int_{\mathbb{R}} f(t) \mathrm{d} \mu(t)$.

- For every complex Banach space $\mathfrak{X}$ and every $f \in \ell_{\mathfrak{X}}^{\infty}(\mathbb{R})$ we define $\mu(f)=$ $\int_{\mathbb{R}} f(t) \mathrm{d} \mu(t) \in \mathfrak{X}^{* *}$ by the formula

$$
(\mu(f))(\varphi)=\int_{\mathbb{R}} \varphi(f(t)) \mathrm{d} \mu(t)
$$

whenever $\varphi \in \mathfrak{X}^{*}$ (see [BP07]).

Definition 2.2. Let $\mathfrak{X}_{0}$ be a real Banach space with the complexification

$$
\mathfrak{X}=\left(\mathfrak{X}_{0}\right)^{\mathbb{C}}=\mathfrak{X}_{0}+\mathrm{i} \mathfrak{X}_{0},
$$

which is a complex Banach space with the norm given by

$$
\left\|y_{1}+\mathrm{i} y_{2}\right\|:=\sup _{t \in[0,2 \pi]}\left\|(\cos t) y_{1}+(\sin t) y_{2}\right\| \text { for all } y_{1}, y_{2} \in \mathfrak{X}_{0} .
$$

Let $A: \mathfrak{X}_{0} \rightarrow \mathfrak{X}_{0}$ be a bounded linear operator such that $\sup \|\exp (t A)\|<\infty$, and denote also by $A: \mathfrak{X} \rightarrow \mathfrak{X}$ the $\mathbb{C}$-linear extension of $A: \mathfrak{X}_{0} \rightarrow \mathfrak{X}_{0}$. In this case we define

$$
\mathfrak{X}_{A}^{+}:=\mathfrak{X}_{A}(\mathrm{i}[0, \infty))=\left\{y \in \mathfrak{X} \mid \limsup _{t \rightarrow \infty} \frac{1}{t} \log \|(\exp (\mathrm{i} t A)) y\| \leq 0\right\}
$$

(see also Remark 1.3 in [Be05]). Now assume that $\mathfrak{X}_{0}$ is a reflexive Banach space. Then $\mathfrak{X}$ will be a reflexive complex Banach space, and there exists a bounded linear operator $\mathcal{D}_{\mathfrak{X}, A}: \mathfrak{X} \rightarrow \mathfrak{X}$ defined by

$$
(\forall y \in \mathfrak{X}) \quad \mathcal{D}_{\mathfrak{X}, A} y=\int_{\mathbb{R}}(\exp (t A)) y \mathrm{~d} \mu(t) .
$$

It is easy to see that $\mathcal{D}_{\mathfrak{X}, A} \mathfrak{X}_{0} \subseteq \mathfrak{X}_{0}$, and we shall define $\mathcal{D}_{\mathfrak{X}_{0}, A}:=\left.\mathcal{D}_{\mathfrak{X}, A}\right|_{\mathfrak{X}_{0}}: \mathfrak{X}_{0} \rightarrow \mathfrak{X}_{0}$.

In addition, we define $\mathfrak{X}_{A}^{0,+}:=\mathfrak{X}_{A}^{+} \cap \operatorname{Ker} \mathcal{D}_{\mathfrak{X}, A}$.

Remark 2.3. In the setting of Definition 2.2 we have

$$
\left(\mathcal{D}_{\mathfrak{X}, A}\right)^{2}=\mathcal{D}_{\mathfrak{X}, A}, \quad \operatorname{Ran} \mathcal{D}_{\mathfrak{X}, A}=\operatorname{Ker} A \subseteq \mathfrak{X}, \quad \text { and } \quad \operatorname{Ran} \mathcal{D}_{\mathfrak{X}_{0}, A}=(\operatorname{Ker} A) \cap \mathfrak{X}_{0}
$$
(see [BP07]). 


\section{Elliptic involutive Banach-Lie algebras and abstract Iwasawa decompo- sitions.}

Definition 2.4. Let $\mathfrak{g}_{0}$ be an involutive real or complex Banach-Lie algebra, that is, $\mathfrak{g}_{0}$ is equipped with a continuous linear mapping $X \mapsto X^{*}$ such that $\left(X^{*}\right)^{*}=X$ and $[X, Y]^{*}=-\left[X^{*}, Y^{*}\right]$ whenever $X, Y \in \mathfrak{g}_{0}$. If $\mathfrak{g}_{0}$ is a complex Banach-Lie algebra, then we assume in addition that $(\mathrm{i} X)^{*}=-\mathrm{i} X^{*}$ for all $X \in \mathfrak{g}_{0}$.

We say that $\mathfrak{g}_{0}$ is an elliptic involutive Banach-Lie algebra if $\left\|\exp \left(t \cdot \operatorname{ad}_{\mathfrak{g}_{0}} X\right)\right\| \leq 1$ whenever $t \in \mathbb{R}$ and $X=-X^{*} \in \mathfrak{g}_{0}$.

Remark 2.5. In the special case of the canonically involutive real Banach-Lie algebras (that is, $X^{*}=-X$ for all $X \in \mathfrak{g}_{0}$ ) the above Definition 2.4 coincides with Definition IV.3 in [Nee02b] (or Definition 8.24 in [Be06]).

Definition 2.6. Let $\mathfrak{g}_{0}$ be an elliptic real Banach-Lie algebra with the complexification $\mathfrak{g}$, and denote $\mathfrak{p}_{0}:=\left\{X \in \mathfrak{g}_{0} \mid X^{*}=X\right\}$. An Iwasawa decomposition of $\mathfrak{g}_{0}$ is a direct sum decomposition

$$
\mathfrak{g}_{0}=\mathfrak{k}_{0} \dot{+} \mathfrak{a}_{0} \dot{+} \mathfrak{n}_{0}
$$

satisfying the following conditions:

(j) We have $\mathfrak{k}_{0}=\left\{X \in \mathfrak{g}_{0} \mid X^{*}=-X\right\}$.

(jj) The term $\mathfrak{a}_{0}$ is a linear subspace of $\mathfrak{p}_{0}$ such that $\left[\mathfrak{a}_{0}, \mathfrak{a}_{0}\right]=\{0\}$.

(jjj) There exists $X_{0} \in \mathfrak{a}_{0}$ such that $\mathfrak{a}_{0}=\mathfrak{a}_{X_{0}}$ and $\mathfrak{n}_{0}=\mathfrak{n}_{X_{0}}$, where

$$
\mathfrak{a}_{X_{0}}=\mathfrak{p}_{0} \cap \operatorname{Ker}\left(\operatorname{ad} X_{0}\right) \quad \text { and } \quad \mathfrak{n}_{X_{0}}=\mathfrak{g}_{0} \cap \mathfrak{g}_{\mathrm{ad}\left(-\mathrm{i} X_{0}\right)}^{0,+}
$$

In this case we say that $X_{0}$ is an Iwasawa regular element of $\mathfrak{g}_{0}$ and (2.1) is the Iwasawa decomposition of $\mathfrak{g}_{0}$ associated with $X_{0}$. In the case when the conditions (2.1), (j), and (jjj) are satisfied, we say that $X_{0}$ is an Iwasawa quasi-regular element (and (2.1) is still called the Iwasawa decomposition of $\mathfrak{g}_{0}$ associated with $X_{0}$ ).

Now let us assume that $G$ is a connected Banach-Lie group with $\mathbf{L}(G)=\mathfrak{g}_{0}$ and $K, A$, and $N$ are the connected Banach-Lie groups which are subgroups of $G$ and correspond to the Lie algebras $\mathfrak{k}_{0}, \mathfrak{a}_{0}$, and $\mathfrak{n}_{0}$, respectively. If the mapping

$$
\mathbf{m}: K \times A \times N \rightarrow G, \quad(k, a, n) \mapsto k a n
$$

is a diffeomorphism, then we say that this mapping is the global Iwasawa decomposition of $G$ corresponding to (2.1).

Remark 2.7. In the setting of Definition 2.6, if $X_{0}$ is an Iwasawa regular element, then it is easy to see that $\mathfrak{a}_{X_{0}}$ is a maximal linear subspace of $\mathfrak{p}_{0}$ such that $\left[\mathfrak{a}_{X_{0}}, \mathfrak{a}_{X_{0}}\right]=\{0\}$, while $\mathfrak{n}_{X_{0}}$ is a closed subalgebra of $\mathfrak{g}_{0}$ (see also Be05]).

Remark 2.8. In the setting of Definition 2.6, it is easy to see that all of the groups $K, A$, and $N$ are Banach-Lie subgroups of $G$. (See Up85] or [Be06] for details on the latter notion.)

Proposition 2.9. In the setting of Definition 2.6, let us assume that the BanachLie algebra $\mathfrak{g}_{0}$ is actually an elliptic involutive complex Banach-Lie algebra. Then 
for every $X \in \mathfrak{p}_{0}$ and every closed subset $F$ of $\mathbb{R}$ we have

$$
\mathfrak{g}_{0} \cap \mathfrak{g}_{\operatorname{ad}_{\mathfrak{g}} X}(F)=\left(\mathfrak{g}_{0}\right)_{\operatorname{ad}_{\mathfrak{g}_{0}} X}(F) .
$$

Proof. By the hypothesis that $\mathfrak{g}_{0}$ is an elliptic Banach-Lie algebra, it follows that $\operatorname{ad}_{\mathfrak{g}_{0}} X: \mathfrak{g}_{0} \rightarrow \mathfrak{g}_{0}$ is a Hermitian operator (see Definition 5.23 in [Be06]). If $\mathfrak{g}$ stands for the complexification of $\mathfrak{g}_{0}$, then $\operatorname{ad}_{\mathfrak{g}} X: \mathfrak{g} \rightarrow \mathfrak{g}$ is Hermitian as well.

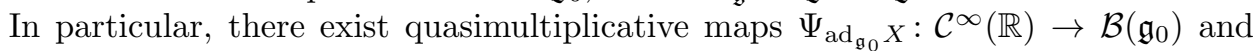
$\Psi_{\mathrm{ad}_{\mathfrak{g}} X}: \mathcal{C}^{\infty}(\mathbb{R}) \rightarrow \mathcal{B}(\mathfrak{g})$ such that $\Psi_{\operatorname{ad}_{\mathfrak{g}_{0}} X}\left(\operatorname{id}_{\mathbb{R}}\right)=\operatorname{ad}_{\mathfrak{g}_{0}} X$ and $\Psi_{\operatorname{ad}_{\mathfrak{g}} X}\left(\operatorname{id}_{\mathbb{R}}\right)=\operatorname{ad}_{\mathfrak{g}} X$, respectively. The maps $\Psi_{\mathrm{ad}_{\mathfrak{g}} X}$ and $\Psi_{\mathrm{ad}_{\mathfrak{g}} X}$ can be constructed by the Weyl functional calculus as in Example 5.25 in [Be06].

Now let $\iota: \mathfrak{g}_{0} \hookrightarrow \mathfrak{g}$ be the inclusion map. Then $\iota(X)=X$, so Remark 5.19 in [Be06] shows that for every closed set $F \subseteq \mathbb{R}$ we have $\iota\left(\left(\mathfrak{g}_{0}\right)_{\operatorname{ad}_{\mathfrak{g}_{0}} X}(F)\right) \subseteq \mathfrak{g}_{\mathrm{ad}_{\mathfrak{g}} X}(F)$, whence $\left(\mathfrak{g}_{0}\right)_{\operatorname{ad}_{\mathfrak{g}_{0}} X}(F) \subseteq \mathfrak{g}_{0} \cap \mathfrak{g}_{\mathrm{ad}_{\mathfrak{g}} X}(F)$.

To prove the converse inclusion, denote by $\kappa: \mathfrak{g} \rightarrow \mathfrak{g}$ the conjugation on $\mathfrak{g}$ whose fixed point set is $\mathfrak{g}_{0}$, and then define $\pi: \mathfrak{g} \rightarrow \mathfrak{g}_{0}, \pi(Z)=(Z+\kappa(Z)) / 2$. Then $\pi(X)=X$, whence $\pi \circ\left(\operatorname{ad}_{\mathfrak{g}} X\right)=\left(\operatorname{ad}_{\mathfrak{g}_{0}} X\right) \circ \pi$. Now Remark 5.19 in Be06] again shows that for every closed subset $F$ of $\mathbb{R}$ we have $\pi\left(\mathfrak{g}_{\mathrm{ad} X}(F)\right) \subseteq \mathfrak{g}_{\mathrm{ad}_{\mathfrak{g}_{0}} X}(F)$. Thence $\mathfrak{g}_{0} \cap \mathfrak{g}_{\mathrm{ad} X}(F) \subseteq \mathfrak{g}_{\operatorname{ad}_{\mathfrak{g}_{0}} X}(F)$, and we are done.

Remark 2.10. In the special case when $\operatorname{dim} \mathfrak{g}_{0}<\infty$ and $F$ is a certain finite subset of $\mathbb{R}_{+}$, the conclusion of our Proposition 2.9 was obtained in Chapter VI, $\S 6$ of He01 by using the structure theory of finite-dimensional complex semisimple Lie algebras.

Proposition 2.11. Let $\widetilde{\mathfrak{g}}$ be an elliptic complex Banach-Lie algebra whose underlying Banach space is reflexive, and pick $X_{0}=X_{0}^{*} \in \widetilde{\mathfrak{g}}$. Assume that we have a bounded linear operator $\widetilde{\mathcal{T}}: \widetilde{\mathfrak{g}} \rightarrow \widetilde{\mathfrak{g}}$ such that

$$
\begin{aligned}
\widetilde{\mathcal{T}}^{2} & =\widetilde{\mathcal{T}}, \\
\operatorname{Ran} \widetilde{\mathcal{T}} & =\widetilde{\mathfrak{g}}_{\mathrm{ad} X_{0}}\left(\mathbb{R}_{+}\right), \\
\operatorname{Ran}(\mathbf{1}-\widetilde{\mathcal{T}}) & \subseteq \widetilde{\mathfrak{g}}_{\mathrm{ad} X_{0}}\left(-\mathbb{R}_{+}\right) .
\end{aligned}
$$

Then $X_{0}$ is an Iwasawa quasi-regular element of $\widetilde{\mathfrak{g}}$. Now let

$$
\widetilde{\mathfrak{g}}=\widetilde{\mathfrak{k}} \dot{+} \widetilde{\mathfrak{a}} \dot{+} \tilde{\mathfrak{n}}
$$

be the Iwasawa decomposition of $\widetilde{\mathfrak{g}}$ associated with $X_{0}$, and for $\widetilde{\mathfrak{s}} \in\{\widetilde{\mathfrak{k}}, \mathfrak{\mathfrak { a }}, \widetilde{\mathfrak{n}}\}$, denote by $p_{\mathfrak{s}}: \widetilde{\mathfrak{g}} \rightarrow \widetilde{\mathfrak{s}}$ the linear projections corresponding to the direct sum decomposition (2.5). Then for all $X \in \widetilde{\mathfrak{g}}$ we have

$$
\begin{aligned}
& p_{\tilde{\mathfrak{k}}}(X)=(\mathbf{1}-\widetilde{\mathcal{T}}) X-((\mathbf{1}-\widetilde{\mathcal{T}}) X)^{*}+\frac{1}{2}\left(\mathcal{D}_{\tilde{\mathfrak{g}}, \operatorname{ad}\left(-\mathrm{i} X_{0}\right)} X-\left(\mathcal{D}_{\tilde{\mathfrak{g}}, \operatorname{ad}\left(-\mathrm{i} X_{0}\right)} X\right)^{*}\right), \\
& p_{\tilde{\mathfrak{a}}}(X)=\frac{1}{2}\left(\mathcal{D}_{\widetilde{\mathfrak{g}}, \operatorname{ad}\left(-\mathrm{i} X_{0}\right)} X+\left(\mathcal{D}_{\tilde{\mathfrak{g}}, \operatorname{ad}\left(-\mathrm{i} X_{0}\right)} X\right)^{*}\right), \text { and } \\
& p_{\tilde{\mathfrak{n}}}(X)=\left(\widetilde{\mathcal{T}}-\mathcal{D}_{\widetilde{\mathfrak{g}}, \operatorname{ad}\left(-\mathrm{i} X_{0}\right)}\right) X+((\mathbf{1}-\widetilde{\mathcal{T}}) X)^{*} .
\end{aligned}
$$

Proof. To begin with, recall that

$$
\begin{aligned}
\widetilde{\mathfrak{k}} & =\left\{X \in \widetilde{\mathfrak{g}} \mid X^{*}=-X\right\}, \\
\widetilde{\mathfrak{a}} & =\left\{X \in \widetilde{\mathfrak{g}} \mid X^{*}=X \text { and }\left[X_{0}, X\right]=0\right\}, \text { and } \\
\tilde{\mathfrak{n}} & =\widetilde{\mathfrak{g}}_{\mathrm{ad}\left(-\mathrm{i} X_{0}\right)}^{0,+}=\widetilde{\mathfrak{g}}_{\mathrm{ad}} X_{0}\left(\mathbb{R}_{+}\right) \cap \operatorname{Ker} \mathcal{D}_{\tilde{\mathfrak{g}}, \operatorname{ad}\left(-\mathrm{i} X_{0}\right)},
\end{aligned}
$$


where the latter two equalities follow by Proposition 2.9. Definition 2.2. and Definition 2.6. It is straightforward to check that $\widetilde{\mathfrak{k}} \cap(\widetilde{\mathfrak{a}}+\widetilde{\mathfrak{n}})=\widetilde{\mathfrak{a}} \cap \widetilde{\mathfrak{n}}=\{0\}$, whence

$$
\widetilde{\mathfrak{k}} \cap(\widetilde{\mathfrak{a}}+\widetilde{\mathfrak{n}})=\widetilde{\mathfrak{a}} \cap(\widetilde{\mathfrak{k}}+\widetilde{\mathfrak{n}})=\widetilde{\mathfrak{n}} \cap(\widetilde{\mathfrak{k}}+\widetilde{\mathfrak{a}})=\{0\},
$$

and it remains to prove that $\widetilde{\mathfrak{k}}+\widetilde{\mathfrak{a}}+\widetilde{\mathfrak{n}}=\widetilde{\mathfrak{g}}$.

For this purpose, first note that $\mathcal{D}_{\tilde{\mathfrak{g}}, \operatorname{ad}\left(-\mathrm{i} X_{0}\right)}$ and $\widetilde{\mathcal{T}}$ are idempotent operators on $\widetilde{\mathfrak{g}}$ satisfying

hence

$$
\operatorname{Ran} \mathcal{D}_{\widetilde{\mathfrak{g}}, \operatorname{ad}\left(-\mathrm{i} X_{0}\right)} \subseteq \operatorname{Ran} \widetilde{\mathcal{T}}
$$

Now let $X \in \tilde{\mathfrak{g}}$ be arbitrary and denote by $X_{\mathfrak{k}}, X_{\mathfrak{a}}$, and $X_{\mathfrak{n}}$ the right-hand sides of the wished-for formulas for $p_{\mathfrak{\mathfrak { k }}}(X), p_{\mathfrak{a}}(X)$, and $p_{\mathfrak{\mathfrak { n }}}(X)$, respectively. Thus we have to prove that $p_{\mathfrak{s}}(X)=X_{\mathfrak{s}}$ for $\mathfrak{s} \in\{\mathfrak{k}, \mathfrak{a}, \mathfrak{n}\}$. Moreover, it is clear that $X=X_{\mathfrak{k}}+X_{\mathfrak{a}}+X_{\mathfrak{n}}$, so it will be enough to check that $X_{\mathfrak{k}} \in \widetilde{\mathfrak{k}}, X_{\mathfrak{a}} \in \widetilde{\mathfrak{a}}$, and $X_{\mathfrak{n}} \in \widetilde{\mathfrak{n}}$.

It follows at once by (2.6) that $X_{\mathfrak{k}} \in \widetilde{\mathfrak{k}}$. To see that $X_{\mathfrak{a}} \in \widetilde{\mathfrak{a}}$, first note that $X_{\mathfrak{a}}^{*}=X_{\mathfrak{a}}$. On the other hand, we have $\left[X_{0}, \mathcal{D}_{\tilde{\mathfrak{g}}, \mathrm{ad}\left(-\mathrm{i} X_{0}\right)} X\right]=0$ (see Remark 2.3). Since $X_{0}=X_{0}^{*}$, it then follows that $\left[X_{0},\left(\mathcal{D}_{\mathfrak{\mathfrak { g }}, \mathrm{ad}\left(-\mathrm{i} X_{0}\right)} X\right)^{*}\right]=0$, whence $\left[X_{0}, X_{\mathfrak{a}}\right]=0$. Thus $X_{\mathfrak{a}} \in \tilde{\mathfrak{a}}$.

It remains to show that $X_{\mathfrak{n}} \in \tilde{\mathfrak{n}}$. To this end, first note that

$$
\mathcal{D}_{\widetilde{\mathfrak{g}}, \operatorname{ad}\left(-\mathrm{i} X_{0}\right)}\left(\widetilde{\mathcal{T}}-\mathcal{D}_{\widetilde{\mathfrak{g}}, \operatorname{ad}\left(-\mathrm{i} X_{0}\right)}\right) X=0
$$

and

$$
\mathcal{D}_{\widetilde{\mathfrak{g}}, \operatorname{ad}\left(-\mathrm{i} X_{0}\right)}\left(((\mathbf{1}-\widetilde{\mathcal{T}}) X)^{*}\right)=\left(\mathcal{D}_{\widetilde{\mathfrak{g}}, \operatorname{ad}\left(-\mathrm{i} X_{0}\right)}(\mathbf{1}-\widetilde{\mathcal{T}}) X\right)^{*}=0
$$

by (2.9) and the fact that $\left(\mathcal{D}_{\mathfrak{\mathfrak { g }}, \mathrm{ad}\left(-\mathrm{i} X_{0}\right)} Y\right)^{2}=\mathcal{D}_{\mathfrak{\mathfrak { g }}, \mathrm{ad}\left(-\mathrm{i} X_{0}\right)} Y$. (We also used the fact that $\mathcal{D}_{\tilde{\mathfrak{g}}, \text { ad }\left(-\mathrm{i} X_{0}\right)}\left(Y^{*}\right)=\left(\mathcal{D}_{\tilde{\mathfrak{g}}, \operatorname{ad}\left(-\mathrm{i} X_{0}\right)} Y\right)^{*}$ whenever $Y \in \widetilde{\mathfrak{g}}$, which is a consequence of Definition 2.2 since $X_{0}^{*}=X_{0}$.) It then follows that $\mathcal{D}_{\widetilde{\mathfrak{g}}, \text { ad }\left(-\mathrm{i} X_{0}\right)}\left(X_{\mathfrak{n}}\right)=0$. Thus, according to (2.8), we still have to prove that $X_{\mathfrak{n}} \in \widetilde{\mathfrak{g}}_{\mathrm{ad}\left(-\mathrm{i} X_{0}\right)}\left(\mathbb{R}_{+}\right)$. For this purpose we are going to show that both terms in the expression of $X_{\mathfrak{n}}$ belong to $\widetilde{\mathfrak{g}}_{\mathrm{ad}} X_{0}\left(\mathbb{R}_{+}\right)$. In fact, by (2.9) and (2.3) we get

$$
\left(\widetilde{\mathcal{T}}-\mathcal{D}_{\tilde{\mathfrak{g}}, \operatorname{ad}\left(-\mathrm{i} X_{0}\right)}\right) X=\widetilde{\mathcal{T}}\left(\mathbf{1}-\mathcal{D}_{\tilde{\mathfrak{g}}, \operatorname{ad}\left(-\mathrm{i} X_{0}\right)}\right) X \in \operatorname{Ran} \widetilde{\mathcal{T}} \subseteq \widetilde{\mathfrak{g}}_{\mathrm{ad} X}\left(\mathbb{R}_{+}\right) .
$$

On the other hand, the mapping $\kappa: \widetilde{\mathfrak{g}} \rightarrow \widetilde{\mathfrak{g}}, Y \mapsto Y^{*}$ has the property

$$
\theta \circ \operatorname{ad} X_{0}=-\operatorname{ad} X_{0} \circ \theta
$$

since $X_{0}^{*}=X_{0}$. Then Proposition 5.22 in $[\mathrm{Be} 06]$ shows that

$$
\kappa\left(\widetilde{\mathfrak{g}}_{\mathrm{ad} X_{0}}\left(-\mathbb{R}_{+}\right)\right) \subseteq \widetilde{\mathfrak{g}}_{\mathrm{ad} X_{0}}\left(\mathbb{R}_{+}\right),
$$

whence by (2.4) we get $((\mathbf{1}-\widetilde{\mathcal{T}}) X)^{*}=\kappa((\mathbf{1}-\widetilde{\mathcal{T}}) X) \in \widetilde{\mathfrak{g}}_{\mathrm{ad} X_{0}}\left(\mathbb{R}_{+}\right)$. Consequently

$$
X_{\mathfrak{n}}=\left(\widetilde{\mathcal{T}}-\mathcal{D}_{\tilde{\mathfrak{g}}, \operatorname{ad}\left(-\mathrm{i} X_{0}\right)}\right) X+((\mathbf{1}-\widetilde{\mathcal{T}}) X)^{*} \in \tilde{\mathfrak{g}}_{\mathrm{ad}} X_{0}\left(\mathbb{R}_{+}\right),
$$

and the proof is complete.

Corollary 2.12. Assume the setting of Proposition 2.11 and let $\mathfrak{g}$ be a closed involutive complex subalgebra of $\widetilde{\mathfrak{g}}$ such that

$$
X_{0} \in \mathfrak{g} \quad \text { and } \quad \tilde{\mathcal{T}}(\mathfrak{g}) \subseteq \mathfrak{g} .
$$

Then $X_{0}$ is an Iwasawa quasi-regular element of $\mathfrak{g}$ and the Iwasawa decomposition of $\mathfrak{g}$ associated with $X_{0}$ is $\mathfrak{g}=(\widetilde{\mathfrak{k}} \cap \mathfrak{g}) \dot{+}(\widetilde{\mathfrak{a}} \cap \mathfrak{g}) \dot{+}(\widetilde{\mathfrak{n}} \cap \mathfrak{g})$. 
Proof. Since $\tilde{\mathcal{T}}(\mathfrak{g}) \subseteq \mathfrak{g}$ and $\mathcal{D}_{\tilde{\mathfrak{g}}, \text { ad }\left(-\mathrm{i} X_{0}\right)}(\mathfrak{g}) \subseteq \mathfrak{g}$, it follows by Proposition 2.11 that $p_{\mathfrak{\mathfrak { k }}}(\mathfrak{g}) \subseteq \mathfrak{g}, p_{\mathfrak{\mathfrak { a }}}(\mathfrak{g}) \subseteq \mathfrak{g}$, and $p_{\mathfrak{\mathfrak { n }}}(\mathfrak{g}) \subseteq \mathfrak{g}$. It then follows by the direct sum decomposition (2.5) that $\mathfrak{g}=(\widetilde{\mathfrak{k}} \cap \mathfrak{g}) \dot{+}(\widetilde{\mathfrak{a}} \cap \mathfrak{g}) \dot{+}(\widetilde{\mathfrak{n}} \cap \mathfrak{g})$. Thus, to conclude the proof, it remains to prove that

$$
\widetilde{\mathfrak{a}} \cap \mathfrak{g}=\left\{X \in \mathfrak{g} \mid X^{*}=X \text { and }\left[X, X_{0}\right]=0\right\} \quad \text { and } \quad \widetilde{\mathfrak{n}} \cap \mathfrak{g}=\mathfrak{g}_{\mathrm{ad}\left(-\mathrm{i} X_{0}\right)}^{0,+}
$$

(see also Proposition 2.9). The equality involving $\widetilde{\mathfrak{a}} \cap \mathfrak{g}$ is obvious. To prove the equality involving $\tilde{\mathfrak{n}} \cap \mathfrak{g}$, just note that by Proposition 2.9 we have

$$
\begin{aligned}
\tilde{\mathfrak{n}} \cap \mathfrak{g} & =\widetilde{\mathfrak{g}}_{\mathrm{ad}\left(-\mathrm{i} X_{0}\right)}^{0,+} \cap \mathfrak{g}=\widetilde{\mathfrak{g}}_{\mathrm{ad}\left(-\mathrm{i} X_{0}\right)}\left(\mathbb{R}_{+}\right) \cap\left(\operatorname{Ker} \mathcal{D}_{\widetilde{\mathfrak{g}}, \operatorname{ad}\left(-\mathrm{i} X_{0}\right)}\right) \cap \mathfrak{g} \\
& =\mathfrak{g}_{\mathrm{ad}\left(-\mathrm{i} X_{0}\right)}\left(\mathbb{R}_{+}\right) \cap\left(\operatorname{Ker} \mathcal{D}_{\mathfrak{g}, \operatorname{ad}\left(-\mathrm{i} X_{0}\right)}\right)=\mathfrak{g}_{\mathrm{ad}\left(-\mathrm{i} X_{0}\right)}^{0,+}
\end{aligned}
$$

and this completes the proof.

Corollary 2.13. Assume the setting of Proposition 2.11 and let $\mathfrak{g}_{0}$ be a closed involutive real subalgebra of $\widetilde{\mathfrak{g}}$ such that

$$
X_{0} \in \mathfrak{g}_{0}, \quad \tilde{\mathcal{T}}\left(\mathfrak{g}_{0}\right) \subseteq \mathfrak{g}_{0}, \quad \text { and } \quad \mathfrak{g}_{0} \cap \mathfrak{i g}_{0}=\{0\} .
$$

Then $X_{0}$ is an Iwasawa quasi-regular element of $\mathfrak{g}$ and the Iwasawa decomposition of $\mathfrak{g}$ associated with $X_{0}$ is $\mathfrak{g}_{0}=\left(\widetilde{\mathfrak{k}} \cap \mathfrak{g}_{0}\right) \dot{+}\left(\widetilde{\mathfrak{a}} \cap \mathfrak{g}_{0}\right) \dot{+}\left(\widetilde{\mathfrak{n}} \cap \mathfrak{g}_{0}\right)$.

Proof. Denote $\mathfrak{g}:=\mathfrak{g}_{0}+\mathfrak{i g}_{0}(\subseteq \widetilde{\mathfrak{g}})$. Since $\mathfrak{g}_{0} \cap \mathfrak{i} \mathfrak{g}_{0}=\{0\}$, it follows that $\mathfrak{g}$ is isomorphic to the complexification of $\mathfrak{g}_{0}$ (as a complex involutive Banach-Lie algebra).

We have $X_{0} \in \mathfrak{g}_{0} \subseteq \mathfrak{g}$ and $\widetilde{\mathcal{T}}(\mathfrak{g})=\widetilde{\mathcal{T}}\left(\mathfrak{g}_{0}+\mathrm{i} \mathfrak{g}_{0}\right) \subseteq \mathfrak{g}_{0}+\mathrm{i} \mathfrak{g}_{0}=\mathfrak{g}$; hence Corollary 2.12 shows that $X_{0}$ is Iwasawa regular in $\mathfrak{g}$ and the corresponding Iwasawa decomposition of $\mathfrak{g}$ is $\mathfrak{g}=(\widetilde{\mathfrak{k}} \cap \mathfrak{g}) \dot{+}(\widetilde{\mathfrak{a}} \cap \mathfrak{g}) \dot{+}(\widetilde{\mathfrak{n}} \cap \mathfrak{g})$.

In particular we get $\mathfrak{g}_{\mathrm{ad}\left(-\mathrm{i} X_{0}\right)}^{0,+}=\widetilde{\mathfrak{n}} \cap \mathfrak{g}$, whence

$$
\mathfrak{g}_{0} \cap \mathfrak{g}_{\mathrm{ad}\left(-\mathrm{i} X_{0}\right)}^{0,+}=\widetilde{\mathfrak{n}} \cap \mathfrak{g}_{0} .
$$

On the other hand, it is obvious that

$\left\{X \in \mathfrak{g}_{0} \mid X^{*}=-X\right\}=\tilde{\mathfrak{k}} \cap \mathfrak{g}_{0} \quad$ and $\quad\left\{X \in \mathfrak{g}_{0} \mid X^{*}=X\right.$ and $\left.\left[X_{0}, X\right]=0\right\}=\widetilde{\mathfrak{a}} \cap \mathfrak{g}_{0} ;$ hence the conclusion will follow by $\mathfrak{g}_{0}=\left(\widetilde{\mathfrak{k}} \cap \mathfrak{g}_{0}\right) \dot{+}\left(\widetilde{\mathfrak{a}} \cap \mathfrak{g}_{0}\right) \dot{+}\left(\widetilde{\mathfrak{n}} \cap \mathfrak{g}_{0}\right)$, and this direct sum decomposition can be obtained just as in the proof of Corollary 2.12. Indeed, we have $\widetilde{\mathcal{T}}\left(\mathfrak{g}_{0}\right) \subseteq \mathfrak{g}_{0}$ and $\mathcal{D}_{\tilde{\mathfrak{g}}, \text { ad }\left(-\mathrm{i} X_{0}\right)}\left(\mathfrak{g}_{0}\right) \subseteq \mathfrak{g}_{0}$. Then we can use Proposition 2.11 to show that $p_{\mathfrak{\mathfrak { k }}}\left(\mathfrak{g}_{0}\right) \subseteq \mathfrak{g}_{0}, p_{\widetilde{\mathfrak{a}}}\left(\mathfrak{g}_{0}\right) \subseteq \mathfrak{g}_{0}$, and $p_{\widetilde{\mathfrak{n}}}\left(\mathfrak{g}_{0}\right) \subseteq \mathfrak{g}_{0}$. Since $\widetilde{\mathfrak{g}}=\widetilde{\mathfrak{k}}+\widetilde{\mathfrak{a}}+\widetilde{\mathfrak{n}}$ by the hypothesis, it then follows that $\mathfrak{g}_{0}=\left(\widetilde{\mathfrak{k}} \cap \mathfrak{g}_{0}\right) \dot{+}\left(\widetilde{\mathfrak{a}} \cap \mathfrak{g}_{0}\right) \dot{+}\left(\widetilde{\mathfrak{n}} \cap \mathfrak{g}_{0}\right)$.

\section{Inductive limits of Iwasawa decompositions.}

Lemma 2.14. Let $\widetilde{\Psi}: \widetilde{S}_{1} \rightarrow \widetilde{S}_{2}$ be an open bijective mapping between two topological spaces. Assume that $S_{j}$ is a closed subset of $\widetilde{S}_{j}$ for $j=1,2$ such that $\widetilde{\Psi}\left(S_{1}\right)$ is a dense subset of $S_{2}$. Then $\widetilde{\Psi}\left(S_{1}\right)=S_{2}$.

Proof. We have to prove that $S_{2} \subseteq \widetilde{\Psi}\left(S_{1}\right)$. The hypothesis that $\widetilde{\Psi}: \widetilde{S}_{1} \rightarrow \widetilde{S}_{2}$ is an open bijection implies that its inverse $\widetilde{\Psi}^{-1}: \widetilde{S}_{2} \rightarrow \widetilde{S}_{1}$ is continuous. Then by using the other hypothesis, namely $\overline{\widetilde{\Psi}\left(S_{1}\right)}=S_{2}$, we get

$$
\widetilde{\Psi}^{-1}\left(S_{2}\right)=\widetilde{\Psi}^{-1}\left(\overline{\widetilde{\Psi}\left(S_{1}\right)}\right) \subseteq \overline{\widetilde{\Psi}^{-1}\left(\widetilde{\Psi}\left(S_{1}\right)\right)}=\overline{S_{1}}=S_{1},
$$

which concludes the proof since $\widetilde{\Psi}$ is a bijection. 
Proposition 2.15. Let $\widetilde{G}$ be a Banach-Lie group and assume that $\widetilde{K}, \widetilde{A}$, and $\widetilde{N}$ are Banach-Lie subgroups of $\widetilde{G}$ such that the multiplication map $\widetilde{\boldsymbol{m}}: \widetilde{K} \times \widetilde{A} \times \widetilde{N} \rightarrow \widetilde{G}$, $(\widetilde{k}, \widetilde{a}, \widetilde{n}) \mapsto \widetilde{k} \widetilde{a} \widetilde{n}$ is a diffeomorphism.

Then let $G, K, A$, and $N$ be four connected Lie subgroups of $\widetilde{G}$ with $\mathbf{L}(G)=\mathfrak{g}$, $\mathbf{L}(K)=\mathfrak{k}, \mathbf{L}(A)=\mathfrak{a}$, and $\mathbf{L}(N)=\mathfrak{n}$, and assume that $\mathfrak{g}$ is an elliptic real BanachLie algebra and $X_{0} \in \mathfrak{g}$ is an Iwasawa regular element such that the following conditions are satisfied:

(j) We have $K \subseteq \widetilde{K} \cap G, A \subseteq \widetilde{A} \cap G, N \subseteq \widetilde{N} \cap G$, and $G$ is a Banach-Lie subgroup of $\widetilde{G}$.

(jj) The Iwasawa decomposition of $\mathfrak{g}$ with respect to $X_{0}$ is $\mathfrak{g}=\mathfrak{k} \dot{+} \mathfrak{a} \dot{+} \mathfrak{n}$.

(jjj) There exists a family $\left\{\mathfrak{g}_{i}\right\}_{i \in I}$ consisting of finite-dimensional reductive subalgebras of $\mathfrak{g}$ such that

- there exists a bounded linear map $\mathcal{E}_{i}: \mathfrak{g} \rightarrow \mathfrak{g}$ such that $\mathcal{E}_{i}\left(X^{*}\right)=\mathcal{E}_{i}(X)^{*}$ for all $X \in \mathfrak{g}$,

- $\left(\mathcal{E}_{i}\right)^{2}=\mathcal{E}_{i}, \operatorname{Ran} \mathcal{E}_{i}=\mathfrak{g}_{i}$, and $\left[\operatorname{Ran}\left(\mathbf{1}-\mathcal{E}_{i}\right), \mathfrak{g}_{i}\right]=\{0\}$,

- $\mathcal{E}_{i}\left(X_{0}\right)$ is an Iwasawa regular element of $\mathfrak{g}_{i}$, and

- the connected subgroup $G_{i}$ of $G$ with $\mathbf{L}\left(G_{i}\right)=\mathfrak{g}_{i}$ is a closed subgroup and a finite-dimensional reductive Lie group

$$
\text { for every } i \in I \text {, and } \overline{\bigcup_{i \in I} \mathfrak{g}_{i}}=\mathfrak{g} \text {. }
$$

Then the mapping $\boldsymbol{m}:=\left.\widetilde{\boldsymbol{m}}\right|_{K \times A \times N}: K \times A \times N \rightarrow G$ is a diffeomorphism. Moreover, $A N=N A$ and both groups $A$ and $N$ are simply connected.

Proof. Since $\widetilde{\mathbf{m}}$ is smooth and $G$ is a Banach-Lie subgroup of $\widetilde{G}$ by hypothesis (j), it follows that $\mathbf{m}$ is a smooth mapping. Then condition (jj) shows that the tangent map of $\mathbf{m}$ at any point of $K \times A \times N$ is an invertible continuous linear operator; hence $\mathbf{m}$ is a local diffeomorphism. On the other hand, $\mathbf{m}$ is injective since $\widetilde{\mathbf{m}}$ is so.

It remains to prove that $\mathbf{m}$ is surjective. To this end let $i \in I$ and denote by $\mathfrak{g}_{i}=\mathfrak{k}_{i} \dot{+} \mathfrak{a}_{i} \dot{+} \mathfrak{n}_{i}$ the Iwasawa decomposition of $\mathfrak{g}_{i}$ with respect to $\mathcal{E}_{i}\left(X_{0}\right)$. Also let $G_{i}=K_{i} A_{i} N_{i}$ be the corresponding global Iwasawa decomposition of the finitedimensional reductive Lie group $G_{i}$ (see [Kn96]). We have

$$
\mathfrak{k}_{i}=\left\{X \in \mathfrak{g}_{i} \mid X^{*}=-X\right\}=\mathfrak{k} \cap \mathfrak{g}_{i} .
$$

Also

$$
\mathfrak{a}_{i}=\left\{X=X^{*} \in \mathfrak{g}_{i} \mid\left[X, \mathcal{E}_{i}\left(X_{0}\right)\right]=0\right\} \subseteq\left\{X=X^{*} \in \mathfrak{g}_{i} \mid\left[X, X_{0}\right]=0\right\}=\mathfrak{a} \cap \mathfrak{g}_{i},
$$

since $\left[\mathfrak{g}_{i}, \operatorname{Ran}\left(\mathbf{1}-\mathcal{E}_{i}\right)\right]=\{0\}$. Finally, by Definitions 2.2 and 2.6 we get

$$
\mathfrak{n}_{i}=\mathfrak{g}_{i} \cap\left(\left(\mathfrak{g}_{i}\right)_{\mathbb{C}}\right)_{\operatorname{ad}\left(-\mathrm{i} P\left(X_{0}\right)\right)}^{0,+} \subseteq \mathfrak{g} \cap\left(\mathfrak{g}_{\mathbb{C}}\right)_{\operatorname{ad}\left(-\mathrm{i} X_{0}\right)}^{0,+} \cap \mathfrak{g}_{i}=\mathfrak{n} \cap \mathfrak{g}_{i},
$$

where $(\bullet)_{\mathbb{C}}$ stands for the complexification of a Lie algebra. Consequently we have $K_{i} \subseteq K \cap G_{i}, A_{i} \subseteq A \cap G_{i}$, and $N_{i} \subseteq N \cap G_{i}$. (Here we use the fact that each of the groups $K_{i}, A_{i}$, and $N_{i}$ is connected since $G_{i}$ is connected and there exists a diffeomorphism $G_{i} \simeq K_{i} \times A_{i} \times N_{i}$.)

Now we are going to apply Lemma 2.14 for the spaces $\widetilde{S}_{1}=\widetilde{K} \times \widetilde{A} \times \widetilde{N}, \widetilde{S} \widetilde{S}_{2}=\widetilde{G}$, $S_{1}=K \times A \times N$, and $S_{2}=G$. The mapping $\widetilde{\mathbf{m}}$ is an open bijection since it is a diffeomorphism. On the other hand,

$$
\mathbf{m}(K \times A \times N) \supseteq \bigcup_{i \in I} \mathbf{m}\left(K_{i} \times A_{i} \times N_{i}\right)=\bigcup_{i \in I} G_{i} ;
$$


hence $\mathbf{m}(K \times A \times N)$ is a dense subset of $G$. (Note that $\bigcup_{i \in I} G_{i}$ is dense in $G$ since $\bigcup_{i \in I} \mathfrak{g}_{i}$ is dense in $\mathfrak{g}$ and $G$ is connected.) Thus Lemma 2.14 applies and shows that $\mathbf{m}(K \times A \times N)=G$, hence $\mathbf{m}: K \times A \times N \rightarrow G$ is a diffeomorphism.

To complete the proof, use the inverse diffeomorphism $\mathbf{m}^{-1}: G \rightarrow K \times A \times N$. Since $\bigcup_{i \in I} G_{i}$ is dense in $G$, it follows that $\bigcup_{i \in I} K_{i}$ is dense in $K, \bigcup_{i \in I} A_{i}$ is dense in $A$, and $\bigcup_{i \in I} N_{i}$ is dense in $N$. Now the conclusion follows since $A_{i} N_{i}=N_{i} A_{i}$ and both groups $A_{i}$ and $N_{i}$ are simply connected for all $i \in I$.

\section{Classical Banach-Lie groups and their Lie algebras}

In this section we introduce the Banach-Lie groups and Lie algebras whose Iwasawa decompositions will be investigated in Sections 4, 5, and 6 and we record a few auxiliary results that will be used in those sections.

Definition 3.1. We denote by $\operatorname{GL}(\mathcal{H})$ the group of all invertible bounded linear operators on the complex Hilbert space $\mathcal{H}$ and by $\mathfrak{I}$ an arbitrary norm ideal of $\mathcal{B}(\mathcal{H})$. We define the following complex Banach-Lie groups and Banach-Lie algebras:

(A) $\operatorname{GL}_{\mathfrak{I}}(\mathcal{H})=\operatorname{GL}(\mathcal{H}) \cap(\mathbf{1}+\mathfrak{I})$ with the Lie algebra

$$
\mathbf{L}\left(\mathrm{GL}_{\mathfrak{I}}(\mathcal{H})\right):=\mathfrak{g l}_{\mathfrak{I}}(\mathcal{H}):=\mathfrak{I}
$$

(B) $\mathrm{O}_{\mathfrak{I}}(\mathcal{H}):=\left\{g \in \mathrm{GL}_{\mathfrak{I}}(\mathcal{H}) \mid g^{-1}=J g^{*} J^{-1}\right\}$ with the Lie algebra

$$
\mathbf{L}\left(\mathrm{O}_{\mathfrak{I}}(\mathcal{H})\right):=\mathfrak{o}_{\mathfrak{I}}(\mathcal{H}):=\left\{x \in \mathfrak{I} \mid x=-J x^{*} J^{-1}\right\},
$$

where $J: \mathcal{H} \rightarrow \mathcal{H}$ is a conjugation (i.e., $J$ is a conjugate-linear isometry satisfying $\left.J^{2}=\mathbf{1}\right)$;

(C) $\operatorname{Sp}_{\mathfrak{I}}(\mathcal{H}):=\left\{g \in \mathrm{GL}_{\mathfrak{I}}(\mathcal{H}) \mid g^{-1}=\widetilde{J} g^{*} \widetilde{J}^{-1}\right\}$ with the Lie algebra

$$
\mathbf{L}\left(\operatorname{Sp}_{\mathfrak{I}}(\mathcal{H})\right):=\mathfrak{s p} \mathfrak{I}_{\mathfrak{I}}(\mathcal{H}):=\left\{x \in \mathfrak{I} \mid x=-\widetilde{J} x^{*} \widetilde{J}^{-1}\right\},
$$

where $\widetilde{J}: \mathcal{H} \rightarrow \mathcal{H}$ is an anti-conjugation (i.e., $\widetilde{J}$ a conjugate-linear isometry satisfying $\left.\widetilde{J}^{2}=-\mathbf{1}\right)$.

We shall say that $\mathrm{GL}_{\mathfrak{I}}(\mathcal{H}), \mathrm{O}_{\mathfrak{I}}(\mathcal{H})$, and $\operatorname{Sp}_{\mathfrak{I}}(\mathcal{H})$ are the classical complex BanachLie groups associated with the operator ideal $\mathfrak{I}$. Similarly, the corresponding Lie algebras are called the classical complex Banach-Lie algebras (associated with I).

When no confusion can occur, we shall denote the groups $\operatorname{GL}_{\mathfrak{I}}(\mathcal{H}), \mathrm{O}_{\mathfrak{I}}(\mathcal{H})$, and $\mathrm{Sp}_{\mathfrak{I}}(\mathcal{H})$ simply by $\mathrm{GL}_{\mathfrak{I}}, \mathrm{O}_{\mathfrak{I}}$, and $\mathrm{Sp}_{\mathfrak{I}}$, respectively, and we shall proceed similarly for the classical complex Lie algebras.

Definition 3.2. We shall use the notation of Definition 3.1 and define the following real Banach-Lie groups and Banach-Lie algebras:

(AI) $\operatorname{GL}_{\mathfrak{I}}(\mathcal{H} ; \mathbb{R})=\left\{g \in \mathrm{GL}_{\mathfrak{I}}(\mathcal{H}) \mid g J=J g\right\}$ with the Lie algebra

$$
\mathbf{L}\left(\operatorname{GL}_{\mathfrak{I}}(\mathcal{H} ; \mathbb{R})\right):=\mathfrak{g l}_{\mathfrak{I}}(\mathcal{H} ; \mathbb{R}):=\{x \in \mathfrak{I} \mid x J=J x\},
$$

where $J: \mathcal{H} \rightarrow \mathcal{H}$ is any conjugation on $\mathcal{H}$;

(AII) $\operatorname{GL}_{\mathfrak{I}}(\mathcal{H} ; \mathbb{H})=\left\{g \in \mathrm{GL}_{\mathfrak{I}}(\mathcal{H}) \mid g \widetilde{J}=\widetilde{J} g\right\}$ with the Lie algebra

$$
\mathbf{L}\left(\operatorname{GL}_{\mathfrak{I}}(\mathcal{H} ; \mathbb{H})\right):=\mathfrak{g l}_{\mathfrak{I}}(\mathcal{H} ; \mathbb{H}):=\{x \in \mathfrak{I} \mid x \widetilde{J}=\widetilde{J} x\},
$$

where $\widetilde{J}: \mathcal{H} \rightarrow \mathcal{H}$ is any anti-conjugation on $\mathcal{H}$; 
(AIII) $\mathrm{U}_{\mathfrak{I}}\left(\mathcal{H}_{+}, \mathcal{H}_{-}\right):=\left\{g \in \mathrm{GL}_{\mathfrak{I}}(\mathcal{H}) \mid g^{*} V g=V\right\}$ with the Lie algebra

$$
\mathbf{L}\left(\mathrm{U}_{\mathfrak{I}}\left(\mathcal{H}_{+}, \mathcal{H}_{-}\right)\right):=\mathfrak{u}_{\mathfrak{I}}\left(\mathcal{H}_{+}, \mathcal{H}_{-}\right):=\left\{x \in \mathfrak{J} \mid x^{*} V=-V x\right\},
$$

where $\mathcal{H}=\mathcal{H}_{+} \oplus \mathcal{H}_{-}$and $V=\left(\begin{array}{cc}1 & 0 \\ 0 & -1\end{array}\right)$ with respect to this orthogonal direct sum decomposition of $\mathcal{H}$;

(BI) $\mathrm{O}_{\mathfrak{I}}\left(\mathcal{H}_{+}, \mathcal{H}_{-}\right):=\left\{g \in \mathrm{GL}_{\mathfrak{I}}(\mathcal{H}) \mid g^{-1}=J g^{*} J^{-1}\right.$ and $\left.g^{*} V g=V\right\}$ with the Lie algebra

$\mathbf{L}\left(\mathrm{O}_{\mathfrak{I}}\left(\mathcal{H}_{+}, \mathcal{H}_{-}\right)\right):=\mathfrak{o}_{\mathfrak{I}}\left(\mathcal{H}_{+}, \mathcal{H}_{-}\right):=\left\{x \in \mathfrak{J} \mid x=-J x^{*} J^{-1}\right.$ and $\left.x^{*} V=-V x\right\}$, where $\mathcal{H}=\mathcal{H}_{+} \oplus \mathcal{H}_{-}, V=\left(\begin{array}{cc}1 & 0 \\ 0 & -1\end{array}\right)$ with respect to this orthogonal direct sum decomposition of $\mathcal{H}$, and $J: \mathcal{H} \rightarrow \mathcal{H}$ is a conjugation on $\mathcal{H}$ such that $J\left(\mathcal{H}_{ \pm}\right) \subseteq \mathcal{H}_{ \pm}$

(BII) $\mathrm{O}_{\mathfrak{J}}^{*}(\mathcal{H}):=\left\{g \in \mathrm{GL}_{\mathfrak{I}}(\mathcal{H}) \mid g^{-1}=J g^{*} J^{-1}\right.$ and $\left.g \widetilde{J}=\widetilde{J} g\right\}$ with the Lie algebra

$$
\mathbf{L}\left(\mathrm{O}_{\mathfrak{J}}^{*}(\mathcal{H})\right):=\mathfrak{o}_{\mathfrak{J}}^{*}(\mathcal{H}):=\left\{x \in \mathfrak{J} \mid x=-J x^{*} J^{-1} \text { and } x \widetilde{J}=\widetilde{J} x\right\},
$$

where $J: \mathcal{H} \rightarrow \mathcal{H}$ is a conjugation and $\widetilde{J}: \mathcal{H} \rightarrow \mathcal{H}$ is an anti-conjugation such that $J \widetilde{J}=\widetilde{J} J$;

(CI) $\operatorname{Sp}_{\mathfrak{I}}(\mathcal{H} ; \mathbb{R}):=\left\{g \in \mathrm{GL}_{\mathfrak{I}}(\mathcal{H}) \mid g^{-1}=\widetilde{J} g^{*} \widetilde{J}^{-1}\right.$ and $\left.g J=J g\right\}$ with the Lie algebra

$$
\mathfrak{s p}_{\mathfrak{I}}(\mathcal{H} ; \mathbb{R}):=\left\{x \in \mathfrak{I} \mid-x=\widetilde{J} x^{*} \widetilde{J}^{-1} \text { and } x J=J x\right\},
$$

where $\widetilde{J}: \mathcal{H} \rightarrow \mathcal{H}$ is any anti-conjugation and $J: \mathcal{H} \rightarrow \mathcal{H}$ is any conjugation such that $J \widetilde{J}=\widetilde{J} J$

(CII) $\operatorname{Sp}_{\mathfrak{I}}\left(\mathcal{H}_{+}, \mathcal{H}_{-}\right):=\left\{g \in \mathrm{GL}_{\mathfrak{I}}(\mathcal{H}) \mid g^{-1}=\widetilde{J} g^{*} \widetilde{J}^{-1}\right.$ and $\left.g^{*} V g=V\right\}$ with the Lie algebra

$\mathbf{L}\left(\operatorname{Sp}_{\mathfrak{I}}\left(\mathcal{H}_{+}, \mathcal{H}_{-}\right)\right):=\mathfrak{s p}_{\mathfrak{I}}\left(\mathcal{H}_{+}, \mathcal{H}_{-}\right):=\left\{x \in \mathfrak{J} \mid x=-\widetilde{J} x^{*} \widetilde{J}^{-1}\right.$ and $\left.x^{*} V=-V x\right\}$, where $\mathcal{H}=\mathcal{H}_{+} \oplus \mathcal{H}_{-}, V=\left(\begin{array}{cc}1 & 0 \\ 0 & -1\end{array}\right)$ with respect to this orthogonal direct sum decomposition of $\mathcal{H}$, and $\widetilde{J}: \mathcal{H} \rightarrow \mathcal{H}$ is an anti-conjugation on $\mathcal{H}$ such that $\widetilde{J}\left(\mathcal{H}_{ \pm}\right) \subseteq \mathcal{H}_{ \pm}$.

We say that $\mathrm{GL}_{\mathfrak{I}}(\mathcal{H} ; \mathbb{R}), \mathrm{GL}_{\mathfrak{I}}(\mathcal{H} ; \mathbb{H}), \mathrm{U}_{\mathfrak{I}}\left(\mathcal{H}_{+}, \mathcal{H}_{-}\right), \mathrm{O}_{\mathfrak{I}}\left(\mathcal{H}_{+}, \mathcal{H}_{-}\right), \mathrm{O}_{\mathfrak{I}}^{*}(\mathcal{H}), \operatorname{Sp}_{\mathfrak{I}}(\mathcal{H} ; \mathbb{R})$, and $\operatorname{Sp}_{\mathfrak{I}}\left(\mathcal{H}_{+}, \mathcal{H}_{-}\right)$are the classical real Banach-Lie groups associated with the operator ideal $\mathfrak{I}$. Similarly, the corresponding Lie algebras are called the classical real Banach-Lie algebras (associated with $\mathfrak{I}$ ).

Remark 3.3. The classical Banach-Lie groups and algebras of Definitions 3.1 and 3.2 associated with the Schatten operator ideals $\mathfrak{S}_{p}(\mathcal{H})(1 \leq p \leq \infty)$ were introduced in Ha72, where it was conjectured that the connected 1-components of these groups have global Iwasawa decompositions in a natural sense (see subsection 8.4 in Section II.8 of Ha72]).

We also note that as a by-product of the classification of the $L^{*}$-algebras (see for instance Theorems 7.18 and 7.19 in [Be06]), every (real or complex) topologically simple $L^{*}$-algebra is isomorphic to one of the classical Banach-Lie algebras associated with the Hilbert-Schmidt ideal $\mathfrak{J}=\mathfrak{S}_{2}(\mathcal{H})$.

Problem 3.4. In the setting of Definitions 3.1 and 3.2 , the condition that $\mathfrak{I}$ should be a norm ideal is necessary in order to make the corresponding groups into smooth manifolds modeled on Banach spaces (see for instance Proposition 9.28 in Be06] or the beginning of the proof of Proposition 3.9 below). On the other hand, the 
classical "Lie" groups and Lie algebras can be defined with respect to any operator ideal, irrespective of whether it is endowed with a complete norm or not, and there exist many interesting operator ideals which do not support complete norms at all; see [KW02] and KW06].

Thus it might prove important to study the Lie-theoretic aspects of the classical groups and Lie algebras associated with arbitrary operator ideals, and perhaps to establish a bridge between the Lie theory and the commutator structure of operator ideals described in the papers [DFWW] and [We05].

We shall need the following generalization of Proposition 3 in Ba69.

Lemma 3.5. Let $\mathcal{H}$ be a complex separable infinite-dimensional Hilbert space, $\widetilde{J}: \mathcal{H} \rightarrow \mathcal{H}$ an anti-conjugation, and $\mathbb{K} \in\{\mathbb{R}, \mathbb{C}\}$. Also let $Z: \mathcal{H} \rightarrow \mathcal{H}$ be a $\mathbb{K}$ linear continuous operator such that $Z \widetilde{J}=\widetilde{J} Z$ and $Z^{2}=z_{0} \mathbf{1}$ for some $z_{0} \in(0, \infty)$. Then there exists an orthonormal basis $\left\{\xi_{l}^{(\varepsilon)}\right\}_{\substack{l \in \mathbb{Z} \backslash\{0\} \\ \varepsilon \in\left\{ \pm \sqrt{z_{0}}\right\}}}$ in the Hilbert space $\mathcal{H}$ over $\mathbb{K}$ such that $Z \xi_{ \pm l}^{(\varepsilon)}=\varepsilon \xi_{ \pm l}^{(\varepsilon)}$ and $\widetilde{J}_{ \pm l}^{(\varepsilon)}=\mp \xi_{\mp l}^{(\varepsilon)}$ whenever $\varepsilon \in\left\{ \pm \sqrt{z_{0}}\right\}$ and $l=1,2, \ldots$

Proof. Denote $\mathcal{H}^{(\varepsilon)}=\operatorname{Ker}(Z-\varepsilon)$ for $\varepsilon \in\left\{ \pm \sqrt{z_{0}}\right\}$. Since $Z^{2}=z_{0} \mathbf{1}$, it follows that $\mathcal{H}=\mathcal{H}^{\left(\sqrt{z_{0}}\right)} \oplus \mathcal{H}^{\left(-\sqrt{z_{0}}\right)}$ as an orthogonal direct sum of $\mathbb{K}$-linear closed subspaces. On the other hand $\widetilde{J} Z=Z \widetilde{J}$; hence $\widetilde{J} \mathcal{H}^{(\varepsilon)}=\mathcal{H}^{(\varepsilon)}$ whenever $\varepsilon \in\left\{ \pm \sqrt{z_{0}}\right\}$.

Now let us keep $\varepsilon \in\left\{ \pm \sqrt{z_{0}}\right\}$ fixed. We shall say that an orthonormal subset $\Sigma_{0}$ of the Hilbert space $\mathcal{H}^{(\varepsilon)}$ over $\mathbb{K}$ is a $\widetilde{J}$-set if $\{\widetilde{J} x,-\widetilde{J} x\} \cap \Sigma_{0} \neq \emptyset$ for each $x \in \Sigma_{0}$. For every $x \in \mathcal{H}^{(\varepsilon)}$ with $\|x\|=1$ we have $(x \mid \widetilde{J} x)=-\left(\widetilde{J}^{2} x \mid \widetilde{J} x\right)=-(x \mid \widetilde{J} x)$, whence $x \perp \widetilde{J} x$, so that $\{x, \widetilde{J} x\}$ is a $\widetilde{J}$-set. Then Zorn's lemma applies and shows that there exists a maximal $\widetilde{J}_{\text {-set }} \Sigma^{(\varepsilon)}$ in the Hilbert space $\mathcal{H}^{(\varepsilon)}$ over $\mathbb{K}$.

It is easy to see that $\Sigma^{(\varepsilon)}$ is actually an orthonormal basis in the Hilbert space $\mathcal{H}^{(\varepsilon)}$ over $\mathbb{K}$. In fact, let us assume that this is not the case and consider for instance the case $\mathbb{K}=\mathbb{R}$. Then there exists $x_{0} \in \mathcal{H}^{(\varepsilon)}$ such that $\left\|x_{0}\right\|=1$ and $\operatorname{Re}\left(x \mid x_{0}\right)=0$ whenever $x \in \Sigma^{(\varepsilon)}$. Now for every $x \in \Sigma^{(\varepsilon)}$ we have

$$
\operatorname{Re}\left(x \mid \widetilde{J} x_{0}\right)=-\operatorname{Re}\left(\widetilde{J}^{2} x \mid \widetilde{J} x_{0}\right)=-\operatorname{Re}\left(\widetilde{J} x \mid x_{0}\right)=0
$$

since either $\widetilde{J} x \in \Sigma^{(\varepsilon)}$ or $-\widetilde{J} x \in \Sigma^{(\varepsilon)}$. It then follows that $\Sigma^{(\varepsilon)} \cup\left\{x_{0}, \widetilde{J} x_{0}\right\}$ is again a $\widetilde{J}$-set, thus contradicting the maximality of the $\widetilde{J}_{\text {-set }} \Sigma^{(\varepsilon)}$.

Then let $\left\{\xi_{l}^{(\varepsilon)}\right\}_{l>1}$ be the set of all $x \in \Sigma^{(\varepsilon)}$ such that $-\widetilde{J} x \in \Sigma^{(\varepsilon)}$, and denote $\xi_{-l}^{(\varepsilon)}=-\widetilde{J} \xi_{l}^{(\varepsilon)}$ for $l=1,2, \ldots$. Thus we get an orthonormal basis $\left\{\xi_{l}^{(\varepsilon)}\right\}_{\substack{l \in \mathbb{Z} \backslash\{0\} \\ \varepsilon \in\left\{ \pm \sqrt{z_{0}}\right\}}}$ in the Hilbert space $\mathcal{H}$ over $\mathbb{K}$, satisfying the wished-for properties.

We shall also need the following version of Proposition 2 in Ba69].

Lemma 3.6. Let $\mathcal{H}$ be a complex separable infinite-dimensional Hilbert space with a conjugation $J: \mathcal{H} \rightarrow \mathcal{H}$. Then the following assertions hold:

(a) If $Z \in \mathcal{B}(\mathcal{H})$ satisfies the conditions $Z J=J Z, Z=Z^{*}=Z^{-1}$, and $\operatorname{dim} \operatorname{Ker}(Z-\mathbf{1})=\operatorname{dim} \operatorname{Ker}(Z+\mathbf{1})$, then there exists an orthonormal basis $\left\{\xi_{l}\right\}_{l \in \mathbb{Z} \backslash\{0\}}$ in the Hilbert space $\mathcal{H}$ such that $Z \xi_{l}=J \xi_{l}=\xi_{-l}$ whenever $l \in \mathbb{Z} \backslash\{0\}$.

(b) If $\widetilde{J}: \mathcal{H} \rightarrow \mathcal{H}$ is an anti-conjugation such that $J \widetilde{J}=\widetilde{J} J$, then there exists an orthonormal basis $\left\{\xi_{l}\right\}_{l \in \mathbb{Z} \backslash\{0\}}$ in the Hilbert space $\mathcal{H}$ such that $J \xi_{l}=\xi_{-l}$ 
whenever $l \in \mathbb{Z} \backslash\{0\}$, and $\widetilde{J} \xi_{ \pm(2 s-1)}=\xi_{\mp 2 s}$ and $\widetilde{J} \xi_{ \pm 2 s}=-\xi_{\mp(2 s-1)}$ for $s=1,2, \ldots$.

Proof. We shall denote $\mathcal{H}_{\mathbb{R}}:=\{x \in \mathcal{H} \mid J x=x\}$.

(a) Let $\mathcal{H}_{ \pm}=\operatorname{Ker}(Z \mp \mathbf{1})$. Since $V J=J V$, it follows that

$$
\mathcal{H}_{\mathbb{R}}=\left(\mathcal{H}_{\mathbb{R}} \cap \mathcal{H}_{+}\right) \oplus\left(\mathcal{H}_{\mathbb{R}} \cap \mathcal{H}_{-}\right), \quad \mathcal{H}_{ \pm}=\left(\mathcal{H}_{\mathbb{R}} \cap \mathcal{H}_{ \pm}\right) \oplus \mathrm{i}\left(\mathcal{H}_{\mathbb{R}} \cap \mathcal{H}_{ \pm}\right),
$$

and $\operatorname{dim}_{\mathbb{R}}\left(\mathcal{H}_{\mathbb{R}} \cap \mathcal{H}_{ \pm}\right)=\operatorname{dim}_{\mathbb{C}}\left(\mathcal{H}_{ \pm}\right)$(see for instance Lemma 1 in Ba69]). Then there exist countable orthonormal bases in the real Hilbert spaces $\mathcal{H}_{\mathbb{R}} \cap \mathcal{H}_{ \pm}$, which we denote by $\left\{x_{ \pm l}\right\}_{l \geq 1}$, respectively. Now define $\xi_{ \pm l}=\left(x_{l} \pm \mathrm{i} x_{-l}\right) / \sqrt{2}$ for $l=1,2, \ldots$ Then $\left\{\xi_{l}\right\}_{l \in \mathbb{Z} \backslash\{0\}}$ is the orthonormal basis in $\mathcal{H}$ which we were looking for.

(b) Since $J \widetilde{J}=\widetilde{J} J$, we can use Lemma 3.5 with $Z=J$ and $\mathbb{K}=\mathbb{R}$ to get an orthonormal basis $\left\{x_{l}^{(\varepsilon)}\right\}_{\substack{l \in \mathbb{Z} \backslash\{0\} \\ \varepsilon \in\{ \pm\}}}$ in the Hilbert space $\mathcal{H}$ over $\mathbb{R}$ such that $J x_{ \pm l}^{(\varepsilon)}=$ $\varepsilon x_{ \pm l}^{(\varepsilon)}$ and $\widetilde{J} x_{ \pm l}^{(\varepsilon)}=\mp x_{\mp l}^{(\varepsilon)}$ whenever $\varepsilon \in\{ \pm\}$ and $l=1,2, \ldots$ Let us denote $x_{l}=x_{l}^{+}$ for $l \in \mathbb{Z} \backslash\{0\}$. By using a bijection from $\{1,2, \ldots\}$ onto the set of odd integer numbers, we can re-index the sequence $\left\{x_{l}\right\}_{l \in \mathbb{Z} \backslash\{0\}}$ such that $\widetilde{J} x_{ \pm(2 s-1)}=x_{ \pm 2 s}$ and $\widetilde{J} x_{ \pm 2 s}=-x_{ \pm(2 s-1)}$ for $s=1,2, \ldots$ Now define just as above $\xi_{ \pm l}=\left(x_{l} \pm \mathrm{i} x_{-l}\right) / \sqrt{2}$ for $l=1,2, \ldots$, and thus we get an orthonormal basis in $\mathcal{H}$ satisfying the properties we wished for.

It will be convenient to have some special cases of Lemmas 3.5 and 3.6 recorded as follows. (See Propositions 2 and 3 in [Ba69] or [Nee02a].)

Lemma 3.7. Let $\mathcal{H}$ be a complex separable infinite-dimensional Hilbert space.

(a) If $J: \mathcal{H} \rightarrow \mathcal{H}$ is a conjugation, then there exists an orthonormal basis $\left\{\xi_{l}\right\}_{l \in \mathbb{Z} \backslash\{0\}}$ in $\mathcal{H}$ such that $J \xi_{l}=\xi_{-l}$ whenever $l \in \mathbb{Z} \backslash\{0\}$.

(b) If $\widetilde{J}: \mathcal{H} \rightarrow \mathcal{H}$ is an anti-conjugation, then there exists an orthonormal basis $\left\{\widetilde{\xi}_{l}\right\}_{l \in \mathbb{Z} \backslash\{0\}}$ in $\mathcal{H}$ such that $\widetilde{J} \widetilde{\xi}_{ \pm l}=\mp \xi_{\mp l}$ for $=1,2, \ldots$

Proof. Assertion (a) follows by our Lemma 3.6(a) for a suitable choice of $Z$, while assertion (b) follows by Lemma 3.5 for $Z=\mathbf{1}$.

Lemma 3.8. Let $\mathcal{H}$ be a complex separable infinite-dimensional Hilbert space with an orthonormal basis $\left\{\xi_{l}\right\}_{l \in \mathbb{Z} \backslash\{0\}}$. Assume that $\left\{\alpha_{l}\right\}_{l \in \mathbb{Z} \backslash\{0\}}$ is a bounded family of real numbers and $\varepsilon \in \mathbb{R}$, and define the self-adjoint operator

$$
A=\sum_{l \in \mathbb{Z} \backslash\{0\}} \alpha_{l}\left(\cdot \mid \xi_{l}\right) \xi_{l} \in \mathcal{B}(\mathcal{H})
$$

(where the sum is convergent in the strong operator topology). Then let $Z: \mathcal{H} \rightarrow \mathcal{H}$ be an $\mathbb{R}$-linear continuous operator such that $\|Z x\|=\|x\|$ whenever $x \in \mathcal{H}$ and satisfying either of the following conditions:

(a) $Z^{2}=1, Z \xi_{l}=\xi_{-l}$ whenever $l \in \mathbb{Z} \backslash\{0\}$, and $Z$ is either $\mathbb{C}$-linear or $\mathbb{C}$-antilinear;

(b) $Z^{2}=-1, Z \xi_{ \pm l}=\mp \xi_{\mp l}$ whenever $l=1,2, \ldots$, and $Z$ is either $\mathbb{C}$-linear or $\mathbb{C}$-antilinear.

Then we have $A=\varepsilon Z A Z^{-1}$ if and only if $\alpha_{-l}=\varepsilon \alpha_{l}$ whenever $l \in \mathbb{Z} \backslash\{0\}$. 
Proof. For every $l \in \mathbb{Z} \backslash\{0\}$ define the orthogonal projection $p_{l}=\left(\cdot \mid \xi_{l}\right) \xi_{l}$. Now assume that hypothesis (a) is satisfied and $Z$ is antilinear. Then for every vector $\xi \in \mathcal{H}$ we have

$$
\begin{aligned}
Z p_{l} Z^{-1} \xi & =Z p_{l} Z \xi=Z\left(\left(Z \xi \mid \xi_{l}\right) \xi_{l}\right)=\left(\xi_{l} \mid Z \xi\right) Z \xi_{l}=\left(Z^{2} \xi_{l} \mid Z \xi\right) Z \xi_{l} \\
& =\left(\xi \mid Z \xi_{l}\right) Z \xi_{l}=\left(\xi \mid \xi_{-l}\right) \xi_{-l}=p_{-l} \xi
\end{aligned}
$$

so that for all $l \in \mathbb{Z} \backslash\{0\}$ we have $Z p_{l} Z^{-1}=p_{-l}$. It is easy to see that the same conclusion would hold if $Z$ were linear. Thence

$$
Z A Z^{-1}=Z\left(\sum_{l \in \mathbb{Z} \backslash\{0\}} \alpha_{l} p_{l}\right) Z^{-1}=\sum_{l \in \mathbb{Z} \backslash\{0\}} \alpha_{l} p_{-l},
$$

so that the equation $Z A Z^{-1}=\varepsilon A$ is equivalent to the fact that $\alpha_{-l}=\varepsilon \alpha_{l}$ whenever $l \in \mathbb{Z} \backslash\{0\}$.

Now assume that hypothesis (b) is satisfied and $Z$ is antilinear. Then for all $l \in\{1,2, \ldots\}$ and $\xi \in \mathcal{H}$ we have

$$
\begin{aligned}
Z p_{ \pm l} Z^{-1} \xi & =-Z p_{ \pm l} Z \xi=-Z\left(\left(Z \xi \mid \xi_{ \pm l}\right) \xi_{ \pm l}\right)=-\left(\xi_{ \pm l} \mid Z \xi\right) Z \xi_{ \pm l} \\
& =\left(Z^{2} \xi_{ \pm l} \mid Z \xi\right) Z \xi_{ \pm l}=\left(\xi \mid Z \xi_{ \pm l}\right) Z \xi_{ \pm l} .
\end{aligned}
$$

Consequently $Z p_{l} Z^{-1}=p_{-l}$ for every $l \in \mathbb{Z} \backslash\{0\}$, and we would obtain the same equality if $Z$ were linear. Then the wished-for conclusion follows just as above.

Proposition 3.9. Let $\mathcal{H}$ be a complex Hilbert space and $\left\{e_{i}\right\}_{i \in I}$ a family of mutually orthogonal projections of rank 1 in $\mathcal{B}(\mathcal{H})$ such that $\sum_{i \in I} e_{i}=\mathbf{1}$. Now let $\Phi$ be a symmetric norming function, consider the corresponding Banach ideal $\mathfrak{I}=\mathfrak{S}_{\Phi}^{(0)}$, and define

$$
A:=\left\{g \in \mathrm{GL}_{\mathfrak{I}} \mid g \geq 0 ;(\forall i \in I) \quad e_{i} g=g e_{i}\right\}
$$

and

$$
\mathfrak{a}:=\left\{b \in \mathfrak{I} \mid b=b^{*} ;(\forall i \in I) \quad e_{i} b=b e_{i}\right\} .
$$

Then $A$ is a simply connected Banach-Lie subgroup of $\mathrm{GL}_{\mathfrak{I}}$ and $\mathbf{L}(A)=\mathfrak{a}$.

Proof. The proof is straightforward, using Proposition 4.4 in [Be06]. We omit the details.

\section{IWASAWA DECOMPOSITIONS FOR GROUPS OF TYPE A}

Throughout this section we let $\mathcal{H}$ be a complex separable infinite-dimensional Hilbert space, $\Phi$ a mononormalizing symmetric norming function whose Boyd indices are nontrivial, and denote the corresponding separable norm ideal by $\mathfrak{I}=$ $\mathfrak{S}_{\Phi}^{(0)} \subseteq \mathcal{B}(\mathcal{H})$. We are going to apply the abstract methods developed in Section 2 in order to construct global Iwasawa decompositions for classical groups of type A associated with the operator ideal $\mathfrak{I}$ (Definitions 3.1 and 3.2).

\section{Complex groups of type A.}

Lemma 4.1. Let $\mathcal{H}_{0}$ be a complex Hilbert space and $S=-S^{*} \in \mathcal{B}\left(\mathcal{H}_{0}\right)$ with finite spectrum, say $\sigma(S)=\left\{\lambda_{1}, \ldots, \lambda_{n}\right\}$. For $k=1, \ldots, n$ denote by $E_{k} \in \mathcal{B}\left(\mathcal{H}_{0}\right)$ the orthogonal projection onto $\operatorname{Ker}\left(S-\lambda_{k}\right)$. Then for every $Z \in \mathcal{B}\left(\mathcal{H}_{0}\right)$ we have $\int_{\mathbb{R}} \mathrm{e}^{t \cdot \operatorname{ad} S} Z \mathrm{~d} \mu(t)=\sum_{k=1}^{n} E_{k} Z E_{k}$. 
Proof. We have $S=\sum_{k=1}^{n} \lambda_{k} E_{k}$ and $E_{k} E_{l}=0$ whenever $k \neq l$; hence $\mathrm{e}^{t S}=$ $\sum_{k=1}^{n} \mathrm{e}^{t \lambda_{k}} E_{k}$ for arbitrary $t \in \mathbb{R}$. Thence

$$
\mathrm{e}^{t \cdot \operatorname{ad} S} Z=\mathrm{e}^{t S} Z \mathrm{e}^{-t S}=\sum_{k, l=1}^{n} \mathrm{e}^{t\left(\lambda_{k}-\lambda_{l}\right)} E_{k} Z E_{l} .
$$

Now $\int_{\mathbb{R}} \mathrm{e}^{t\left(\lambda_{k}-\lambda_{l}\right)} \mathrm{d} \mu(t)=\int_{\mathbb{R}} \mathrm{e}^{(t+1)\left(\lambda_{k}-\lambda_{l}\right)} \mathrm{d} \mu(t)=\mathrm{e}^{\mathrm{i}\left(\lambda_{k}-\lambda_{l}\right)} \int_{\mathbb{R}} \mathrm{e}^{t\left(\lambda_{k}-\lambda_{l}\right)} \mathrm{d} \mu(t)$ by the invariance property of $\mu$ (see Notation 2.1). If $k \neq l$, then $\lambda_{k} \neq \lambda_{l}$, whence $\int \mathrm{e}^{t\left(\lambda_{k}-\lambda_{l}\right)} \mathrm{d} \mu(t)=0$. Then the wished-for equality follows by means of equation (4.1).

Lemma 4.2. Let $\mathcal{H}$ be a separable complex Hilbert space, $\mathfrak{I}$ a separable norm ideal of $\mathcal{B}(\mathcal{H})$, and $\left\{a_{n}\right\}_{n \geq 0}$ a sequence of self-adjoint elements of $\mathcal{B}(\mathcal{H})$ which is convergent to some $a \in \mathcal{B}(\mathcal{H})$ in the strong operator topology. Then $\lim _{n \rightarrow \infty}\left\|a_{n} x-a x\right\|_{\mathfrak{J}}=$ $\lim _{n \rightarrow \infty}\left\|x a_{n}-x a\right\|_{\mathfrak{J}}=\lim _{n \rightarrow \infty}\left\|a_{n} x a_{n}-a x a\right\|_{\mathfrak{J}}=0$ whenever $x \in \stackrel{n}{\mathfrak{I}}$.

Proof. See Theorem 6.3 in Chapter III of [GK69].

In the following statement, by triangular projection associated with a self-adjoint operator we mean the triangular projection associated with its linearly ordered set of spectral projections; see [GK70, EL72], Er78], and [Pit88].

Proposition 4.3. Let $X_{0}=X_{0}^{*} \in \mathfrak{g l}_{\mathfrak{I}}$ and denote

$$
\Lambda=\left\{\lambda \in \mathbb{R} \mid \operatorname{dim} \operatorname{Ker}\left(X_{0}-\lambda\right)>0\right\},
$$

and for every $\lambda \in \Lambda$ let $E_{\lambda} \in \mathcal{B}(\mathcal{H})$ be the orthogonal projection onto $\operatorname{Ker}\left(X_{0}-\lambda\right)$.

Then $X_{0}$ is an Iwasawa quasi-regular element of $\mathfrak{g l}_{\mathfrak{I}}$ and the following assertions hold:

(1) The set $\Lambda$ is countable and $\left\{E_{\lambda}\right\}_{\lambda \in \Lambda}$ is a family of mutually orthogonal finite-rank projections satisfying $\sum_{\lambda \in \Lambda} E_{\lambda}=\mathbf{1}$ in the strong operator topology.

(2) If $\mathcal{T}_{\mathfrak{I}, X_{0}}: \mathfrak{g l}_{\mathfrak{I}} \rightarrow \mathfrak{g l}_{\mathfrak{I}}$ stands for the triangular projection associated with $X_{0}$, then

$$
\begin{aligned}
\left(\mathcal{T}_{\mathfrak{I}, X_{0}}\right)^{2} & =\mathcal{T}_{\mathfrak{I}, X_{0}}, \\
\operatorname{Ran} \mathcal{T}_{\mathfrak{I}, X_{0}} & =\left(\mathfrak{g l} \mathfrak{l}_{\mathfrak{I}}\right)_{\operatorname{ad} X_{0}}\left(\mathbb{R}_{+}\right), \\
\operatorname{Ran}\left(\mathbf{1}-\mathcal{T}_{\mathfrak{I}, X_{0}}\right) & \subseteq\left(\mathfrak{g l}_{\mathfrak{I}}\right)_{\operatorname{ad} X_{0}}\left(-\mathbb{R}_{+}\right) .
\end{aligned}
$$

(3) Assume that we write the linear operators on $\mathcal{H}$ as infinite block matrices with respect to the partition of unity given by $\left\{E_{\lambda}\right\}_{\lambda \in \Lambda}$. Then for every $X \in \mathfrak{g l}_{\mathfrak{I}}$ the matrix of $\mathcal{D}_{\mathfrak{g l}_{\mathfrak{I}}, \text { ad }\left(-\mathrm{i} X_{0}\right)} X$ can be constructed out of the matrix of $X$ by replacing all the off-diagonal blocks by zeros.

(4) If we denote $\mathfrak{a}_{\mathfrak{I}, X_{0}}=\left\{X \in \mathfrak{g l}_{\mathfrak{I}} \mid X^{*}=X\right.$ and $\left.\left[X_{0}, X\right]=0\right\}$, then

$$
\mathfrak{a}_{\mathfrak{I}, X_{0}}=\left\{X \in \mathfrak{g l}_{\mathfrak{I}} \mid X^{*}=X \text { and }(\forall \lambda \in \Lambda) \quad X E_{\lambda} \mathcal{H} \subseteq E_{\lambda} \mathcal{H}\right\} .
$$

(5) We have

$$
\left(\mathfrak{g l}_{\mathfrak{I}}\right)_{\operatorname{ad} X_{0}}\left(\mathbb{R}_{+}\right)=\left\{X \in \mathfrak{g l}_{\mathfrak{I}} \mid(\forall \lambda \in \Lambda) \quad X E_{\lambda} \mathcal{H} \subseteq \operatorname{span}\left(\bigcup_{\lambda \leq \beta \in \Lambda} E_{\beta} \mathcal{H}\right)\right\}
$$


(6) If we denote $\mathfrak{n}_{\mathfrak{I}, X_{0}}=\left(\mathfrak{g l}_{\mathfrak{I}}\right)_{\mathrm{ad}\left(-\mathrm{i} X_{0}\right)}^{0,+}$, then

$$
\mathfrak{n}_{\mathfrak{I}, X_{0}}=\left\{X \in \mathfrak{g l}_{\mathfrak{I}} \mid(\forall \lambda \in \Lambda) \quad X E_{\lambda} \mathcal{H} \subseteq \operatorname{span}\left(\bigcup_{\lambda<\beta \in \Lambda} E_{\beta} \mathcal{H}\right)\right\} .
$$

(7) The Iwasawa decomposition of $\mathfrak{g l}_{\mathfrak{I}}$ associated with $X_{0}$ is

$$
\mathfrak{g l}_{\mathfrak{I}}=\mathfrak{u}_{\mathfrak{I}} \dot{+} \mathfrak{a}_{\mathfrak{I}, X_{0}} \dot{+} \mathfrak{n}_{\mathfrak{I}, X_{0}} .
$$

(8) $X_{0}$ is an Iwasawa regular element of $\mathfrak{g l}_{\mathfrak{I}}$ if and only if it satisfies the condition

$$
(\forall \lambda \in \mathbb{R}) \quad \operatorname{dim} \operatorname{Ker}\left(X_{0}-\lambda\right) \leq 1 .
$$

Proof. The fact that $X_{0}$ is an Iwasawa quasi-regular element will follow by Proposition 2.11 as soon as we have proved assertion (2).

Assertion (1) holds true since $X_{0}$ is a compact self-adjoint operator.

We now prove assertion (2). Since the Boyd indices of the symmetric norming function $\Phi$ are nontrivial, it follows by Theorem 4.1 in Ara78 that the triangular projection defines a bounded linear idempotent operator $\mathcal{T}_{\mathfrak{I}, X_{0}}: \mathfrak{g l}_{\mathfrak{I}} \rightarrow \mathfrak{g l}_{\mathfrak{I}}$. Let $\mathfrak{F}$ denote the ideal of finite-rank operators on $\mathcal{H}$. It is clear that

$$
\mathfrak{F} \cap \operatorname{Ran} \mathcal{T}_{\mathfrak{I}, X_{0}} \subseteq\left(\mathfrak{g l}_{\mathfrak{I}}\right)_{\operatorname{ad} X_{0}}\left(\mathbb{R}_{+}\right) \quad \text { and } \quad \mathfrak{F} \cap \operatorname{Ran}\left(\mathbf{1}-\mathcal{T}_{\mathfrak{I}, X_{0}}\right) \subseteq\left(\mathfrak{g l}_{\mathfrak{I}}\right)_{\text {ad } X_{0}}\left(-\mathbb{R}_{+}\right) .
$$

Since the ideal $\mathfrak{F}$ is dense in $\mathfrak{I}\left(=\mathfrak{S}_{\Phi}^{(0)}\right)$ and $\left(\mathcal{T}_{\mathfrak{I}, X_{0}}\right)^{2}=\mathcal{T}_{\mathfrak{I}, X_{0}}$, it easily follows that $\mathfrak{F} \cap \operatorname{Ran} \mathcal{T}_{\mathfrak{I}, X_{0}}$ is dense in $\operatorname{Ran} \mathcal{T}_{\mathfrak{I}, X_{0}}$ and $\mathfrak{F} \cap \operatorname{Ran}\left(\mathbf{1}-\mathcal{T}_{\mathfrak{I}, X_{0}}\right)$ is dense in $\operatorname{Ran}\left(\mathbf{1}-\mathcal{T}_{\mathfrak{I}, X_{0}}\right)$. Thence

$$
\operatorname{Ran} \mathcal{T}_{\mathfrak{I}, X_{0}} \subseteq\left(\mathfrak{g l}_{\mathfrak{I}}\right)_{\operatorname{ad} X_{0}}\left(\mathbb{R}_{+}\right) \text {and } \operatorname{Ran}\left(\mathbf{1}-\mathcal{T}_{\mathfrak{I}, X_{0}}\right) \subseteq\left(\mathfrak{g l}_{\mathfrak{I}}\right)_{\operatorname{ad} X_{0}}\left(-\mathbb{R}_{+}\right) .
$$

Thus, to complete the proof of assertion (2), it remains to show that

$$
\operatorname{Ran} \mathcal{T}_{\mathfrak{I}, X_{0}} \supseteq\left(\mathfrak{g l}_{\mathfrak{I}}\right)_{\operatorname{ad} X_{0}}\left(\mathbb{R}_{+}\right) .
$$

To this end let $X \in\left(\mathfrak{g l}_{\mathfrak{I}}\right)_{\text {ad } X_{0}}\left(\mathbb{R}_{+}\right)$be arbitrary. Then denote by $\left\{p_{n}\right\}_{n \geq 0}$ any increasing sequence of finite-rank spectral projections of $X_{0}$ such that $\lim _{n \rightarrow \infty} p_{n}=1$ in the strong operator topology. Then Lemma 4.2 shows that $\lim _{n \rightarrow \infty}\left\|p_{n} X p_{n}-X\right\|_{\mathfrak{I}}=$ 0 ; hence it will be enough to check that $p_{n} X p_{n} \in \operatorname{Ran} \mathcal{T}_{\mathfrak{I}, X_{0}}$ whenever $n \geq 0$. For this purpose we recall that

$$
\left(\mathfrak{g l}_{\mathfrak{I}}\right)_{\operatorname{ad} X_{0}}\left(\mathbb{R}_{+}\right)=\left\{Y \in \mathfrak{g l}_{\mathfrak{I}} \mid \limsup _{t \rightarrow \infty} \frac{1}{t} \log \left\|\left(\exp \left(-t \cdot \operatorname{ad} X_{0}\right)\right) Y\right\| \leq 0\right\}
$$

(see for instance Remark 1.3 in $[\mathrm{Be} 05]$ ). Since $X \in\left(\mathfrak{g l}_{\mathfrak{I}}\right)_{\text {ad } X_{0}}\left(\mathbb{R}_{+}\right)$and for every $n \geq 0$ we have $\left(\operatorname{ad} X_{0}\right) p_{n}=0$, it then easily follows that $p_{n} X p_{n} \in\left(\mathfrak{g l}_{\mathfrak{I}}\right)_{\text {ad } X_{0}}\left(\mathbb{R}_{+}\right)$, and we have seen that this completes the proof of assertion (2).

To prove assertion (3), let $\left\{p_{n}\right\}_{n \geq 0}$ be a sequence of spectral projections of $X_{0}$ as above. For every $n \geq 0$ we have $\left(\operatorname{ad}\left(-\mathrm{i} X_{0}\right)\right) p_{n}=0$, whence it follows at once that

$$
(\forall n \geq 0) \quad p_{n}\left(\mathcal{D}_{\mathfrak{g l}_{\mathfrak{g}}, \operatorname{ad}\left(-\mathrm{i} X_{0}\right)} X\right) p_{n}=\mathcal{D}_{\mathfrak{g l}_{\mathfrak{g}}, \operatorname{ad}\left(-\mathrm{i} X_{0}\right)}\left(p_{n} X p_{n}\right) .
$$

Since $\mathcal{D}_{\mathfrak{g l}} \mathfrak{l}_{\mathfrak{I}}, \operatorname{ad}\left(-\mathrm{i} X_{0}\right): \mathfrak{g l}_{\mathfrak{I}} \rightarrow \mathfrak{g l}_{\mathfrak{I}}$ is a continuous operator and $\lim _{n \rightarrow \infty}\left\|p_{n} Y p_{n}-Y\right\|_{\mathfrak{I}}=0$ for all $Y \in \mathfrak{I}$, it then follows that it suffices to obtain the wished-for conclusion for the finite-rank operators $p_{n} X p_{n}$, where $n \geq 0$ is arbitrary, and in this (finitedimensional) case we just need to apply Lemma 4.1 .

Assertion (4) is a direct consequence of condition (4.2).

Assertion (5) follows at once by assertion (2). 
Assertion (6) follows by assertions (5) and (3).

By using assertion (2) along with Proposition 2.11 we see that $X_{0}$ is an Iwasawa quasi-regular element of $\mathfrak{g l}_{\mathfrak{I}}$ and assertion (7) holds.

Finally, we prove assertion (8): $X_{0}$ is an Iwasawa regular element if and only if $\left[\mathfrak{a}_{\mathfrak{I}, X_{0}}, \mathfrak{a}_{\mathfrak{I}, X_{0}}\right]=\{0\}$, and, by using assertion (4), we see that the latter condition is equivalent to the fact that each eigenvalue of $X_{0}$ has the spectral multiplicity equal to 1 , which is precisely condition (4.2).

We shall need the following extension of Lemma 5.2 in Chapter VI of [He01] to an infinite-dimensional setting.

Lemma 4.4. Let $U$ be a real Banach-Lie group with the Lie algebra $\mathbf{L}(U)=\mathfrak{u}$, and assume that $\mathfrak{m}$ and $\mathfrak{h}$ are two closed subalgebras of $\mathfrak{u}$ such that the direct sum decomposition $\mathfrak{u}=\mathfrak{m} \dot{+} \mathfrak{h}$ holds. Now let $M=\left\langle\exp _{U}(\mathfrak{m})\right\rangle$ and $H=\left\langle\exp _{U}(\mathfrak{h})\right\rangle$ be the corresponding subgroups of $U$ endowed with their natural structures of connected Banach-Lie groups such that $\mathbf{L}(M)=\mathfrak{m}$ and $\mathbf{L}(H)=\mathfrak{h}$. Then the multiplication map $\alpha: M \times H \rightarrow U,(m, h) \mapsto m h$, is smooth and has the property that for every $(m, h) \in M \times H$ the corresponding tangent map $T_{(m, h)}: T_{(m, h)}(M \times H) \rightarrow T_{m h} U$ is an isomorphism of Banach spaces.

Proof. The statement can be proved just as in the finite-dimensional case, so we omit the details.

Theorem 4.5. Let $X_{0}=X_{0}^{*} \in \mathfrak{g l}_{\mathfrak{I}}$ satisfying condition (4.2). Let $\Lambda=\{\lambda \in \mathbb{R} \mid$ $\left.\operatorname{dim} \operatorname{Ker}\left(X_{0}-\lambda\right)=1\right\}$, which is a linearly ordered set with respect to the reverse ordering of the real numbers, and for every $\lambda \in \Lambda$, pick $\xi_{\lambda} \in \operatorname{Ker}\left(X_{0}-\lambda\right)$ with $\left\|\xi_{\lambda}\right\|=1$. Now consider the Banach-Lie group

$$
G:=\operatorname{GL}_{\mathfrak{I}}(\mathcal{H})
$$

and its subgroups

$$
\begin{aligned}
K & :=\left\{k \in G \mid k^{*}=k^{-1}\right\}, \\
A & :=\left\{a \in G \mid(\forall \lambda \in \Lambda) \quad a \xi_{\lambda} \in \mathbb{R}_{+}^{*} \xi_{\lambda}\right\}, \text { and } \\
N & :=\left\{n \in G \mid(\forall \lambda \in \Lambda) \quad n \xi_{\lambda} \in \xi_{\lambda}+\operatorname{span}\left\{\xi_{\beta} \mid \beta<\lambda\right\}\right\} .
\end{aligned}
$$

Then $K, A$, and $N$ are Banach-Lie subgroups of $G$, and the multiplication map

$$
\boldsymbol{m}: K \times A \times N \rightarrow G, \quad(k, a, n) \mapsto k a n,
$$

is a diffeomorphism. In addition, both subgroups $A$ and $N$ are simply connected and $A N=N A$.

Proof. For every $\lambda \in \Lambda$ denote by $e_{\lambda}=\left(\cdot \mid \xi_{\lambda}\right) \xi_{\lambda}$ the orthogonal projection onto the one-dimensional subspace spanned by $\xi_{\lambda}$. Then it is easy to see that

$$
A=\left\{g \in \mathrm{GL}_{\mathfrak{I}} \mid g \geq 0 ;(\forall \lambda \in \Lambda) \quad e_{\lambda} g=g e_{\lambda}\right\} ;
$$

hence Proposition 3.9 shows that $A$ is a Banach-Lie subgroup of $G$. Furthermore, the fact that $K$ is a Banach-Lie subgroup of $G$ follows, e.g., by Proposition 9.28(ii) in $[$ Be06]. As regards $N$, let us consider the Banach algebra $\mathfrak{B}=\mathbb{C} 1+\mathfrak{I}$, and note that

$$
\begin{gathered}
N=\left\{n \in \mathfrak{B}^{\times} \mid(\forall \lambda, \beta \in I, \lambda>\beta) \quad\left(n \xi_{\lambda} \mid \xi_{\beta}\right)=0 ; \quad(\forall \lambda \in \Lambda) \quad\left(n \xi_{\lambda} \mid \xi_{\lambda}\right)=1 ;\right. \\
\left.(\forall \lambda \in \Lambda) \quad n p_{\lambda}=p_{\lambda} n p_{\lambda}\right\} ;
\end{gathered}
$$


hence $N$ is an algebraic subgroup of $\mathfrak{B}^{\times}$, and thus it is a Banach-Lie subgroup of $\mathfrak{B}^{\times}$by the Harris-Kaup theorem (see HK77] or Theorem 4.13 in Be06]). Since $N \subseteq G$ and $G$ is a Banach-Lie subgroup of $\mathfrak{B}^{\times}$, it follows that $N$ is a Banach-Lie subgroup of $G$ as well.

Now note that $\mathbf{L}(G)=\mathfrak{g l}_{\mathfrak{I}}, \mathbf{L}(K)=\mathfrak{u}_{\mathfrak{I}}, \mathbf{L}(A)=\mathfrak{a}_{\mathfrak{I}, X_{0}}$, and $\mathbf{L}(N)=\mathfrak{n}_{\mathfrak{I}, X_{0}}$ with the notation of Proposition 4.3. By using Proposition 3.9. it is easy to show that $B:=A N$ is a Banach-Lie subgroup of $G$ such that the multiplication mapping sets up a diffeomorphism $A \times N \rightarrow A N=B$. In addition, the Lie algebra of $B$ decomposes as $\mathbf{L}(B)=\mathfrak{a}_{\mathfrak{I}, X_{0}} \dot{+} \mathfrak{n}_{\mathfrak{I}, X_{0}}$. It then follows by Lemma 4.4 and Proposition 4.3 (7) that the multiplication mapping $\mathbf{m}: K \times A \times N \rightarrow G,(k, a, n) \mapsto k a n$, is regular, in the sense that its tangent map $T_{(k, a, n)} \mathbf{m}: T_{(k, a, n)}(K \times A \times N) \rightarrow T_{k a n} G$ is an isomorphism of Banach spaces for every $k \in K, a \in A$, and $n \in N$.

Now define

$$
\psi:[0,1] \times(A \times N) \rightarrow A \times N, \quad \psi(t, a, n)=((1-t) a+t \mathbf{1}, \mathbf{1}+(1-t)(n-\mathbf{1})) .
$$

Clearly $\mathbf{1}+(1-t)(n-\mathbf{1}) \in N$ whenever $n \in N$ and $t \in[0,1]$. On the other hand, for arbitrary $t \in(0,1]$ and $a \in A$ we have $(1-t) a+t \mathbf{1} \geq t \mathbf{1}$; hence $(1-t) a+t \mathbf{1}$ is a positive invertible operator; besides, $(1-t) a+t \mathbf{1}=a+t(\mathbf{1}-a) \in \mathbf{1}+\mathfrak{S}_{\Phi}^{(0)}(\mathcal{H})$, so that $(1-t) a+t \mathbf{1} \in A$. Consequently, the mapping $\psi$ is well defined. In addition, $\psi$ is continuous, $\psi(0, \cdot)=\operatorname{id}_{A \times N}$, and $\psi(1, \cdot)$ is a constant mapping of $A \times N$ into itself. Thus we see that both $A$ and $N$ are contractible topological spaces.

Since $K \cap A N=\{\mathbf{1}\}$, it is easy to see that the map $\mathbf{m}: K \times A \times N \rightarrow G$ is injective. We have seen above that the mapping $\mathbf{m}$ is regular at every point, and it then follows that $\mathbf{m}$ is a diffeomorphism of $K \times A \times N$ onto some open subset of $G$.

To prove that the multiplication mapping $\mathbf{m}$ is actually surjective, let $g \in G$ be arbitrary and consider the nest $\mathfrak{P}=\left\{p_{\lambda}\right\}_{\lambda \in \Lambda}$, where $p_{\lambda}$ is the orthogonal projection onto the subspace $\mathcal{H}_{\lambda}=\operatorname{span}\left\{\xi_{\beta} \mid \beta \leq \lambda\right\}$ of $\mathcal{H}$ whenever $\lambda \in \Lambda$. Then Corollary A.2 shows that there exist a unitary element $w \in \mathbf{1}+\mathfrak{I}$ and an element $b \in(\operatorname{Alg} \mathfrak{P})^{\times} \cap(\mathbf{1}+\mathfrak{I})$ such that $g=w b$. Now for every $\lambda \in \Lambda$ define $b_{\lambda}:=\left(b \xi_{\lambda} \mid \xi_{\lambda}\right)$. We have $\sup _{\lambda \in \Lambda}\left|b_{\lambda}\right| \leq\|v\|<\infty$, so that there exists an operator $d \in \mathcal{B}(\mathcal{H})$ such that

$$
\left(d \xi_{\lambda} \mid \xi_{\beta}\right)= \begin{cases}b_{\lambda} & \text { if } \lambda=\beta \\ 0 & \text { if } \lambda \neq \beta\end{cases}
$$

Since $b \in(\operatorname{Alg} \mathfrak{P})^{\times} \cap(\mathbf{1}+\mathfrak{I})$, it follows at once that $d \in(\operatorname{Alg} \mathfrak{P})^{\times} \cap(\mathbf{1}+\mathfrak{I})$ as well, and then $d^{-1} b \in N$. Now let $d=u|d|$ be the polar decomposition of $d$. Then $u,|d| \in G$ by Lemma 5.1 in BR05] again, so that $g=w b=w u|d|\left(d^{-1} b\right) \in K A N$, and the proof ends.

\section{Real groups of type AI.}

Theorem 4.6. Let $J: \mathcal{H} \rightarrow \mathcal{H}$ be a conjugation and $\left\{\xi_{l}\right\}_{l \in \mathbb{Z} \backslash\{0\}}$ be an orthonormal basis in $\mathcal{H}$ such that $J \xi_{l}=\xi_{l}$ whenever $l \in \mathbb{Z} \backslash\{0\}$. Pick a family of mutually different real numbers $\left\{\alpha_{l}\right\}_{l \in \mathbb{Z} \backslash\{0\}}$ such that $\lim _{l \rightarrow \pm \infty} \alpha_{l}=0$ and $\Phi\left(\alpha_{1}, \alpha_{-1}, \alpha_{2}, \alpha_{-2}, \ldots\right)<$ $\infty$, and define the self-adjoint operator

$$
X_{0}=\sum_{l \in \mathbb{Z} \backslash\{0\}} \alpha_{l}\left(\cdot \mid \xi_{l}\right) \xi_{l} \in \mathcal{B}(\mathcal{H}) .
$$


Then $X_{0}$ is an Iwasawa regular element of $\mathfrak{g l}_{\mathfrak{T}}(\mathcal{H} ; \mathbb{R})$ and the Iwasawa decomposition of $\mathfrak{g l}_{\mathfrak{I}}(\mathcal{H} ; \mathbb{R})$ associated with $X_{0}$ is

$$
\mathfrak{g l}_{\mathfrak{I}}(\mathcal{H} ; \mathbb{R})=\left(\mathfrak{u}_{\mathfrak{I}} \cap \mathfrak{g l}_{\mathfrak{I}}(\mathcal{H} ; \mathbb{R})\right) \dot{+}\left(\mathfrak{a}_{\mathfrak{J}, X_{0}} \cap \mathfrak{g l}_{\mathfrak{I}}(\mathcal{H} ; \mathbb{R})\right) \dot{+}\left(\mathfrak{n}_{\mathfrak{I}, X_{0}} \cap \mathfrak{g l}_{\mathfrak{I}}(\mathcal{H} ; \mathbb{R})\right)
$$

(where $\mathfrak{u}_{\mathfrak{I}}, \mathfrak{a}_{\mathfrak{I}, X_{0}}$, and $\mathfrak{n}_{\mathfrak{I}, X_{0}}$ are the ones defined in Proposition 4.3).

Moreover, if $G$ stands for the connected 1 -component of $\mathrm{GL}_{\mathfrak{I}}(\mathcal{H} ; \mathbb{R})$, then there exists a global Iwasawa decomposition $\boldsymbol{m}: K \times A \times N \rightarrow G$ corresponding to (4.3). In addition we have $A N=N A$, and both groups $A$ and $N$ are simply connected.

Proof. The role of the orthonormal basis $\left\{\xi_{l}\right\}_{l \in \mathbb{Z} \backslash\{0\}}$ as in the statement can be played by any orthonormal basis in the real Hilbert space $\mathcal{H}_{\mathbb{R}}=\{x \in \mathcal{H} \mid J x=x\}$. The conditions satisfied by the family $\left\{\alpha_{l}\right\}_{l \in \mathbb{Z} \backslash\{0\}}$ ensure that $X_{0}=X_{0}^{*} \in \mathfrak{I}$. In addition, it is straightforward to check that actually $X_{0} \in \mathfrak{g l}_{\mathcal{I}}(\mathcal{H}, \mathbb{R}$ ) (see for instance the proof of Lemma 3.8(a)).

On the other hand, it follows by Proposition 4.3 that $X_{0}$ is an Iwasawa quasiregular element of $\mathfrak{g l}_{\mathfrak{I}}$ and the Iwasawa decomposition of $\mathfrak{g l}_{\mathfrak{I}}$ associated with $X_{0}$ is

$$
\mathfrak{g l}_{\mathfrak{I}}=\mathfrak{u}_{\mathfrak{I}} \dot{+} \mathfrak{a}_{\mathfrak{J}, X_{0}} \dot{+} \mathfrak{n}_{\mathfrak{I}, X_{0}} \text {. }
$$

To obtain the Iwasawa decomposition asserted for $\mathfrak{g l}_{\mathfrak{I}}(\mathcal{H}, \mathbb{R})$ we are going to use Corollary 2.13 with $\widetilde{\mathfrak{g}}=\mathfrak{g l}_{\mathfrak{I}}, \mathfrak{g}_{0}=\mathfrak{g l}_{\mathfrak{I}}(\mathcal{H} ; \mathbb{R})$, and $\widetilde{\mathcal{T}}=\mathcal{T}_{\mathfrak{I}, X_{0}}: \mathfrak{g l}_{\mathfrak{I}} \rightarrow \mathfrak{g l}_{\mathfrak{I}}$ the triangular projection associated with $X_{0}$. To this end we have to prove that $\mathcal{T}_{\mathfrak{I}, X_{0}}\left(\mathfrak{g l}_{\mathfrak{I}}(\mathcal{H} ; \mathbb{R})\right) \subseteq \mathfrak{g l}_{\mathfrak{I}}(\mathcal{H} ; \mathbb{R})$.

In order to do so, we denote $\mathcal{H}_{r}=\operatorname{span}\left\{\xi_{1}, \xi_{-1}, \xi_{2}, \xi_{-2}, \ldots, \xi_{r}, \xi_{-r}\right\}$ and let $P_{r}: \mathcal{H} \rightarrow \mathcal{H}$ be the orthogonal projection onto $\mathcal{H}_{r}$ for $r=1,2, \ldots$ Then for arbitrary $X \in \mathfrak{g l}_{\mathfrak{I}}(\mathcal{H} ; \mathbb{R}) \subseteq \mathfrak{I}$ we have $\lim _{r \rightarrow \infty}\left\|P_{r} X P_{r}-X\right\|_{\mathfrak{I}}=0$ by Lemma 4.2, hence $\lim _{r \rightarrow \infty}\left\|\mathcal{T}_{\mathfrak{I}, X_{0}}\left(P_{r} X P_{r}\right)-\mathcal{T}_{\mathfrak{I}, X_{0}}(X)\right\|_{\mathfrak{I}}=0$. Thus it will be enough to show that $\mathcal{T}_{\mathfrak{J}, X_{0}}\left(P_{r} X P_{r}\right) \in \mathfrak{g l}_{\mathfrak{I}}(\mathcal{H} ; \mathbb{R})$ whenever $r \geq 1$, and this follows by the restricted root space decomposition of the finite-dimensional real reductive Lie algebras $\mathfrak{g l}\left(\mathcal{H}_{r} ; \mathbb{R}\right) \simeq \mathfrak{g l}(r, \mathbb{R})$ for $r=1,2, \ldots$.

Thus $X_{0}$ is an Iwasawa quasi-regular element of $\mathfrak{g l}_{\mathfrak{I}}(\mathcal{H} ; \mathbb{R})$. Since $X_{0}$ satisfies condition (4.2) in Proposition 4.3, it follows that it is actually Iwasawa regular in $\mathfrak{g l}_{\mathfrak{I}}$, hence also in $\mathfrak{g l}_{\mathfrak{I}}(\mathcal{H} ; \mathbb{R})$.

To obtain the global Iwasawa decomposition, let us denote by $G$ the connected 1-component of $\operatorname{GL}_{\mathfrak{I}}(\mathcal{H} ; \mathbb{R})$. We are going to apply Proposition 2.15 with $\widetilde{G}, \widetilde{K}$, $\widetilde{A}$, and $\widetilde{N}$ as in Theorem 4.5. For this purpose let us denote by $C$ the connected 1-component of $\widetilde{C} \cap \mathrm{GL}_{\mathfrak{I}}(\mathcal{H} ; \mathbb{R})$ for $C \in\{K, A, N\}$. Then $C$ will be a connected Lie subgroup of $\widetilde{G}=\mathrm{GL}_{\mathfrak{I}}$, since $\widetilde{C} \cap \mathrm{GL}_{\mathfrak{I}}(\mathcal{H} ; \mathbb{R})$ is a Lie subgroup of $\widetilde{G}$. (The latter property follows by the Harris-Kaup theorem if $C=K$ or $C=N$, and from Proposition 3.9] if $C=A$.) It is clear that $\mathbf{L}(G)=\mathfrak{g l}_{\mathfrak{I}}(\mathcal{H} ; \mathbb{R}), \mathbf{L}(K)=$ $\mathfrak{u}_{\mathfrak{I}} \cap \mathfrak{g l}_{\mathfrak{I}}(\mathcal{H} ; \mathbb{R}), \mathbf{L}(A)=\mathfrak{a}_{\mathfrak{I}, X_{0}} \cap \mathfrak{g l}_{\mathfrak{I}}(\mathcal{H} ; \mathbb{R})$, and $\mathbf{L}(N)=\mathfrak{n}_{\mathfrak{I}, X_{0}} \cap \mathfrak{g l}_{\mathfrak{I}}(\mathcal{H} ; \mathbb{R}) ;$ hence $\mathbf{L}(G)=\mathbf{L}(K) \dot{+} \mathbf{L}(A) \dot{+} \mathbf{L}(N)$. Next define $\mathcal{E}_{r}: \mathfrak{g l}_{\mathfrak{I}}(\mathcal{H} ; \mathbb{R}) \rightarrow \mathfrak{g l}_{\mathfrak{I}}(\mathcal{H} ; \mathbb{R}), X \mapsto P_{r} X P_{r}$ for $r=1,2, \ldots$ Now it is easy to see that Proposition 2.15 can be applied, and this completes the proof.

\section{Real groups of type AII.}

Theorem 4.7. Let $\widetilde{J}: \mathcal{H} \rightarrow \mathcal{H}$ be an anti-conjugation and $\left\{\widetilde{\xi}_{l}\right\}_{l \in \mathbb{Z} \backslash\{0\}}$ be an orthonormal basis in $\mathcal{H}$ such that $\widetilde{J} \widetilde{\xi}_{ \pm l}=\mp \widetilde{\xi}_{\mp l}$ for $l=1,2, \ldots$. Pick a family of real 
numbers $\left\{\alpha_{l}\right\}_{l \in \mathbb{Z} \backslash\{0\}}$ such that the numbers $\left\{\alpha_{l}\right\}_{l \geq 1}$ are mutually different and $\alpha_{-l}=\alpha_{l}$ for all $l \in \mathbb{Z} \backslash\{0\}, \quad \lim _{l \rightarrow \infty} \alpha_{l}=0, \quad$ and $\Phi\left(\alpha_{1}, \alpha_{-1}, \alpha_{2}, \alpha_{-2}, \ldots\right)<\infty$, and define the self-adjoint operator

$$
X_{0}=\sum_{l \in \mathbb{Z} \backslash\{0\}} \alpha_{l}\left(\cdot \mid \widetilde{\xi}_{l}\right) \widetilde{\xi}_{l} \in \mathcal{B}(\mathcal{H}) .
$$

Then $X_{0}$ is an Iwasawa regular element of $\mathfrak{g l}_{\mathfrak{I}}(\mathcal{H}, \mathbb{H})$ and the Iwasawa decomposition of $\mathfrak{g l}_{\mathfrak{I}}(\mathcal{H}, \mathbb{H})$ associated with $X_{0}$ is

$$
\mathfrak{g l}_{\mathfrak{I}}(\mathcal{H} ; \mathbb{H})=\left(\mathfrak{u}_{\mathfrak{I}} \cap \mathfrak{g l}_{\mathfrak{I}}(\mathcal{H} ; \mathbb{H})\right) \dot{+}\left(\mathfrak{a}_{\mathfrak{I}, X_{0}} \cap \mathfrak{g l}_{\mathfrak{I}}(\mathcal{H} ; \mathbb{H})\right) \dot{+}\left(\mathfrak{n}_{\mathfrak{I}, X_{0}} \cap \mathfrak{g l}_{\mathfrak{I}}(\mathcal{H} ; \mathbb{H})\right)
$$

(where $\mathfrak{u}_{\mathfrak{I}}, \mathfrak{a}_{\mathfrak{I}, X_{0}}$, and $\mathfrak{n}_{\mathfrak{I}, X_{0}}$ are the ones defined in Proposition 4.3). Moreover, there exists a global Iwasawa decomposition $\boldsymbol{m}: K \times A \times N \rightarrow \mathrm{GL}_{\mathfrak{I}}(\mathcal{H} ; \mathbb{H})$ corresponding to (4.4). In addition we have $A N=N A$, and both groups $A$ and $N$ are simply connected.

Proof. The orthonormal basis $\left\{\widetilde{\xi}_{l}\right\}_{l \in \mathbb{Z} \backslash\{0\}}$ as in the statement exists according to Lemma 3.7(a). The hypothesis on $\left\{\alpha_{l}\right\}_{l \in \mathbb{Z} \backslash\{0\}}$ implies that $X_{0}=X_{0}^{*} \in \mathfrak{I}$, and then $X_{0} \in \mathfrak{g l}_{\mathfrak{I}}(\mathcal{H} ; \mathbb{H})$ by Lemma $3.8(\mathrm{~b})$.

To see that $X_{0}$ is an Iwasawa quasi-regular element of $\mathfrak{g l}_{\mathfrak{I}}(\mathcal{H} ; \mathbb{H})$ and the corresponding Iwasawa decomposition looks as asserted, one can proceed just as in the proof of Theorem 4.6 this time using the orthogonal projection $\widetilde{P}_{r}: \mathcal{H} \rightarrow \mathcal{H}$ onto the subspace $\widetilde{\mathcal{H}}_{r}=\operatorname{span}\left\{\widetilde{\xi}_{1}, \widetilde{\xi}_{-1}, \widetilde{\xi}_{2}, \widetilde{\xi}_{-2}, \ldots, \widetilde{\xi}_{r}, \widetilde{\xi}_{-r}\right\}$ for $r=1,2, \ldots$ We omit the details.

It remains to check that $X_{0}$ is actually an Iwasawa regular element of $\mathfrak{g l}_{\mathfrak{I}}(\mathcal{H} ; \mathbb{H})$. To this end denote $\widetilde{\mathcal{V}}_{l}=\mathbb{C} \widetilde{\xi}_{l}+\mathbb{C} \widetilde{\xi}_{-l}$ for $l=1,2, \ldots$, and let $X=X^{*} \in \mathfrak{g l}_{\mathfrak{I}}(\mathcal{H} ; \mathbb{H})$ with $\left[X, X_{0}\right]=0$. Since the real numbers $\left\{\alpha_{l}\right\}_{l>1}$ are mutually different and $\left[X, X_{0}\right]=0$, it follows that $X \widetilde{\mathcal{V}}_{l} \subseteq \widetilde{\mathcal{V}}_{l}$ whenever $l \geq 1$. Now, since $X=X^{*}$, it follows that for each $l \geq 1$ there exists an eigenvector $v_{l} \in \widetilde{\mathcal{V}}_{l} \backslash\{0\}$ of $\left.X\right|_{\tilde{\mathcal{V}}_{l}}$. Let $\gamma_{l} \in \mathbb{R}$ be the corresponding eigenvalue, so that $X v_{l}=\gamma_{l} v_{l}$. On the other hand the anti-conjugation $\widetilde{J}$ satisfies $\widetilde{J} \widetilde{\mathcal{V}}_{l} \subseteq \widetilde{\mathcal{V}}_{l}$; hence $\widetilde{\mathcal{V}}_{l}$ has the natural structure of a quaternionic vector space. Since $\operatorname{dim}_{\mathbb{C}} \widetilde{\mathcal{V}}_{l}=2$ it follows that $\operatorname{dim}_{\mathbb{H}} \widetilde{\mathcal{V}}_{l}=1$; hence $\widetilde{\mathcal{V}}_{l}=\mathbb{H} v_{l}$. Now the operator $X \in \mathfrak{g l}_{\mathfrak{I}}(\mathcal{H} ; \mathbb{H})$ is $\mathbb{C}$-linear and $X \widetilde{J}=\widetilde{J} X$; hence for every $q \in \mathbb{H}$ we have $X\left(q v_{l}\right)=q X v_{l}=q \gamma_{l} v_{l}=\gamma_{l}\left(q v_{l}\right)$, so that $X \xi=\gamma_{l} \xi$ whenever $\xi \in \widetilde{\mathcal{V}}_{l}$.

Since $\mathcal{H}=\bigoplus_{l \geq 1} \widetilde{\mathcal{V}}_{l}$, it then follows that $\left[X_{1}, X_{2}\right]=0$ for $X_{j}=X_{j}^{*} \in \mathfrak{g l}_{\mathfrak{I}}(\mathcal{H} ; \mathbb{H})$ and $\left[X_{j}, X_{0}\right]=0$ for $j=1,2$.

To prove the assertion on the global Iwasawa decomposition one can use Proposition 2.15 in a fashion similar to that of the proof of Theorem 4.6.

\section{Real groups of type AIII.}

Theorem 4.8. Assume that $\mathcal{H}=\mathcal{H}_{+} \oplus \mathcal{H}_{-}$is an orthogonal direct sum decomposition with $\operatorname{dim} \mathcal{H}_{+}=\operatorname{dim} \mathcal{H}_{-}$, and let $\left\{e_{l}^{ \pm}\right\}_{l \geq 1}$ be an orthonormal basis in $\mathcal{H}_{ \pm}$. Then define $f_{l}^{ \pm}=\left(e_{l}^{+} \pm e_{l}^{-}\right) / \sqrt{2}$ whenever $l \geq 1$. Pick a family of real numbers $\left\{\lambda_{l}\right\}_{l \geq 1}$ such that

$$
\lambda_{j} \neq \pm \lambda_{l} \text { if } j \neq l, \quad \lim _{l \rightarrow \infty} \lambda_{l}=0, \quad \text { and } \Phi\left(\lambda_{1},-\lambda_{1}, \lambda_{2},-\lambda_{2}, \ldots\right)<\infty
$$


and define the self-adjoint operator

$$
X_{0}:=\sum_{l \geq 1} \lambda_{l}\left(\left(\cdot \mid f_{l}^{+}\right) f_{l}^{+}-\left(\cdot \mid f_{l}^{-}\right) f_{l}^{-}\right) .
$$

Then $X_{0}$ is an Iwasawa regular element of $\mathfrak{u}_{\mathfrak{I}}\left(\mathcal{H}_{+}, \mathcal{H}_{-}\right)$and the corresponding Iwasawa decomposition is

$$
\begin{aligned}
\mathfrak{u}_{\mathfrak{I}}\left(\mathcal{H}_{+}, \mathcal{H}_{-}\right)= & \left(\mathfrak{u}_{\mathfrak{I}} \cap \mathfrak{u}_{\mathfrak{I}}\left(\mathcal{H}_{+}, \mathcal{H}_{-}\right)\right) \dot{+}\left(\mathfrak{a}_{\mathfrak{I}, X_{0}} \cap \mathfrak{u}_{\mathfrak{I}}\left(\mathcal{H}_{+}, \mathcal{H}_{-}\right)\right) \\
& \dot{+}\left(\mathfrak{n}_{\mathfrak{I}, X_{0}} \cap \mathfrak{u}_{\mathfrak{I}}\left(\mathcal{H}_{+}, \mathcal{H}_{-}\right)\right)
\end{aligned}
$$

(where $\mathfrak{u}_{\mathfrak{I}}, \mathfrak{a}_{\mathfrak{I}, X_{0}}$, and $\mathfrak{n}_{\mathfrak{I}, X_{0}}$ are the ones defined in Proposition 4.3).

Moreover, if $G$ stands for the connected 1-component of $\mathrm{U}_{\mathfrak{I}}\left(\mathcal{H}_{+}, \mathcal{H}_{-}\right)$, then there exists a global Iwasawa decomposition $\boldsymbol{m}: K \times A \times N \rightarrow G$ corresponding to (4.3). In addition we have $A N=N A$, and both groups $A$ and $N$ are simply connected.

Proof. To begin with, note that $\bigcup_{l \geq 1}\left\{f_{l}^{+}, f_{l}^{-}\right\}$is an orthonormal basis in $\mathcal{H}$. It then follows by the hypothesis on $\left\{\bar{\lambda}_{l}\right\}_{l \geq 1}$ along with Proposition 4.3 that $X_{0}$ is an Iwasawa regular element of $\mathfrak{g l}_{\mathfrak{I}}$. We now show that actually $X_{0} \in \mathfrak{u}_{\mathfrak{I}}\left(\mathcal{H}_{+}, \mathcal{H}_{-}\right)$. For this purpose denote $V=\left(\begin{array}{cc}1 & 0 \\ 0 & -1\end{array}\right)$ as in Definition 3.2. Then for all $j \geq 1$ we have $V e_{j}^{ \pm}= \pm e_{j}^{ \pm}$, whence $V f_{j}^{ \pm}=f_{j}^{\mp}$. This implies that for every $\xi \in \mathcal{H}$ we have

$$
\left(\xi \mid f_{j}^{ \pm}\right) f_{j}^{ \pm}=\left(\xi \mid V f_{j}^{\mp}\right) f_{j}^{ \pm}=V\left(\left(V \xi \mid f_{j}^{\mp}\right) f_{j}^{\mp}\right) .
$$

Thus $X_{0}=-V X_{0} V$, whence $X_{0} V=-V X_{0}$, and then $X_{0} \in \mathfrak{u}_{\mathfrak{I}}\left(\mathcal{H}_{+}, \mathcal{H}_{-}\right)$.

Now the wished-for conclusion will follow by Corollary 2.13 as soon as we have proved that the triangular projection $\mathcal{T}_{\mathfrak{I}, X_{0}}: \mathfrak{I} \rightarrow \mathfrak{I}$ leaves $\mathfrak{u}_{\mathfrak{I}}\left(\mathcal{H}_{+}, \mathcal{H}_{-}\right)$invariant. To this end, for $r=1,2, \ldots$ denote $\mathcal{H}_{r}=\operatorname{span}\left\{e_{1}^{+}, e_{1}^{-}, e_{2}^{+}, e_{2}^{-}, \ldots, e_{r}^{+}, e_{r}^{-}\right\}$and let $P_{r}: \mathcal{H} \rightarrow \mathcal{H}$ be the orthogonal projection onto $\mathcal{H}_{r}$. For $X \in \mathfrak{u}_{\mathfrak{I}}\left(\mathcal{H}_{+}, \mathcal{H}_{-}\right) \subseteq \mathfrak{I}$ we have $\lim _{r \rightarrow \infty}\left\|P_{r} X P_{r}-X\right\|_{\mathfrak{I}}=0$ by Lemma 4.2, hence

$$
\lim _{r \rightarrow \infty}\left\|\mathcal{T}_{\mathfrak{I}, X_{0}}\left(P_{r} X P_{r}\right)-\mathcal{T}_{\mathfrak{I}, X_{0}}(X)\right\|_{\mathfrak{I}}=0 .
$$

Thus it will be enough to show that $\mathcal{T}_{\mathfrak{I}, X_{0}}\left(P_{r} X P_{r}\right) \in \mathfrak{u}_{\mathfrak{I}}\left(\mathcal{H}_{+}, \mathcal{H}_{-}\right)$whenever $r \geq 1$, and this follows by the restricted-root space decomposition of the finite-dimensional real reductive Lie algebras $\mathfrak{u}\left(\mathcal{H}_{r} \cap \mathcal{H}_{+}, \mathcal{H}_{r} \cap \mathcal{H}_{-}\right) \simeq \mathfrak{u}(r, r)$ for $r=1,2, \ldots$

To prove the assertion on the global Iwasawa decomposition one can use Proposition 2.15 in a fashion similar to that of the proof of Theorem 4.6.

\section{IWASAWA DECOMPOSITIONS FOR GROUPS OF TYPE B}

As in Section 4 we let $\mathcal{H}$ be a complex separable infinite-dimensional Hilbert space, $\Phi$ a mononormalizing symmetric norming function whose Boyd indices are nontrivial, and let $\mathfrak{I}=\mathfrak{S}_{\Phi}^{(0)} \subseteq \mathcal{B}(\mathcal{H})$ denote the corresponding separable norm ideal. We shall use the methods of Section 2 to get global Iwasawa decompositions for classical groups of type B associated with the operator ideal $\mathfrak{I}$.

\section{Complex groups of type B.}

Theorem 5.1. Let $J: \mathcal{H} \rightarrow \mathcal{H}$ be a conjugation and $\left\{\xi_{l}\right\}_{l \in \mathbb{Z} \backslash\{0\}}$ be an orthonormal basis in $\mathcal{H}$ such that $J \xi_{l}=\xi_{-l}$ whenever $l \in \mathbb{Z} \backslash\{0\}$. Pick a family of mutually different real numbers $\left\{\alpha_{l}\right\}_{l \in \mathbb{Z} \backslash\{0\}}$ satisfying the conditions

$\alpha_{-l}=-\alpha_{l}$ for all $l \in \mathbb{Z} \backslash\{0\}, \quad \lim _{l \rightarrow \infty} \alpha_{l}=0, \quad$ and $\Phi\left(\alpha_{1}, \alpha_{-1}, \alpha_{2}, \alpha_{-2}, \ldots\right)<\infty$, 
and define the self-adjoint operator

$$
X_{0}=\sum_{l \in \mathbb{Z} \backslash\{0\}} \alpha_{l}\left(\cdot \mid \xi_{l}\right) \xi_{l} \in \mathcal{B}(\mathcal{H}) .
$$

Then $X_{0}$ is an Iwasawa regular element of $\mathfrak{o}_{\mathfrak{I}}$ and the Iwasawa decomposition of $\mathfrak{o}_{\mathfrak{I}}$ associated with $X_{0}$ is

$$
\mathfrak{o}_{\mathfrak{I}}=\left(\mathfrak{u}_{\mathfrak{I}} \cap \mathfrak{o}_{\mathfrak{I}}\right) \dot{+}\left(\mathfrak{a}_{\mathfrak{I}, X_{0}} \cap \mathfrak{o}_{\mathfrak{I}}\right) \dot{+}\left(\mathfrak{n}_{\mathfrak{I}, X_{0}} \cap \mathfrak{o}_{\mathfrak{I}}\right)
$$

(where $\mathfrak{u}_{\mathfrak{I}}, \mathfrak{a}_{\mathfrak{I}, X_{0}}$, and $\mathfrak{n}_{\mathfrak{I}, X_{0}}$ are the ones defined in Proposition 4.3).

Moreover, if $G$ stands for the connected 1-component of $\mathrm{O}_{\mathfrak{I}}$, then there exists a global Iwasawa decomposition $\boldsymbol{m}: K \times A \times N \rightarrow G$ corresponding to (5.1). In addition we have $A N=N A$, and both groups $A$ and $N$ are simply connected.

Proof. Recall that the existence of the orthonormal basis $\left\{\xi_{l}\right\}_{l \in \mathbb{Z} \backslash\{0\}}$ as in the statement follows by Lemma $3.7(\mathrm{a})$. The conditions satisfied by the family $\left\{\alpha_{l}\right\}_{l \in \mathbb{Z} \backslash\{0\}}$ ensure that $X_{0}=X_{0}^{*} \in \mathfrak{I}$. In addition, it follows by Lemma 3.8(a) that actually $X_{0} \in \mathfrak{o}_{\mathfrak{I}}$.

On the other hand, since the real numbers in the family $\left\{\alpha_{l}\right\}_{l \in \mathbb{Z} \backslash\{0\}}$ are mutually different, it follows by Proposition 4.3 that $X_{0}$ is an Iwasawa regular element of $\mathfrak{g l}_{\mathfrak{I}}$ and the Iwasawa decomposition of $\mathfrak{g l}_{\mathfrak{I}}$ associated with $X_{0}$ is $\mathfrak{g l}_{\mathfrak{I}}=\mathfrak{u}_{\mathfrak{I}} \dot{+} \mathfrak{a}_{\mathfrak{I}, X_{0}} \dot{+} \mathfrak{n}_{\mathfrak{I}, X_{0}}$. To obtain the conclusion we are going to use Corollary 2.12 for $\widetilde{\mathfrak{g}}=\mathfrak{g l}_{\mathfrak{I}}, \mathfrak{g}=\mathfrak{o}_{\mathfrak{I}}$, and $\widetilde{\mathcal{T}}=\mathcal{T}_{\mathfrak{I}, X_{0}}: \mathfrak{g l}_{\mathfrak{I}} \rightarrow \mathfrak{g l}_{\mathfrak{I}}$. To this end it remains to prove that $\mathcal{T}_{\mathfrak{I}, X_{0}}\left(\mathfrak{o}_{\mathfrak{I}}\right) \subseteq \mathfrak{o}_{\mathfrak{I}}$.

Denote $\mathcal{H}_{r}=\operatorname{span}\left\{\xi_{1}, \xi_{-1}, \xi_{2}, \xi_{-2}, \ldots, \xi_{r}, \xi_{-r}\right\}$ for $r \geq 1$. Also let $P_{r}: \mathcal{H} \rightarrow \mathcal{H}$ be the orthogonal projection onto $\mathcal{H}_{r}$ for $r=1,2, \ldots$ Then for arbitrary $X \in \mathfrak{o}_{\mathfrak{I}} \subseteq \mathfrak{I}$ we have $\lim _{r \rightarrow \infty}\left\|P_{r} X P_{r}-X\right\|_{\mathfrak{I}}=0$ by Lemma 4.2, hence

$$
\lim _{r \rightarrow \infty}\left\|\mathcal{T}_{\mathfrak{I}, X_{0}}\left(P_{r} X P_{r}\right)-\mathcal{T}_{\mathfrak{I}, X_{0}}(X)\right\|_{\mathfrak{I}}=0
$$

Thus it will be enough to show that $\mathcal{T}_{\mathfrak{I}, X_{0}}\left(P_{r} X P_{r}\right) \in \mathfrak{o}_{\mathfrak{I}}$ whenever $r \geq 1$, and this follows by the restricted-root space decomposition of the finite-dimensional complex reductive Lie algebras $\mathfrak{o}\left(\mathcal{H}_{r}\right) \simeq \mathfrak{o}(r, \mathbb{C})$ for $r=1,2, \ldots$

To prove the assertion on the global Iwasawa decomposition one can use Proposition 2.15 in a fashion similar to that of the proof of Theorem 4.6.

\section{Real groups of type BI.}

Theorem 5.2. Assume $\mathcal{H}=\mathcal{H}_{+} \oplus \mathcal{H}_{-}$with $\operatorname{dim} \mathcal{H}_{+}=\operatorname{dim} \mathcal{H}_{-}$and let $J: \mathcal{H} \rightarrow \mathcal{H}$ be a conjugation such that $J\left(\mathcal{H}_{ \pm}\right) \subseteq \mathcal{H}_{ \pm}$. Also let $V=\left(\begin{array}{cc}1 & 0 \\ 0 & -1\end{array}\right)$ with respect to this orthogonal direct sum decomposition of $\mathcal{H}$. Then let $\left\{\xi_{l}\right\}_{l \in \mathbb{Z} \backslash\{0\}}$ be an orthonormal basis in $\mathcal{H}$ such that $J \xi_{l}=V \xi_{l}=\xi_{-l}$ whenever $l \in \mathbb{Z} \backslash\{0\}$. Pick a family of mutually different real numbers $\left\{\alpha_{l}\right\}_{l \in \mathbb{Z} \backslash\{0\}}$ satisfying the conditions

$\alpha_{-l}=-\alpha_{l}$ for all $l \in \mathbb{Z} \backslash\{0\}, \quad \lim _{l \rightarrow \infty} \alpha_{l}=0, \quad$ and $\Phi\left(\alpha_{1}, \alpha_{-1}, \alpha_{2}, \alpha_{-2}, \ldots\right)<\infty$, and define the self-adjoint operator

$$
X_{0}=\sum_{l \in \mathbb{Z} \backslash\{0\}} \alpha_{l}\left(\cdot \mid \xi_{l}\right) \xi_{l} \in \mathcal{B}(\mathcal{H}) .
$$


Then $X_{0}$ is an Iwasawa regular element of $\mathfrak{o}_{\mathfrak{I}}\left(\mathcal{H}_{+}, \mathcal{H}_{-}\right)$and the corresponding Iwasawa decomposition is

$$
\begin{aligned}
\mathfrak{o}_{\mathfrak{I}}\left(\mathcal{H}_{+}, \mathcal{H}_{-}\right)= & \left(\mathfrak{u}_{\mathfrak{I}} \cap \mathfrak{o}_{\mathfrak{I}}\left(\mathcal{H}_{+}, \mathcal{H}_{-}\right)\right) \dot{+}\left(\mathfrak{a}_{\mathfrak{I}, X_{0}} \cap \mathfrak{o}_{\mathfrak{I}}\left(\mathcal{H}_{+}, \mathcal{H}_{-}\right)\right) \\
& \dot{+}\left(\mathfrak{n}_{\mathfrak{I}, X_{0}} \cap \mathfrak{o}_{\mathfrak{I}}\left(\mathcal{H}_{+}, \mathcal{H}_{-}\right)\right)
\end{aligned}
$$

(where $\mathfrak{u}_{\mathfrak{I}}, \mathfrak{a}_{\mathfrak{I}, X_{0}}$, and $\mathfrak{n}_{\mathfrak{I}, X_{0}}$ are the ones defined in Proposition 4.3).

Moreover if $G$ stands for the connected 1-component of $\mathrm{O}_{\mathfrak{I}}\left(\mathcal{H}_{+}, \mathcal{H}_{-}\right)$, then there exists a global Iwasawa decomposition $\boldsymbol{m}: K \times A \times N \rightarrow G$ corresponding to (5.2). In addition we have $A N=N A$, and both groups $A$ and $N$ are simply connected.

Proof. The existence of the orthonormal basis $\left\{\xi_{l}\right\}_{l \in \mathbb{Z} \backslash\{0\}}$ as in the statement follows by Lemma 3.6. The conditions satisfied by the family of real numbers $\left\{\alpha_{l}\right\}_{l \in \mathbb{Z} \backslash\{0\}}$ ensure that $X_{0}=X_{0}^{*} \in \mathfrak{I}$. In addition, it follows by Lemma 3.8 (a) that we actually have $X_{0} \in \mathfrak{o}_{\mathfrak{I}}\left(\mathcal{H}_{+}, \mathcal{H}_{-}\right)$.

On the other hand, since the real numbers in the family $\left\{\alpha_{l}\right\}_{l \in \mathbb{Z} \backslash\{0\}}$ are mutually different, it follows by Proposition 4.3 that $X_{0}$ is an Iwasawa regular element of $\mathfrak{g l}_{\mathfrak{I}}$ and the corresponding Iwasawa decomposition is $\mathfrak{g l}_{\mathfrak{I}}=\mathfrak{u}_{\mathfrak{I}} \dot{+} \mathfrak{a}_{\mathfrak{I}, X_{0}} \dot{+} \mathfrak{n}_{\mathfrak{I}, X_{0}}$. Thus the conclusion will follow by applying Corollary 2.13 for the data $\widetilde{\mathfrak{g}}=\mathfrak{g l}_{\mathfrak{I}}$, $\mathfrak{g}_{0}=\mathfrak{o}_{\mathfrak{I}}\left(\mathcal{H}_{+}, \mathcal{H}_{-}\right)$, and $\widetilde{\mathcal{T}}=\mathcal{T}_{\mathfrak{I}, X_{0}}: \mathfrak{g l}_{\mathfrak{I}} \rightarrow \mathfrak{g l}_{\mathfrak{I}}$. To this end it only remains to prove that $\mathcal{T}_{\mathfrak{I}, X_{0}}\left(\mathfrak{o}_{\mathfrak{I}}\left(\mathcal{H}_{+}, \mathcal{H}_{-}\right)\right) \subseteq \mathfrak{o}_{\mathfrak{I}}\left(\mathcal{H}_{+}, \mathcal{H}_{-}\right)$. In order to do so, we denote

$$
\mathcal{H}_{r}=\operatorname{span}\left\{\xi_{1}, \xi_{-1}, \xi_{2}, \xi_{-2}, \ldots, \xi_{r}, \xi_{-r}\right\} \text { whenever } r \geq 1 .
$$

Also let $P_{r}: \mathcal{H} \rightarrow \mathcal{H}$ be the orthogonal projection onto $\mathcal{H}_{r}$ for $r=1,2, \ldots$ Then for arbitrary $X \in \mathfrak{o}_{\mathfrak{I}} \subseteq \mathfrak{I}$ we have $\lim _{r \rightarrow \infty}\left\|P_{r} X P_{r}-X\right\|_{\mathfrak{I}}=0$ by Lemma 4.2 hence

$$
\lim _{r \rightarrow \infty}\left\|\mathcal{T}_{\mathfrak{I}, X_{0}}\left(P_{r} X P_{r}\right)-\mathcal{T}_{\mathfrak{I}, X_{0}}(X)\right\|_{\mathfrak{I}}=0 .
$$

Thus it will be enough to show that $\mathcal{T}_{\mathfrak{I}, X_{0}}\left(P_{r} X P_{r}\right) \in \mathfrak{o}_{\mathfrak{I}}\left(\mathcal{H}_{+}, \mathcal{H}_{-}\right)$whenever $r \geq 1$, and this follows by the restricted-root space decomposition of the finite-dimensional real reductive Lie algebras $\mathfrak{o}\left(\mathcal{H}_{r} \cap \mathcal{H}_{+}, \mathcal{H}_{r} \cap \mathcal{H}_{-}\right) \simeq \mathfrak{o}(r, r)$ for $r=1,2, \ldots$

To prove the assertion on the global Iwasawa decomposition one can use Proposition 2.15 in a fashion similar to that of the proof of Theorem 4.6.

\section{Real groups of type BII.}

Theorem 5.3. Let $J: \mathcal{H} \rightarrow \mathcal{H}$ be a conjugation and $\widetilde{J}: \mathcal{H} \rightarrow \mathcal{H}$ be an anticonjugation such that $J \widetilde{J}=\widetilde{J} J$. Then let $\left\{\xi_{l}\right\}_{l \in \mathbb{Z} \backslash\{0\}}$ be an orthonormal basis in the Hilbert space $\mathcal{H}$ such that $J \xi_{l}=\xi_{-l}$ whenever $l \in \mathbb{Z} \backslash\{0\}$, and $\widetilde{J} \xi_{ \pm(2 s-1)}=\xi_{\mp 2 s}$ and $\widetilde{J} \xi_{ \pm 2 s}=-\xi_{\mp(2 s-1)}$ for $s=1,2, \ldots$

Pick a family of real numbers $\left\{\alpha_{l}\right\}_{l \in \mathbb{Z} \backslash\{0\}}$ such that $\alpha_{-l}=-\alpha_{l}$ for all $l \in \mathbb{Z} \backslash\{0\}$, $\alpha_{2 s-1} \neq \pm \alpha_{2 t-1}$ whenever $s \neq t$ and $s, t \geq 1, \alpha_{2 s-1}=-\alpha_{2 s}$ for $s=1,2, \ldots$, $\lim _{l \rightarrow \infty} \alpha_{l}=0$, and $\Phi\left(\alpha_{1}, \alpha_{-1}, \alpha_{2}, \alpha_{-2}, \ldots\right)<\infty$, and define the self-adjoint operator

$$
X_{0}=\sum_{l \in \mathbb{Z} \backslash\{0\}} \alpha_{l}\left(\cdot \mid \xi_{l}\right) \xi_{l} \in \mathcal{B}(\mathcal{H}) .
$$

Then $X_{0}$ is an Iwasawa regular element of $\mathfrak{o}_{\mathfrak{J}}^{*}(\mathcal{H})$ and the corresponding Iwasawa decomposition is

$$
\mathfrak{o}_{\mathfrak{J}}^{*}(\mathcal{H})=\left(\mathfrak{u}_{\mathfrak{J}} \cap \mathfrak{o}_{\mathfrak{J}}^{*}(\mathcal{H})\right) \dot{+}\left(\mathfrak{a}_{\mathfrak{I}, X_{0}} \cap \mathfrak{o}_{\mathfrak{J}}^{*}(\mathcal{H})\right) \dot{+}\left(\mathfrak{n}_{\mathfrak{I}, X_{0}} \cap \mathfrak{o}_{\mathfrak{J}}^{*}(\mathcal{H})\right)
$$

(where $\mathfrak{u}_{\mathfrak{I}}, \mathfrak{a}_{\mathfrak{I}, X_{0}}$, and $\mathfrak{n}_{\mathfrak{I}, X_{0}}$ are the ones defined in Proposition 4.3). 
Moreover, if $G$ stands for the connected 1 -component of $\mathrm{O}_{\mathfrak{J}}^{*}(\mathcal{H})$, then there exists a global Iwasawa decomposition $\boldsymbol{m}: K \times A \times N \rightarrow G$ corresponding to (5.3). In addition we have $A N=N A$, and both groups $A$ and $N$ are simply connected.

Proof. The existence of the orthonormal basis $\left\{\xi_{l}\right\}_{l \in \mathbb{Z} \backslash\{0\}}$ as in the statement follows by Lemma 3.6. Moreover, by Lemma 3.8 we have $X_{0} \in \mathfrak{o}_{\mathfrak{J}}$. On the other hand, since $X_{0}=\sum_{l \geq 1} \alpha_{l}\left(\left(\cdot \mid \xi_{l}\right) \xi_{l}-\left(\cdot \mid \xi_{-l}\right) \xi_{-l}\right)$, it follows (see the proof of Lemma 3.8(b)) that

$$
\widetilde{J} X_{0} \widetilde{J}^{-1}=\sum_{l \geq 1} \alpha_{l}\left(\left(\cdot \mid \widetilde{J} \xi_{l}\right) \widetilde{J} \xi_{l}-\left(\cdot \mid \widetilde{J} \xi_{-l}\right) \widetilde{J} \xi_{-l}\right)
$$

Now, since $\widetilde{J} \xi_{ \pm(2 s-1)}=\xi_{\mp 2 s}, \widetilde{J} \xi_{ \pm 2 s}=-\xi_{\mp(2 s-1)}$, and $\alpha_{2 s-1}=-\alpha_{2 s}$ whenever $s=1,2, \ldots$, we see that $\widetilde{J} X_{0} \widetilde{J}^{-1}=X_{0}$. Thus $X_{0} \in \mathfrak{g l}_{\mathfrak{I}}(\mathcal{H} ; \mathbb{H}) \cap \mathfrak{o}_{\mathfrak{I}}=\mathfrak{o}_{\mathfrak{I}}^{*}(\mathcal{H})$.

By using the projections onto the subspaces

$$
\mathcal{H}_{s}=\operatorname{span}\left\{\xi_{1}, \xi_{-1}, \xi_{2}, \xi_{-2}, \ldots, \xi_{2 s}, \xi_{-2 s}\right\}
$$

and Corollary 2.13 as in the proof of Proposition 5.2. it then follows that $X_{0}$ is an Iwasawa quasi-regular element of $\mathfrak{o}_{\mathfrak{J}}^{*}(\mathcal{H})$ and the corresponding Iwasawa decomposition looks as asserted. It remains to prove that $X_{0}$ is actually an Iwasawa regular element of $\mathfrak{o}_{\mathfrak{J}}^{*}(\mathcal{H})$, and this fact can be obtained as in the proof of Proposition 4.7. Specifically, denote $\mathcal{V}_{s}^{\prime}=\mathbb{C} \xi_{2 s-1}+\mathbb{C} \xi_{-2 s}, \mathcal{V}_{s}^{\prime \prime}=\mathbb{C} \xi_{-(2 s-1)}+\mathbb{C} \xi_{2 s}$, and $\mathcal{V}_{s}=\mathcal{V}_{s}^{\prime} \oplus \mathcal{V}_{s}^{\prime \prime}$ for $s=1,2, \ldots$ Let $X=X^{*} \in \mathfrak{o}_{\mathfrak{J}}^{*}$ such that $\left[X, X_{0}\right]=0$. Since $\alpha_{2 s-1} \neq \pm \alpha_{2 t-1}$ whenever $s \neq t$ and $s, t \geq 1$, it follows that $X$ leaves each of the subspaces $\mathcal{V}_{s}^{\prime}$ and $\mathcal{V}_{s}^{\prime \prime}$ invariant for $s=1,2, \ldots$. Since $X=X^{*}$, it follows that $X$ has eigenvectors $v_{s}^{\prime} \in \widetilde{\mathcal{V}}_{s}^{\prime} \backslash\{0\}$ and $v_{s}^{\prime \prime} \in \widetilde{\mathcal{V}}_{s}^{\prime \prime} \backslash\{0\}$. Let $\gamma_{s}^{\prime}, \gamma_{s}^{\prime \prime} \in \mathbb{R}$ be the corresponding eigenvalues, so that $X v_{s}^{\prime}=\gamma_{s}^{\prime} v_{s}^{\prime}$ and $X v_{s}^{\prime \prime}=\gamma_{s}^{\prime \prime} v_{s}^{\prime \prime}$. On the other hand the anti-conjugation $\widetilde{J}$ satisfies $\widetilde{J} \widetilde{\mathcal{V}}_{s}^{\prime} \subseteq \widetilde{\mathcal{V}}_{s}^{\prime}$ and $\widetilde{\mathcal{J}} \widetilde{\mathcal{V}}_{s}^{\prime \prime} \subseteq \widetilde{\mathcal{V}}_{s}^{\prime \prime}$; hence both $\mathcal{V}_{s}^{\prime}$ and $\mathcal{V}_{s}^{\prime \prime}$ have the natural structures of a quaternionic vector space. By counting dimensions, we get $\mathcal{V}_{s}^{\prime}=\mathbb{H} v_{s}^{\prime}$ and $\mathcal{V}_{s}^{\prime \prime}=\mathbb{H} v_{s}^{\prime \prime}$. The operator $X \in \mathfrak{g l}_{\mathfrak{T}}(\mathcal{H} ; \mathbb{H})$ is $\mathbb{C}$-linear and $X \widetilde{J}=\widetilde{J} X$; hence for every $q \in \mathbb{H}$ we have $X\left(q v_{s}^{\prime}\right)=q X v_{s}^{\prime}=\gamma_{s}^{\prime}\left(q v_{s}^{\prime}\right)$, so that $X \xi=\gamma_{s}^{\prime} \xi$ whenever $\xi \in \mathcal{V}_{s}^{\prime}$. Similarly $X \xi=\gamma_{s}^{\prime \prime} \xi$ whenever $\xi \in \mathcal{V}_{s}^{\prime \prime}$. Since $\mathcal{H}=\bigoplus_{s \geq 1}\left(\mathcal{V}_{s}^{\prime} \oplus \mathcal{V}_{s}^{\prime \prime}\right)$, it then follows that $\left[X_{1}, X_{2}\right]=0$ whenever $X_{j}=X_{j}^{*} \in \mathfrak{o}_{\mathfrak{J}}^{*}(\mathcal{H})$ and $\left[X_{j}, X_{0}\right]=0$ for $j=1,2$.

To prove the assertion on the global Iwasawa decomposition one can use Proposition 2.15 in a fashion similar to that of the proof of Theorem 4.6.

\section{IWASAWA DECOMPOSITIONS FOR GROUPS OF TYPE C}

Just as in Sections 4 and 5 we let $\mathcal{H}$ be a complex separable infinite-dimensional Hilbert space, $\Phi$ a mononormalizing symmetric norming function whose Boyd indices are nontrivial, and denote the corresponding separable norm ideal by $\mathfrak{I}=$ $\mathfrak{S}_{\Phi}^{(0)} \subseteq \mathcal{B}(\mathcal{H})$. As above, we shall use the methods of Section 2 to get global Iwasawa decompositions for classical groups of type $\mathrm{C}$ associated with the operator ideal $\mathfrak{I}$.

\section{Complex groups of type C.}

Theorem 6.1. Let $\widetilde{J}: \mathcal{H} \rightarrow \mathcal{H}$ be an anti-conjugation and $\left\{\widetilde{\xi}_{l}\right\}_{l \in \mathbb{Z} \backslash\{0\}}$ be an orthonormal basis in $\mathcal{H}$ such that $\widetilde{J} \widetilde{\xi}_{ \pm l}=\mp \widetilde{\xi}_{\mp l}$ for $l=1,2, \ldots$ Now pick a family of 
mutually different real numbers $\left\{\alpha_{l}\right\}_{l \in \mathbb{Z} \backslash\{0\}}$ satisfying the conditions $\alpha_{-l}=-\alpha_{l}$ for all $l \in \mathbb{Z} \backslash\{0\}, \quad \lim _{l \rightarrow \infty} \alpha_{l}=0, \quad$ and $\Phi\left(\alpha_{1}, \alpha_{-1}, \alpha_{2}, \alpha_{-2}, \ldots\right)<\infty$, and define the self-adjoint operator

$$
X_{0}=\sum_{l \in \mathbb{Z} \backslash\{0\}} \alpha_{l}\left(\cdot \mid \widetilde{\xi}_{l}\right) \widetilde{\xi}_{l} \in \mathcal{B}(\mathcal{H}) .
$$

Then $X_{0}$ is an Iwasawa regular element of $\mathfrak{s p}_{\mathfrak{I}}$ and the Iwasawa decomposition of $\mathfrak{s p}_{\mathfrak{I}}$ associated with $X_{0}$ is

$$
\mathfrak{s p}_{\mathfrak{I}}=\left(\mathfrak{u}_{\mathfrak{I}} \cap \mathfrak{s p}_{\mathfrak{I}}\right) \dot{+}\left(\mathfrak{a}_{\mathfrak{I}, X_{0}} \cap \mathfrak{s p}_{\mathfrak{I}}\right) \dot{+}\left(\mathfrak{n}_{\mathfrak{I}, X_{0}} \cap \mathfrak{s p}_{\mathfrak{I}}\right)
$$

(where $\mathfrak{u}_{\mathfrak{I}}, \mathfrak{a}_{\mathfrak{I}, X_{0}}$, and $\mathfrak{n}_{\mathfrak{I}, X_{0}}$ are the ones defined in Proposition 4.3).

Moreover there exists a global Iwasawa decomposition $\boldsymbol{m}: K \times A \times N \rightarrow \mathrm{Sp}_{\mathfrak{I}}$ corresponding to (6.1). In addition we have $A N=N A$, and both groups $A$ and $N$ are simply connected.

Proof. One can proceed just as in the proof of Proposition [5.1, now using the orthogonal projection $\widetilde{P}_{r}: \mathcal{H} \rightarrow \mathcal{H}$ onto the subspace

$$
\widetilde{\mathcal{H}}_{r}=\operatorname{span}\left\{\widetilde{\xi}_{1}, \widetilde{\xi}_{-1}, \widetilde{\xi}_{2}, \widetilde{\xi}_{-2}, \ldots, \widetilde{\xi}_{r}, \widetilde{\xi}_{-r}\right\}
$$

for $r=1,2, \ldots$ We omit the details.

To prove the assertion on the global Iwasawa decomposition one can use Proposition 2.15] in a fashion similar to that of the proof of Theorem 4.6.

\section{Real groups of type CI.}

Theorem 6.2. Let $\widetilde{J}: \mathcal{H} \rightarrow \mathcal{H}$ be an anti-conjugation and $J: \mathcal{H} \rightarrow \mathcal{H}$ a conjugation such that $J \widetilde{J}=\widetilde{J} J$. Assume that $\left\{\widetilde{\xi}_{l}\right\}_{l \in \mathbb{Z} \backslash\{0\}}$ is an orthonormal basis in $\mathcal{H}$ such that $\widetilde{J} \widetilde{\xi}_{ \pm l}=\mp \widetilde{\xi}_{\mp l}$ and $J \widetilde{\xi}_{ \pm l}=\widetilde{\xi}_{ \pm l}$ for $l=1,2, \ldots$ Now pick a family of mutually different real numbers $\left\{\alpha_{l}\right\}_{l \in \mathbb{Z} \backslash\{0\}}$ satisfying the conditions

$\alpha_{-l}=-\alpha_{l}$ for all $l \in \mathbb{Z} \backslash\{0\}, \quad \lim _{\alpha \rightarrow \infty} \alpha_{l}=0, \quad$ and $\Phi\left(\alpha_{1}, \alpha_{-1}, \alpha_{2}, \alpha_{-2}, \ldots\right)<\infty$, and define the self-adjoint operator

$$
X_{0}=\sum_{l \in \mathbb{Z} \backslash\{0\}} \alpha_{l}\left(\cdot \mid \widetilde{\xi}_{l}\right) \widetilde{\xi}_{l} \in \mathcal{B}(\mathcal{H}) .
$$

Then $X_{0}$ is an Iwasawa regular element of $\mathfrak{s p}_{\mathfrak{I}}(\mathcal{H} ; \mathbb{R})$ and the corresponding Iwasawa decomposition is

$$
\mathfrak{s p}_{\mathfrak{I}}(\mathcal{H} ; \mathbb{R})=\left(\mathfrak{u}_{\mathfrak{I}} \cap \mathfrak{s p}_{\mathfrak{I}}(\mathcal{H} ; \mathbb{R})\right) \dot{+}\left(\mathfrak{a}_{\mathfrak{J}, X_{0}} \cap \mathfrak{s p}_{\mathfrak{I}}(\mathcal{H} ; \mathbb{R})\right) \dot{+}\left(\mathfrak{n}_{\mathfrak{I}, X_{0}} \cap \mathfrak{s p}_{\mathfrak{I}}(\mathcal{H} ; \mathbb{R})\right)
$$

(where $\mathfrak{u}_{\mathfrak{I}}, \mathfrak{a}_{\mathfrak{I}, X_{0}}$, and $\mathfrak{n}_{\mathfrak{I}, X_{0}}$ are the ones defined in Proposition 4.3).

Moreover, if $G$ stands for the connected 1-component of $\operatorname{Sp}_{\mathfrak{I}}(\mathcal{H} ; \mathbb{R})$, then there exists a global Iwasawa decomposition $\boldsymbol{m}: K \times A \times N \rightarrow G$ corresponding to (6.3). In addition we have $A N=N A$, and both groups $A$ and $N$ are simply connected.

Proof. The existence of an orthonormal basis as in the statement follows at once by Lemma 3.5 .

Let us prove that $X_{0} \in \mathfrak{s p}_{\mathfrak{I}}(\mathcal{H} ; \mathbb{R})=\mathfrak{s p}_{\mathfrak{I}} \cap \mathfrak{g l}_{\mathfrak{I}}(\mathcal{H} ; \mathbb{R})$. In fact $X_{0} \in \mathfrak{s p}_{\mathfrak{I}}$ by Proposition 6.1. On the other hand, for all $l \in \mathbb{Z} \backslash\{0\}$ and $\eta \in \mathcal{H}$ we have $J\left(\left(\eta \mid \widetilde{\xi}_{l}\right) \widetilde{\xi}_{l}\right)=\left(\widetilde{\xi}_{l} \mid \eta\right) J \widetilde{\xi}_{l}=\left(J \eta \mid J \widetilde{\xi}_{l}\right) \widetilde{\xi}_{l}=\left(J \eta \mid \widetilde{\xi}_{l}\right) \widetilde{\xi}_{l}$, whence $J X_{0}=X_{0} J$, and thus $J \in \mathfrak{g l}_{\mathfrak{I}}(\mathcal{H} ; \mathbb{R})$ as well. 
Moreover, just as in the proof of Theorem 4.7. it follows that $X_{0}$ is an Iwasawa quasi-regular element of $\mathfrak{s p}_{\mathfrak{I}}(\mathcal{H} ; \mathbb{R})$ and the corresponding Iwasawa decomposition looks as asserted. Finally, since $X_{0}$ is Iwasawa regular in $\mathfrak{s p}_{\mathfrak{I}}$ by Theorem 6.1] it follows that it is Iwasawa regular in $\mathfrak{s p}_{\mathfrak{I}}(\mathcal{H} ; \mathbb{R})$ as well.

To prove the assertion on the global Iwasawa decomposition one can use Proposition 2.15 in a fashion similar to that of the proof of Theorem 4.6 .

\section{Real groups of type CII.}

Theorem 6.3. Assume that we have an orthogonal direct sum decomposition $\mathcal{H}=$ $\mathcal{H}_{+} \oplus \mathcal{H}_{-}$with $\operatorname{dim} \mathcal{H}_{+}=\operatorname{dim} \mathcal{H}_{-}$, and let $\widetilde{J}: \mathcal{H} \rightarrow \mathcal{H}$ be an anti-conjugation such that $\widetilde{J}\left(\mathcal{H}_{ \pm}\right) \subseteq \mathcal{H}_{ \pm}$. Also let $V=\left(\begin{array}{cc}1 & 0 \\ 0 & -1\end{array}\right)$ with respect to this orthogonal direct sum decomposition of $\mathcal{H}$. Now let $\bigcup_{l \in \mathbb{Z} \backslash\{0\}}\left\{e_{l}^{+}, e_{l}^{-}\right\}$be an orthonormal basis in $\mathcal{H}$ such that $\widetilde{J} e_{ \pm l}^{\varepsilon}=\mp e_{\mp l}^{\varepsilon}$ and $V e_{ \pm l}^{\varepsilon}=\varepsilon e_{ \pm l}^{\varepsilon}$ whenever $\varepsilon \in\{+,-\}$ and $l=1,2, \ldots$ Then define $f_{l}^{ \pm}=\left(e_{l}^{+} \pm e_{l}^{-}\right) / \sqrt{2}$ for all $l \in \mathbb{Z} \backslash\{0\}$. Pick a family of mutually different real numbers $\left\{\lambda_{l}\right\}_{l \in \mathbb{Z} \backslash\{0\}}$ such that

$\lambda_{-l}=-\lambda_{l}$ whenever $l \in \mathbb{Z} \backslash\{0\}, \lim _{l \rightarrow \infty} \lambda_{l}=0$, and $\Phi\left(\lambda_{1},-\lambda_{1}, \lambda_{2},-\lambda_{2}, \ldots\right)<\infty$, and define the self-adjoint operator

$$
X_{0}:=\sum_{l \in \mathbb{Z} \backslash\{0\}} \lambda_{l}\left(\left(\cdot \mid f_{l}^{+}\right) f_{l}^{+}-\left(\cdot \mid f_{l}^{-}\right) f_{l}^{-}\right) .
$$

Then $X_{0}$ is an Iwasawa regular element of $\mathfrak{s p}_{\mathfrak{I}}\left(\mathcal{H}_{+}, \mathcal{H}_{-}\right)$and the corresponding Iwasawa decomposition is

$$
\begin{aligned}
\mathfrak{s p}_{\mathfrak{I}}\left(\mathcal{H}_{+}, \mathcal{H}_{-}\right)= & \left(\mathfrak{u}_{\mathfrak{I}} \cap \mathfrak{s p}_{\mathfrak{I}}\left(\mathcal{H}_{+}, \mathcal{H}_{-}\right)\right) \dot{+}\left(\mathfrak{a}_{\mathfrak{I}, X_{0}} \cap \mathfrak{s p}_{\mathfrak{I}}\left(\mathcal{H}_{+}, \mathcal{H}_{-}\right)\right) \\
& \dot{+}\left(\mathfrak{n}_{\mathfrak{I}, X_{0}} \cap \mathfrak{s p}_{\mathfrak{I}}\left(\mathcal{H}_{+}, \mathcal{H}_{-}\right)\right)
\end{aligned}
$$

(where $\mathfrak{u}_{\mathfrak{J}}, \mathfrak{a}_{\mathfrak{I}, X_{0}}$, and $\mathfrak{n}_{\mathfrak{I}, X_{0}}$ are the ones defined in Proposition 4.3).

Moreover, if $G$ stands for the connected 1 -component of $\operatorname{Sp}_{\mathfrak{I}}\left(\mathcal{H}_{+}, \mathcal{H}_{-}\right)$, then there exists a global Iwasawa decomposition $m: K \times A \times N \rightarrow G$ corresponding to (6.2). In addition we have $A N=N A$, and both groups $A$ and $N$ are simply connected.

Proof. The existence of the orthonormal basis

$$
\bigcup_{l \in \mathbb{Z} \backslash\{0\}}\left\{e_{l}^{+}, e_{l}^{-}\right\}
$$

follows by Lemma 3.5. Just as in the proof of Proposition 4.8 we can see that $X_{0} \in \mathfrak{u}_{\mathfrak{I}}\left(\mathcal{H}_{+}, \mathcal{H}_{-}\right)$. On the other hand, Lemma 3.8(b) shows that $X_{0} \in \mathfrak{s p}_{\mathfrak{I}}$, and thus $X_{0} \in \mathfrak{s p}_{\mathfrak{I}} \cap \mathfrak{u}_{\mathfrak{I}}\left(\mathcal{H}_{+}, \mathcal{H}_{-}\right)=\mathfrak{s p}_{\mathfrak{I}}\left(\mathcal{H}_{+}, \mathcal{H}_{-}\right)$. Then, by using Corollary 2.13 along with the orthogonal projections on the subspaces

$$
\mathcal{H}_{r}=\operatorname{span}\left(\bigcup_{-r \leq l \leq r}\left\{e_{l}^{+}, e_{l}^{-}\right\}\right) \text {for } r=1,2, \ldots,
$$

one can prove that $X_{0}$ is an Iwasawa quasi-regular element of $\mathfrak{s p}_{\mathfrak{I}}\left(\mathcal{H}_{+}, \mathcal{H}_{-}\right)$and the corresponding Iwasawa decomposition looks as asserted. (See the proof of Proposition 4.8 for more details.)

Now it remains to show that $X_{0}$ is actually an Iwasawa regular element of $\mathfrak{s p}_{\mathfrak{I}}\left(\mathcal{H}_{+}, \mathcal{H}_{-}\right)$. To this end denote $\mathcal{V}_{j}^{0}=\mathbb{C} f_{j}^{+}+\mathbb{C} f_{-j}^{-}, \mathcal{V}_{j}^{1}=\mathbb{C} f_{j}^{-}+\mathbb{C} f_{-j}^{+}$, and 
$\mathcal{V}_{j}=\mathcal{V}_{j}^{0} \oplus \mathcal{V}_{j}^{1}$ for $j \geq 1$. Then let $X=X^{*} \in \mathfrak{s p}_{\mathfrak{I}}\left(\mathcal{H}_{+}, \mathcal{H}_{-}\right)$such that $\left[X, X_{0}\right]=0$. We have $\lambda_{-l}=-\lambda_{l}$ whenever $l \in \mathbb{Z} \backslash\{0\}$; hence

$$
X_{0}:=\sum_{j \geq 1} \lambda_{j}\left(\left(\cdot \mid f_{j}^{+}\right) f_{j}^{+}+\left(\cdot \mid f_{-j}^{-}\right) f_{-j}^{-}\right)-\lambda_{j}\left(\left(\cdot \mid f_{j}^{-}\right) f_{j}^{-}+\left(\cdot \mid f_{-j}^{+}\right) f_{-j}^{+}\right) .
$$

Since the real numbers $\left\{\lambda_{j}\right\}_{j \geq 1}$ are mutually different and $\left[X, X_{0}\right]=0$, it follows that $X$ leaves both the subspaces $\mathcal{V}_{j}^{0}$ and $\mathcal{V}_{j}^{1}$ invariant whenever $j=1,2, \ldots$ Now let us keep $j \in\{1,2, \ldots\}$ and $\varepsilon \in\{0,1\}$ fixed. Since $X=X^{*}$, there exist $x_{0} \in \mathcal{V}_{j}^{\varepsilon} \backslash\{0\}$ and $t_{0} \in \mathbb{R}$ such that $X x_{0}=t_{0} x_{0}$. On the other hand, since $V \widetilde{J}=\widetilde{J} V$, it follows directly that $\widetilde{J}_{1}:=V \widetilde{J}$ is an anti-conjugation on $\mathcal{H}$. In addition, since $V f_{ \pm j}^{\varepsilon}=f_{ \pm j}^{-\varepsilon}$ and $\widetilde{J} f_{ \pm j}^{\varepsilon}=\mp f_{\mp j}^{\varepsilon}$, it follows that the linear subspace $\mathcal{V}_{j}^{\varepsilon}$ is invariant under the anti-conjugation $\widetilde{J}_{1}$. Let us endow $\mathcal{V}_{j}^{\varepsilon}$ with the corresponding quaternionic structure. Since $\operatorname{dim}_{\mathbb{C}} \mathcal{V}_{j}^{\varepsilon}=2$, it follows that $\operatorname{dim}_{\mathbb{H}} \mathcal{V}_{j}^{\varepsilon}=1$, and thus $\mathcal{V}_{j}^{\varepsilon}=\mathbb{H} x_{0}$. On the other hand $X=X^{*} \in \mathfrak{s p}_{\mathfrak{I}}\left(\mathcal{H}_{+}, \mathcal{H}_{-}\right)$; hence $X V=-V X$ and $X \widetilde{J}=-\widetilde{J} X$, whence $X \widetilde{J}_{1}=\widetilde{J}_{1} X$. Thus $X$ is an $\mathbb{H}$-linear operator with respect to the quaternionic structure defined by the anti-conjugation $\widetilde{J}_{1}$. Now, since $\mathcal{V}_{j}^{\varepsilon}=\mathbb{H} x_{0}$ and $X x_{0}=t_{0} x_{0}$, it follows that the restriction of $X$ to $\mathcal{V}_{j}^{\varepsilon}$ is given by the multiplication by the real number $t_{0}$. Since $\mathcal{H}=\bigoplus_{j \geq 1}\left(\mathcal{V}_{j}^{0} \oplus \mathcal{V}_{j}^{1}\right)$, it thus follows that the operators in $\mathfrak{a}_{\mathfrak{I}, X_{0}} \cap \mathfrak{s p}_{\mathfrak{I}}\left(\mathcal{H}_{+}, \mathcal{H}_{-}\right)$commute pairwise, and this completes the proof.

To prove the assertion on the global Iwasawa decomposition one can use Proposition 2.15 in a fashion similar to that of the proof of Theorem 4.6 .

\section{DeCompositions Lifted TO COVERING GROUPS}

The aim of this short section is to show that the Iwasawa decompositions constructed in Sections 4 , 5, and 6 can be lifted to any covering groups. We refer to Ha72] and [Nee02a] for information on the homotopy groups of the classical Banach-Lie groups associated with the Schatten ideals. It is easy to see that the corresponding description of homotopy groups actually holds true for the classical Banach-Lie groups associated with any separable norm ideal.

Proposition 7.1. Let $G$ be a connected Banach-Lie group, and let $K, A$, and $N$ be connected Banach-Lie subgroups of $G$ such that the multiplication map

$$
\boldsymbol{m}: K \times A \times N \rightarrow G
$$

is a diffeomorphism. In addition, assume that $A$ and $N$ are simply connected and $A N=N A$.

Now assume that we have a connected Banach-Lie group $\widetilde{G}$ with a covering homomorphism $e: \widetilde{G} \rightarrow G$, and define $\widetilde{K}:=e^{-1}(K), \widetilde{A}:=e^{-1}(A)$, and $\widetilde{N}:=e^{-1}(N)$. Then $\widetilde{K}, \widetilde{A}$, and $\widetilde{N}$ are connected Banach-Lie subgroups of $\widetilde{G}$ and the multiplication map $\widetilde{\boldsymbol{m}}: \widetilde{K} \times \widetilde{A} \times \widetilde{N} \rightarrow \widetilde{G}$ is a diffeomorphism.

Proof. The proof can be achieved by using appropriate infinite-dimensional versions of some standard ideas from the theory of Iwasawa decompositions of reductive groups (specifically, see for instance the proofs of Theorems 6.31 and 6.46 in [Kn96]). We refer to Proposition 4.4 in $\mathrm{Be} 09$ for details. 
Corollary 7.2. Let $\mathcal{H}$ be a complex separable infinite-dimensional Hilbert space, $\Phi$ a mononormalizing symmetric norming function whose Boyd indices are nontrivial, and denote the corresponding separable norm ideal by $\mathfrak{I}=\mathfrak{S}_{\Phi}^{(0)} \subseteq \mathcal{B}(\mathcal{H})$. Then let $\boldsymbol{m}: K \times A \times N \rightarrow G$ be the global Iwasawa decomposition given by any of Theorems 4.5, 4.6, 4.7, 4.8, 5.2, 5.3, 6.1, 6.2 and 6.3 for the connected 1-components of real or complex classical Banach-Lie groups. Now denote by $e: \widehat{G} \rightarrow G$ any covering group of $G$. If we define $\widehat{K}:=e^{-1}(K), \widehat{A}:=e^{-1}(A)$, and $\widehat{N}:=e^{-1}(N)$, then $\widehat{K}, \widehat{A}$, and $\widehat{N}$ are connected Banach-Lie subgroups of $\widetilde{G}$ and the multiplication map $\widehat{\boldsymbol{m}}: \widehat{K} \times \widehat{A} \times \widehat{N} \rightarrow \widehat{G}$ is a diffeomorphism.

Proof. Use Proposition 7.1 .

\section{Appendix A. Auxiliary faCts on Operator ideals}

In this appendix we record some facts on operator ideals, stating them under versions appropriate for use in the main body of the present paper. We refer to GK69, GK70, Er72, EL72, Er78, KW02, We05, [DFWW], KW06, Be06, and $[\mathrm{Be} 09]$ for various special topics involving symmetric norm ideals related to the circle of ideas discussed here.

Let $\mathcal{H}$ be a complex Hilbert space and $\mathfrak{P}$ a maximal nest in $\mathcal{B}(\mathcal{H})$. That is, $\mathfrak{P}$ is a maximal linearly ordered set of orthogonal projections on $\mathcal{H}$. Then we denote $\operatorname{Alg} \mathfrak{P}:=\{b \in \mathcal{B}(\mathcal{H}) \mid(\forall p \in \mathfrak{P}) \quad b p=p b p\}$ (the nest algebra associated with $\mathfrak{P}$ ).

In the following statement we need the notion of Boyd indices as used in Ara78. (see also subsections 2.17-2.19 in [DFWW]).

Theorem A.1. Assume that $\mathcal{H}$ is a complex separable Hilbert space and $\mathfrak{P}$ is a maximal nest in $\mathcal{B}(\mathcal{H})$. Let $\Phi$ be a mononormalizing symmetric norming function whose Boyd indices are nontrivial and denote $\mathfrak{I}=\mathfrak{S}_{\Phi}^{(0)}$. Then for every $a \in \mathrm{GL}_{\mathfrak{I}}(\mathcal{H})$ such that $0 \leq a$ there exist uniquely determined operators $d \in \mathrm{GL}_{\mathfrak{I}}(\mathcal{H})$ and $r \in \mathfrak{I}$ satisfying the following conditions:

- $0 \leq d \in \mathrm{GL}_{\mathfrak{I}}(\mathcal{H}) \cap \operatorname{Alg} \mathfrak{P}$;

- $r \in \mathfrak{I} \cap \operatorname{Alg} \mathfrak{P}$ and the spectrum of $r$ is equal to $\{0\}$;

- $a=\left(\mathbf{1}+r^{*}\right) d(\mathbf{1}+r)$.

Proof. Theorem 4.1 in Ara78] and Lemma 4.3 in [EL72] show that Theorem 4.2 in [Er72] (or Theorem 6.2 in Chapter IV of [GK70]) applies for the operator ideals $\mathfrak{S}_{\mathrm{I}}=\mathfrak{S}_{\mathrm{II}}=\mathfrak{S}_{\Phi}^{(0)}$.

Corollary A.2. Let $\mathfrak{P}, \Phi$, and $\mathfrak{I}$ be as in Theorem A.1. Then for every operator $g \in \mathrm{GL}_{\mathfrak{I}}(\mathcal{H})$ there exist $b \in \mathrm{GL}_{\mathfrak{I}}(\mathcal{H}) \cap \operatorname{Alg} \mathfrak{P}$ and $u \in \mathrm{U}_{\mathfrak{I}}(\mathcal{H})$ such that $g=u b$.

Proof. By applying Theorem A.1 for $a=g^{*} g$ we get the operators $d \in \mathrm{GL}_{\mathfrak{I}}(\mathcal{H})$ and $r \in \mathfrak{I}$ such that $g^{*} g=\left(\mathbf{1}+r^{*}\right) d(\mathbf{1}+r)$. Now denote $c=\mathbf{1}+r \in \mathrm{GL}_{\mathfrak{I}}(\mathcal{H}) \cap \operatorname{Alg} \mathfrak{P}$. Then $g^{*} g=c^{*} d c, d \geq 0$, and all of the operators $g, c$, and $d$ are invertible; hence the operator $u:=g\left(d^{1 / 2} c\right)^{-1}$ is unitary. On the other hand, since $0 \leq d \in \operatorname{GL}_{\mathfrak{I}}(\mathcal{H})$, it is straightforward to prove that $d^{1 / 2} \in \mathrm{GL}_{\mathfrak{I}}(\mathcal{H})$, whence $u \in \mathrm{U}_{\mathfrak{I}}(\mathcal{H})$.

In addition we have $b:=d^{1 / 2} c \in \mathrm{GL}_{\mathfrak{I}}(\mathcal{H}) \cap \operatorname{Alg} \mathfrak{P}$ and $g=u b$, and this completes the proof.

Example A.3. Theorem A.1 and Corollary A.2 apply in particular for the Schatten ideal $\mathfrak{I}=\mathfrak{S}_{p}(\mathcal{H})$ if $1<p<\infty$. 


\section{ACKNOWLEDGMENT}

The author thanks Professor José Galé, Professor Karl-Hermann Neeb, and Professor Tudor Ratiu for encouragement, and Professor Hendrik Grundling and Professor Gary Weiss for kindly pointing out some relevant references.

This work was partially supported by Grant 2-CEx06-11-34/25.07.2006 of the Romanian Government.

\section{REFERENCES}

[Ara78] J. Arazy, Some remarks on interpolation theorems and the boundness of the triangular projection in unitary matrix spaces, Integral Equations Operator Theory 1 (1978), no. 4, 453-495. MR.516764 (81k:47056a)

[Arv67] W.B. Arveson, Analyticity in operator algebras, Amer. J. Math. 89 (1967), 578-642. MR0223899 (36:6946)

[Arv75] W.B. Arveson, Interpolation problems in nest algebras, J. Functional Analysis 20 (1975), no. 3, 208-233. MR0383098 (52:3979)

[Ba69] V.K. Balachandran, Simple $L^{*}$-algebras of classical type, Math. Ann. 180 (1969), 205219. MR 0243362 (39:4684)

[BD01] V. Bălan, J. Dorfmeister, Birkhoff decompositions and Iwasawa decompositions for loop groups, Tohoku Math. J. 53 (2001), 593-615. MR.1862221 (2002j:22023)

[Be05] D. Beltiţă, On Banach-Lie algebras, spectral decompositions and complex polarizations. In: D. Gaşpar, I. Gohberg, D. Timotin, F.-H. Vasilescu, L. Zsido (eds.), Recent Advances in Operator Theory, Operator Algebras, and Their Applications. XIXth International Conference on Operator Theory, Timisoara (Romania), 2002. Operator Theory: Advances and Applications, 153. Birkhäuser Verlag, Basel, 2005, pp. 13-38. MR2105467 (2005k:58009)

[Be06] D. Beltiţă, Smooth Homogeneous Structures in Operator Theory, Monographs and Surveys in Pure and Applied Mathematics, 137. Chapman \& Hall/CRC Press, Boca RatonLondon-New York-Singapore, 2006. MR2188389 (2007c:58010)

[Be09] D. Beltiţă, Functional analytic background for a theory of infinite-dimensional reductive Lie groups, in: K.-H. Neeb, A. Pianzola (eds.), Developments and Trends in Infinite Dimensional Lie Theory, Progress in Mathematics, Birkhäuser Verlag, Basel 2009.

[BP07] D. Beltiţă, B. Prunaru, Amenability, completely bounded projections, dynamical systems and smooth orbits, Integral Equations Operator Theory 57 (2007), no. 1, 1-17. MR.2294192 (2008d:46076)

[BR05] D. Beltiţă, T.S. Ratiu, Symplectic leaves in real Banach Lie-Poisson spaces, Geom. Funct. Anal. 15 (2005), no. 4, 753-779. MR2221149 (2007k:58008)

[BR07] D. Beltiţă, T.S. Ratiu, Geometric representation theory for unitary groups of operator algebras, Adv. Math. 208 (2007), no. 1, 299-317. MR2304319

[BS01] D. Beltiţă, M. Şabac, Lie Algebras of Bounded Operators, Operator Theory: Advances and Applications, 120. Birkhäuser Verlag, Basel, 2001. MR.1825892 (2003b:47107)

[BFR93] A.M. Bloch, H. Flaschka, T.S. Ratiu, A Schur-Horn-Kostant convexity theorem for the diffeomorphism group of the annulus, Invent. Math. 113 (1993), no. 3, 511-529. MR.1231835 (94i:58063)

[Bo80] R.P. Boyer, Representation theory of the Hilbert-Lie group $\mathrm{U}(\mathfrak{H})_{2}$, Duke Math. J. 47 (1980), no. 2, 325-344. MR575900 (81g:22024)

[Bo93] R.P. Boyer, Representation theory of infinite-dimensional unitary groups, in: Representation theory of groups and algebras, Contemp. Math., 145, Amer. Math. Soc., Providence, RI, 1993, pp. 381-391. MR1216198 (94g:22038)

[Ca85] A.L. Carey, Some homogeneous spaces and representations of the Hilbert Lie group $\mathcal{U}(H)_{2}$, Rev. Roumaine Math. Pures Appl. 30 (1985), no. 7, 505-520. MR826232 (87e:22044)

[CG99] G. Corach, J.E. Galé, On amenability and geometry of spaces of bounded representations, J. London Math. Soc. (2) 59 (1999), no. 1, 311-329. MR1688504 (2000f:46064)

[Da88] K.R. Davidson, Nest Algebras, Pitman Research Notes in Mathematics Series, 191. Longman Scientific \& Technical, Harlow; copublished in the United States with John Wiley \& Sons, Inc., New York, 1988. MR.972978 (90f:47062) 
[DPW02] I. Dimitrov, I. Penkov, J.A. Wolf, A Bott-Borel-Weil theory for direct limits of algebraic groups, Amer. J. Math. 124 (2002), no. 5, 955-998. MR1925340 (2003k:20064)

[DFWW] K. Dykema, T. Figiel, G. Weiss, M. Wodzicki, Commutator structure of operator ideals, Adv. Math. 185(2004), no. 1, 1-79. MR2058779 (2005f:47149)

[Er72] J.A. Erdos, The triangular factorization of operators on Hilbert space, Indiana Univ. Math. J. 22 (1972/73), 939-950. MR0336404(49:1179)

[Er78] J.A. Erdos, Triangular integration on symmetrically normed ideals, Indiana Univ. Math. J. 27 (1978), no. 3, 401-408. MR0473892 (57:13551)

[EL72] J.A. Erdos, W.E. Longstaff, The convergence of triangular integrals of operators on Hilbert space, Indiana Univ. Math. J. 22 (1972/73), 929-938. MR0336403 (49:1178)

[Ga06] J.E. Galé, Geometría de órbitas de representaciones de grupos y álgebras promediables, Rev. R. Acad. Cienc. Exactas Fís. Quím. Nat. Zaragoza (2) 61 (2006), 7-46. MR 2311998

[GK69] I.C. Gohberg, M.G. Kreĭn, Introduction to the Theory of Linear Nonselfadjoint Operators, Translations of Mathematical Monographs, vol. 18, American Mathematical Society, Providence, RI, 1969. MR.0246142 (39:7447)

[GK70] I.C. Gohberg, M.G. Kreı̆n, Theory and Applications of Volterra Operators in Hilbert Space. Translated from the Russian by A. Feinstein. Translations of Mathematical Monographs, Vol. 24, American Mathematical Society, Providence, RI, 1970. MR 0264447 (41:9041)

[Gru05] H. Grundling, Generalising group algebras, J. London Math. Soc. (2) 72 (2005), no. 3, 742-762. MR2190335 (2006i:46079)

[Ha72] P. de la Harpe, Classical Banach-Lie Algebras and Banach-Lie Groups of Operators in Hilbert Space, Lecture Notes in Mathematics 285, Springer-Verlag, Berlin-HeidelbergNew York, 1972. MR0476820(57:16372)

[HK77] L.A. Harris, W. Kaup, Linear algebraic groups in infinite dimensions, Illinois J. Math. 21(1977), 666-674. MR0460551 (57:544)

[He01] S. Helgason, Differential Geometry, Lie Groups, and Symmetric Spaces. (Corrected reprint of the 1978 original). Graduate Studies in Mathematics, 34. American Mathematical Society, Providence, RI, 2001. MR1834454 (2002b:53081)

[Iw49] K. Iwasawa, On some types of topological groups, Ann. of Math. (2) 50 (1949), 507558. MR0029911(10:679a)

[KW02] V. Kaftal, G. Weiss, Traces, ideals, and arithmetic means, Proc. Natl. Acad. Sci. USA 99 (2002), no. 11, 7356-7360. MR1907839 (2003e:47119)

[KW06] V. Kaftal, G. Weiss, $B(H)$ lattices, density and arithmetic mean ideals, preprint, 2006.

[Ke04] P. Kellersch, Eine Verallgemeinerung der Iwasawa Zerlegung in Loop Gruppen (Dissertation, TU-München, 1999), Differential Geometry - Dynamical Systems. Monogr. 4. Geometry Balkan Press, Bucharest, 2004. MR2158164 (2006b:22017)

[Ki73] A.A. Kirillov, Representations of the infinite-dimensional unitary group. (Russian) Dokl. Akad. Nauk. SSSR 212 (1973), 288-290. MR0340487 (49:5239)

[Kn96] A.W. Knapp, Lie Groups Beyond an Introduction, Progress in Mathematics 140, Birkhäuser-Verlag, Boston-Basel-Berlin, 1996. MR.1399083 (98b:22002)

[Ko73] B. Kostant, On convexity, the Weyl group and the Iwasawa decomposition, Ann. Sci. École Norm. Sup. (4) 6 (1973), 413-455. MR0364552 (51:806)

[Lan01] S. Lang, Fundamentals of Differential Geometry (corrected second printing), Graduate Texts in Mathematics, 191. Springer-Verlag, New York, 2001. MR.1666820 (99m:53001)

[Lar85] D.R. Larson, Nest algebras and similarity transformations, Ann. of Math. (2) 121 (1985), no. 3, 409-427. MR794368 (86j:47061)

[LR91] J.-H. Lu, T.S. Ratiu, On the nonlinear convexity theorem of Kostant, J. Amer. Math. Soc. 4 (1991), no. 2, 349-363. MR1086967 (92a:58048)

[MSS88] P.S. Muhly, K.-S. Saito, B. Solel, Coordinates for triangular operator algebras, Ann. of Math. (2) 127 (1988), no. 2, 245-278. MR.932297 (89h:46088)

[NRW01] L. Natarajan, E. Rodríguez-Carrington, J.A. Wolf, The Bott-Borel-Weil theorem for direct limit groups, Trans. Amer. Math. Soc. 353 (2001), no. 11, 4583-4622. MR.1650034 (2002a:22013)

[Nee98] K.-H. Neeb, Holomorphic highest weight representations of infinite-dimensional complex classical groups, J. reine angew. Math. 497 (1998), 171-222. MR.1617431 (99h:22027) 
[Nee02a] K.-H. Neeb, Classical Hilbert-Lie groups, their extensions and their homotopy groups, in: A. Strasburger, J. Hilgert, K.-H. Neeb, W. Wojtyński (eds.), Geometry and Analysis on Finite and Infinite-dimensional Lie Groups (Będtewo, 2000), Banach Center Publ., vol. 55, Polish Acad. Sci. Warsaw, 2002, pp. 87-151. MR1911982 (2003e:58007)

[Nee02b] K.-H. Neeb, A Cartan-Hadamard theorem for Banach-Finsler manifolds, Geom. Dedicata 95 (2002), 115-156. MR 1950888 (2004a:58004)

[Nee04] K.-H. Neeb, Infinite-dimensional groups and their representations, in: Lie Theory, Progr. Math. 228, Birkhäuser, Boston, MA, 2004, pp. 213-328. MR2042690 (2005f:22031)

[NØ98] K.-H. Neeb, B. Ørsted, Unitary highest weight representations in Hilbert spaces of holomorphic functions on infinite-dimensional domains, J. Funct. Anal. 156 (1998), no. 1, 263-300. MR1632917 (99m:22022)

[Neu99] A. Neumann, An infinite-dimensional version of the Schur-Horn convexity theorem, $J$. Funct. Anal. 161 (1999), no. 2, 418-451. MR.1674643 (2000a:22030)

[Neu02] A. Neumann, An infinite dimensional version of the Kostant convexity theorem, $J$. Funct. Anal. 189 (2002), no. 1, 80-131. MR.1887630 (2003d:47100)

[O178] G.I. Ol'šanskiı̌, Unitary representations of the infinite-dimensional classical groups $\mathrm{U}(p, \infty), \mathrm{SO}_{0}(p, \infty), \mathrm{Sp}(p, \infty)$, and of the corresponding motion groups. (Russian) Funktsional. Anal. i Prilozhen. 12 (1978), no. 3, 32-44, 96. MR509382 (80g:22020)

[Ol88] G.I. Ol'šanskiu, The method of holomorphic extensions in the theory of unitary representations of infinite-dimensional classical groups. (Russian) Funktsional. Anal. $i$ Prilozhen. 22 (1988), no. 4, 23-37, 96. MR976993 (90f:22025)

[Pa88] A.L.T. Paterson, Amenability, Mathematical Surveys and Monographs, 29. American Mathematical Society, Providence, RI, 1988. MR961261 (90e:43001)

[Pic90] D. Pickrell, Separable representations for automorphism groups of infinite symmetric spaces, J. Funct. Anal. 90 (1990), no. 1, 1-26. MR.1047575 (91h:22038)

[Pit88] D.R. Pitts, Factorization problems for nests: Factorization methods and characterizations of the universal factorization property, J. Funct. Anal. 79 (1988), no. 1, 57-90. MR.950084 (90a:46160)

[Po86] S.C. Power, Factorization in analytic operator algebras, J. Funct. Anal. 67 (1986), no. 3, 413-432. MR845465 (87k:47040)

[Se57] I.E. Segal, The structure of a class of representations of the unitary group on a Hilbert space, Proc. Amer. Math. Soc. 8 (1957), 197-203. MR0084122(18:812f)

[SV75] S. Strătilă, D. Voiculescu, Representations of AF-algebras and of the group $U(\infty)$, Lecture Notes in Mathematics, Vol. 486. Springer-Verlag, Berlin-New York, 1975. MR.0458188 (56:16391)

[Tu05] A.B. Tumpach, Variétés Kählériennes et Hyperkählériennes de Dimension Infinie, Ph.D. Thesis, École Polytechnique, Paris, 2005.

[Tu06] A.B. Tumpach, Mostow decomposition theorems for $L^{*}$-groups and applications to affine coadjoint orbits and stable manifolds, preprint math-ph/0605039.

[Up85] H. Upmeier, Symmetric Banach Manifolds and Jordan $C^{*}$-Algebras, North-Holland Math. Stud. 104, Notas de Matemática 96, North-Holland, Amsterdam, 1985. MR.776786 (87a:58022)

[We05] G. Weiss, $B(H)$-commutators: A historical survey. In: D. Gaşpar, I. Gohberg, D. Timotin, F.-H. Vasilescu, L. Zsido (eds.), Recent Advances in Operator Theory, Operator Algebras, and Their Applications. XIXth International Conference on Operator Theory, Timisoara (Romania), 2002. Operator Theory: Advances and Applications, 153. Birkhäuser Verlag, Basel, 2005, pp. 307-320. MR2105485 (2005j:47039)

[Wo05] J.A. Wolf, Principal series representations of direct limit groups, Compos. Math. 141 (2005), no. 6, 1504-1530. MR2188447 (2007d:22010)

Institute of Mathematics "Simion Stollow" of the Romanian Academy, P.O. Box 1764, Bucharest, Romania

E-mail address: Daniel.Beltita@imar.ro 